\title{
A Zinc Oxide Nanowire Pressure Sensor
}

by

Thomas Stanley van den Heever

Thesis presented in partial fulfilment of the requirements for the degree

Master of Science in Engineering at the University of Stellenbosch

Supervisor: Prof. Willem Jacobus Perold

Department of Electrical and Electronic Engineering

December 2010 


\section{Declaration}

By submitting this thesis electronically, I declare that the entirety of the work contained therein is my own, original work, and that I have not previously in its entirety or in part submitted it for obtaining any qualification.

December 2010

Copyright (C) 2010 University of Stellenbosch

All rights reserved 


\section{Abstract}

Measurement of pressure with zinc oxide $(\mathrm{ZnO})$ nanowires was investigated. $\mathrm{ZnO}$ exhibits the piezoelectric effect, generating a voltage when pressure is applied to the material. This relationship between pressure and output voltage was used to make a pressure sensor. A study of the physical and mathematical working of the piezoelectric effect in $\mathrm{ZnO}$ nanowires was done. Simulations were conducted by means of specialised software to test the theory. The simulations gave results as the theory had predicted. $\mathrm{ZnO}$ nanowires were grown using various methods. Vapour liquid solid (VLS) was found to be the best method to grow uniform and dense arrays of $\mathrm{ZnO}$ nanowires. Statistical methods were employed to obtain the optimal parameters for the growth of $\mathrm{ZnO}$ nanowires through the VLS method. After the growth of the $\mathrm{ZnO}$ nanowires a pressure sensor was built. The manufacturing of the pressure sensor consisted of different steps. The sensors were tested to verify that they worked as described in theory and as shown in the simulations. The output voltage was lower than the simulated value due to imperfections and losses throughout the system. The output voltage versus applied pressure graphs did coincide with the bulk $\mathrm{ZnO}$ materials as well as related products, such as force sensing resistors. The output voltage is too low, but there are various methods by which the output voltage can be increased. These methods are discussed. The finished sensor can be used to continuously monitor pressure on a plane. 


\section{Opsomming}

Die meting van druk deur sink oksied $(\mathrm{ZnO})$ nanodrade was ondersoek. $\mathrm{ZnO}$ toon die piëzo-elektriese effek - spanning word gegenereer wanneer druk op die materiaal aangewend word. Hierdie verhouding tussen druk en uitsetspanning is gebruik om 'n druksensor te vervaardig. 'n Studie van die fisiese en wiskundige werking van die piëzo-elektriese effek in $\mathrm{ZnO}$ nanodrade is gedoen. Simulasies deur middel van gespesialiseerde sagteware is uitgevoer om die teorie te bevestig. Die simulasies het resultate getoon soos deur die teorie beskryf word. $\mathrm{ZnO}$ nanodrade is gegroei deur verskillende metodes. Verdamping vloeistof vastestof (VVV) is as die beste metode gevind om uniforme en digte skikkings van $\mathrm{ZnO}$ nanodrade te kry. Statistiese metodes is aangewend om die optimale parameters vir die groei van $\mathrm{ZnO}$ nanodrade deur middel van die VVV metode te kry. Na afloop van die groei van die $\mathrm{ZnO}$ nanodrade is ' $\mathrm{n}$ druksensor vervaardig. Die vervaardigingsproses het uit verskillende stappe bestaan, ten einde die bou van 'n werkende druksensor uit die $\mathrm{ZnO}$ nanodrade te realiseer. Die sensors is getoets om te bevestig dat dit werk, soos beskryf deur die teorie en gewys in die simulasies. Die uitsetspanning was laer as wat verwag was as gevolg van onvolmaakthede en verliese in die hele stelsel. Die uitsetspanning teenoor druk grafieke van die sensor het ooreengestem met die van die grootmaat materiale, asook verwante produkte soos druk sensitiewe weerstande. Die uitset spanning is baie laag en daar bestaan verskillende maniere waarop die uitsetspanning verhoog kan word. Hierdie metodes word bespreek. 


\section{Acknowledgements}

The author would like to thank the following people for their contribution towards this project.

- The financial assistance of the National Research Foundation (NRF) towards the research is hereby acknowledged. Opinions expressed and conclusions arrived at, are those of the author and are not necessarily to be attributed to the NRF.

- Prof. W.J. Perold, my supervisor, for helping with basic ideas and controlling the project. Without his guidance I would not have finished this project.

- Mr. U. Büttner for always helping with the instrumentation and laboratory equipment. He is full of ideas that helped me when things looked gloomy.

- Mr. G.S.H. Maritz for helping with LaTeX and for joining me for our daily Fritos and Coke. Thanks also for just being a great friend and listening to me when I moaned.

- Mr. H.E. Loubser for also being a friend and for the advice he gave me from time to time.

- To all my other friends, thanks.

- My family for all their support. They also listened to me when things were not going well. Thanks for all your love and comfort.

- To all the people in E211A: Thanks for a warm first year.

- To all the people in E210: Thanks for letting me join your office with aircon, and all the great times we had in the office. 
- Janine, my girlfriend, thanks for supporting me and loving me. 


\section{Contents}

$\begin{array}{lll}\text { Abstract } & \text { ii }\end{array}$

Opsomming

Acknowledgements iv

Contents $\quad$ vi

Abbreviations $\quad x$

List of Figures $\quad$ xii

List of Tables $\quad$ xix

1 Introduction 1

2 Nanotechnology 4

2.1 What is nanotechnology? . . . . . . . . . . . . . . 4

2.2 History of nanotechnology . . . . . . . . . . . . 5

2.3 Future of nanotechnology . . . . . . . . . . 7

3 Pressure Sensors $\quad 10$

3.1 What is Pressure? . . . . . . . . . . . . . . . . . 10

3.2 History of Pressure Measurements . . . . . . . . . . . . . 11

3.3 Different Pressure Measurement Equipment . . . . . . . . . 11

3.3.1 Sight Glass . . . . . . . . . . . . . . . . . . . . . . . 11

3.3.2 Magnetic Pressure Sensing . . . . . . . . . . . . . . . . 12

3.3.3 Capacitive Pressure Sensing . . . . . . . . . . . . . . 12

3.3.4 Piezoresistive Pressure Sensing . . . . . . . . . . . . . . . . . 13

3.3.5 Nano-sized Pressure Sensor . . . . . . . . . . . . . . . . 13 
4 Piezotronics $\quad 15$

4.1 What is Piezotronics? . . . . . . . . . . . . . . . . . 15

4.2 History of Piezotronics . . . . . . . . . . . . . . . 17

4.3 Nanopiezotronics . . . . . . . . . . . . . . . . . . . . . 18

4.3 .1 Field Effect Transistor _ . . . . . . . . . . . . . . 18

4.3 .2 Gated Diode . . . . . . . . . . . . . . . . . . . . . . . 19

4.4 Nanogenerator . . . . . . . . . . . . . . . . . 20

4.4.1 Nanogenerator basics . . . . . . . . . . . . . . 21

4.4.2 Mathematics of piezoelectric nanowire . . . . . . . 30

5 Nanostructures $\quad 37$

5.1 What are nanostructures? . . . . . . . . . . . . . 37

5.2 Crystal Structure . . . . . . . . . . . . . . . . . . . 38

5.3 Methods of growing nanostructures . . . . . . . . . . . 39

5.3.1 Vapour Liquid Solid . . . . . . . . . . . . . . . . 39

5.3.2 Laser Assisted Catalytic Growth . . . . . . . . . . . . . 45

5.3.3 Chemical Vapour Deposition . . . . . . . . . . . . 46

5.3.4 Template Assisted Synthesis . . . . . . . . . . . . . . . 49

5.3 .5 Electrospinning . . . . . . . . . . . . . 51

5.3 .6 Electrodeposition . . . . . . . . . . . . . . . . 53

5.4 Uses of nanostructures . . . . . . . . . . . . . . . . . . 56

6 Laboratory Equipment $\quad 57$

6.1 Scanning Tunneling Microscope . . . . . . . . . . . . . . . . 57

6.2 Atomic Force Microscope . . . . . . . . . . . . . . 58

6.3 Scanning Electron Microscope . . . . . . . . . . . . . . . . . . . 59

6.4 Microscopes . . . . . . . . . . . . . . . . . . . . 60

6.5 Furnace . . . . . . . . . . . . . . . . . . . . . . . . 60

6.6 Sputter Unit . . . . . . . . . . . . . . . . . . . . . . 62

6.7 Spin Table . . . . . . . . . . . . . . . . . . . . . . 63

6.8 Ultrasonic Mixer . . . . . . . . . . . . . . . . . . . . 64

6.9 Hotplate . . . . . . . . . . . . . . . . . . . . . . . 64

6.10 Sample Cutter . . . . . . . . . . . . . . . . . . 66

6.11 Scale . . . . . . . . . . . . . . . . . 67

6.12 Lithography . . . . . . . . . . . . . . . . 67

6.13 Mixer . . . . . . . . . . . . . . . . . . . . . 68

6.14 High-Voltage source . . . . . . . . . . . . . . . 68 
6.15 Thermal Evaporator . . . . . . . . . . . . . . . . . . 69

6.16 Sonic Mill . . . . . . . . . . . . . . . . . . 70

$\begin{array}{lll}7 & \text { Simulations } & 73\end{array}$

7.1 Introduction . . . . . . . . . . . . . 73

7.2 Comsol Simulations . . . . . . . . . . . . . . . . 74

8 Design of Experiments $\quad 83$

8.1 Theory ........................ 83

8.2 Design of experiment applied to the optimization of $\mathrm{ZnO}$ nanowire growth ....................... 85

9 Manufacturing of a $\mathrm{ZnO}$ nanowire pressure sensor 96

9.1 Introduction . . . . . . . . . . . . . . . . . . . 96

9.2 Manufacturing . . . . . . . . . . . . . . . 97

9.2 .1 Sample cutting . . . . . . . . . . . . . . 97

9.2 .2 Drilling . . . . . . . . . . . . . . . . . . . 99

9.2 .3 Pattern . . . . . . . . . . . . . . . . . . . 99

9.2.4 Growth of $\mathrm{ZnO}$ nanowires . . . . . . . . . . . . . . 99

9.2 .5 Gluing of two samples . . . . . . . . . . . . . . 102

9.2 .6 Wirebonding . . . . . . . . . . . . . . . . 103

9.3 Manufacturing difficulties . . . . . . . . . . . . . . . . . 103

9.3.1 Sample cutting . . . . . . . . . . . . . . . . 104

9.3 .2 Drilling . . . . . . . . . . . . . . . . . . . . . 104

9.3 .3 Pattern . . . . . . . . . . . . . . . . . . . 104

9.3.4 Growth of $\mathrm{ZnO}$ nanowires . . . . . . . . . . . . . . 104

9.3.5 Gluing of two samples . . . . . . . . . . . . . 105

9.3.6 Wirebonding . . . . . . . . . . . . . 105

9.4 Conclusion . . . . . . . . . . . . . . . . . . 105

10 Measurements and Results 107

10.1 Introduction . . . . . . . . . . . . . . . . . . . 107

10.2 Nanowire growth . . . . . . . . . . . . . . . . . . 107

10.3 Pressure versus Voltage ．. . . . . . . . . . . . . . . . . . . . . 109

10.4 Current Measurements . . . . . . . . . . . . . . . . . . . . . . 118

10.5 Conclusions . . . . . . . . . . . . . . . . . . . . . . . 119

$\begin{array}{ll}11 \text { Conclusions } & 121\end{array}$ 
CONTENTS

Bibliography

126

A Appendix A

135

B Appendix B 


\section{Abbreviations}

- AC - Alternating Current

- Ac - Acetone

- AFM - Atomic Force Microscope

- atm - atmospheric pressure

- $\mathrm{CB}$ - Conduction Band

- CNT - Carbon Nanotubes

- CPD - Cycles Per Degree

- CRN - Centre for Responsible Nanotechnology

- CVD - Chemical Vapor Deposition

- DC - Direct Current

- DoE - Design of Experiment

- DST - Department of Science and Technology

- EE - Effective Estimate

- $\mathrm{eV}$ - electron Volt

- FEM - Finite Element Method

- FET - Field Effect Transistor

- I - Current

- LED - Light Emiiting Diode 
- MEMS - Microelectromechanical Systems

- $\mu$ - micro $\left(10^{-6}\right)$

- MIT - Massachusetts Institute of Technology

- $\mathrm{nm}$ - nanometer $\left(10^{-9}\right)$ meter

- NNI - National Nanotechnology Institute

- NNS - National Nanotechnology Strategy

- $\mathrm{n}$ - nano $\left(10^{-9}\right)$

- Pa - Pascal

- SS - Sum of Squares

- STM - Scanning Tunneling Microscope

- UV - Ultra Violet

- V - Voltage

- VG - Voltage gap

- VLS - Vapor Liquid Solid 


\section{List of Figures}

2.1 IBM written with individual atoms [1] . . . . . . . . . 7

2.2 Timeline for nanotechnology $[2] \ldots \ldots \ldots \ldots$

3.1 Schematic of Magnetic Pressure Sensor [3] . . . . . . . . . . . . . . 12

3.2 Schematic of Capacitive Pressure Sensor [3] . . . . . . . . . . . 13

4.1 a) Crystal structure of $\mathrm{ZnO}$. b) Tetrahedral coordination between $\mathrm{Zn}$ and O. c) External force displaces the positive charge in relation to the negative charge, causing a potential difference [4]. . . 16

4.2 Schematic diagram of FET [5] . . . . . . . . . . . . . . . . . . . 19

4.3 Schematic diagram of nano-FET [4] . . . . . . . . . . . . . 19

4.4 Schematic diagram of nano-Gated Diode [5] . . . . . . . . . . 20

4.5 Different methods to harness electricity from the environment using a nanogenerator [6]. . . . . . . . . . . . . . . . 21

4.6 a) Visual representation of $\mathrm{ZnO}$ nanowire b) Strain induced in nanowire due to external force c) Electric field due to piezoelectric effect and d) Voltage induced due to the piezoelectric effect [4]. 23

4.7 a) Tip in contact with stretched side of nanowire b) Tip in contact with compressed side of nanowire [4]. . . . . . . . . . . .

4.8 a) The tip first comes into contact with the nanowire and forms a Schottky barrier b) The tip causes the nanowire to bend and in turn the nanowire generates a voltage, due to the piezoelectric effect and electrons begin to flow from the ground c) The tip reaches the middle of the nanowire and electrons start to flow to the ground and d) The tip reaches the other side of the nanowire and more electrons flow through the nanowire to ground [4]. . . . 
4.9 Two sets of nanowires brush against each other, thus creating a piezoelectric voltage. The top wires are coated with a metal to form a Schottky barrier between the bottom nanowires [7]. . . . . 26

4.10 Graphical representation of nanowires of different lengths [8]. . . 27

4.11 a) Nanowires of different length and their position relative to top electrode b) Circuit diagram of nanogenerator with nanowires in all possible configurations [9] . . . . . . . . . . . . 28

5.1 Crystal structure of $\mathrm{ZnO}[4] \ldots \ldots$

5.2 Wool-like $\mathrm{ZnO}$ structures [10] . . . . . . . . . . . . . . . . . 41

5.3 VLS growth visualized step by step [11]. . . . . . . . . . . . . 42

5.4 Picture of VLS growth as taken by SEM. Picture was taken with low magnification of only 1000. . . . . . . . . . . . . 44

5.5 Picture of VLS growth as taken by SEM. Picture was taken with high magnification. . . . . . . . . . . . . 45

5.6 (a) CVD vs (b) VLS growth. Images obtained via AFM . . . . . . . 49

5.7 Picture of a template used for nanowire growth [12] . . . . . . . 50

5.8 Electrospinning setup [13] . . . . . . . . . . . . . . . 51

5.9 SEM picture of $\mathrm{ZnO}$ nanowire growth with electrodeposition . . 55

6.1 Picture of Nanosurf easyScan 2.0 STM [14] . . . . . . . . . . . . 58

6.2 Picture of the internal operation of the AFM [15] . . . . . . . . . 59

6.3 Picture of Nanosurf easyScan 2.0 AFM [15]. . . . . . . . . . . . 60

6.4 Picture of the two microscope systems. The one on the left can magnify up to 30 times and the one on the right to 1.5 times. . . . 61

6.5 Screenshot of the software, Motic Images, used with the microscopes. . . . . . . . . . . . . . . . . 61

6.6 Picture of the tube furnace used for VLS growth. On the left are the gas bottles (out of sight) with the valve box just in picture. The furnace is the black object in the centre. Between the furnace and the valve box is the controller box. . . . . . . . . . . . . . 62

6.7 Software used with the furnace. The graph at the bottom shows temperature vs time. At the top are all the settings used to change the temperature and time. . . . . . . . . . . . . 63

6.8 Edwards Sputter Coater S150B . . . . . . . . . . . . . . 64 
6.9 Picture of the spin table. At the back, the silver area is the spinning area. The black box connected to the table is the control box, used to set the speed. On the floor, the black pedals are to start and stop the spinner. . . . . . . . . . . . . . . . . . . 65

6.10 Picture of the ultrasonic mixer. . . . . . . . . . . . . 65

6.11 Picture of the hotplate. On the left is the hotplate itself and on the right the controller box. . . . . . . . . . . . . 66

6.12 Picture of the sample cutter. . . . . . . . . . . . . . . . 66

6.13 Picture of the scale. . . . . . . . . . . . . . . . . . 67

6.14 Picture of the lithography system. . . . . . . . . . . . . . 68

6.15 Picture of the mixer. A flask with chemicals in it is placed on top and a small white magnet is placed inside. The dials are then turned to spin the magnet and heat up the flask. . . . . . . . . . .

6.16 Picture of the thermal evaporator. The sample is loaded into the glass dome with the source material. The system is then put under vacuum to achieve the thermal evaporation. . . . . . . . . .

6.17 Picture of the Sonic Mill. To the left is the bit, at the top right is a motor used to position the bit. Bottom left is a bucket with the slurry. Bottom right are the two controller boxes for the system. .

7.1 Nanowire drawn in Comsol Multiphysics with a length of $600 \mathrm{~nm}$ and a radius of $30 \mathrm{~nm} \ldots \ldots \ldots \ldots$. . . . . . . . . . 74

7.2 Meshed nanowire consisting of 3023 elements . . . . . . . 76

7.3 Displacement of nanowire when a pressure of $40 \mathrm{MPa}$ is applied to the top end of the nanowire . . . . . . . . . . . 76

7.4 Electric potential generated in nanowire when a pressure of 40 MPa is applied to the top end of the nanowire . . . . . . . 77

7.5 Displacement of nanowire with 11645 mesh elements . . . . . . 77

7.6 Electric potential generated in nanowire with 11645 mesh elements 78

7.7 Displacement of nanowire when a pressure of $80 \mathrm{MPa}$ is applied to the top end of the nanowire . . . . . . . . . . . . . 80

7.8 Electric potential generated in nanowire when a pressure of 80 MPa is applied to the top end of the nanowire. . . . . . . . . 81

7.9 Two nanowires spaced $1 \mathrm{~nm}$ apart . . . . . . . . . . . 82

7.10 Two nanowires spaced $1 \mathrm{~nm}$ apart with pressure applied to the left nanowire, which causes the bending of both wires . . . . . . . 
8.1 Measuring the height of nanowires at the top of a scan. . . . . . 88

8.2 Measuring the height of nanowires in the middle of a scan. . . . . 89

8.3 Measuring the height of nanowires at the bottom of a scan. . . . . 90

8.4 Stat-Ease screenshot of graph of Temperature and Ar-flow. . . . . 94

9.1 Schematic representation of manufacturing steps . . . . . . . . . 98

9.2 Si substrate with layer of gold deposited on top. . . . . . . . . . . 100

9.3 Si substrate with annealed gold layer. . . . . . . . . . . . . . . . . 101

9.4 $\mathrm{ZnO}$ nanowire sample from Professor Wang at Georgia Institute of Technology. . . . . . . . . . . . . . . . . . . . 102

10.1 Typical nanowire growth as measured by AFM . . . . . . . . . 108

10.2 Typical nanowire growth picture taken by optical microscope . . 108

10.3 Picture of the finished sensor . . . . . . . . . . . . . . . . . 109

10.4 Circuit diagram of amplifier used to amplify voltage . . . . . . . 110

10.5 Output waveform of pressure sensor when pressure is applied . . 111

10.6 Voltage vs pressure graph . . . . . . . . . . . . . . . 112

10.7 Voltage vs pressure graph for three extra sensors . . . . . . . . . 113

10.8 Output voltage of sensor when pressure is applied at different locations on the sensor. . . . . . . . . . . . . . . . . 115

A.1 AFM picture of a $20 \mathrm{~nm}$ layer of gold deposited on a Si substrate. 135

A.2 AFM picture of $\mathrm{ZnO}$ NWs grown via VLS, with a length of about $200 \mathrm{~nm}$. The parameters for growth were as follows: Time: 30 min, Temperature: $800^{\circ} \mathrm{C}$, Gold Layer: $25 \mathrm{~nm}$ and Ar flow rate: 80 sccm. . . . . . . . . . . . . . . . . . . 136

A.3 AFM picture of $\mathrm{ZnO}$ NWs grown via VLS, with a length of about $120 \mathrm{~nm}$. The parameters for growth were as follows: Time: 40 min, Temperature: $700^{\circ} \mathrm{C}$, Gold Layer: $20 \mathrm{~nm}$ and Ar flow rate: $120 \mathrm{sccm} \ldots \ldots \ldots \ldots \ldots \ldots$

A.4 AFM picture of $\mathrm{ZnO}$ NWs grown via VLS, with a length of about $150 \mathrm{~nm}$. The parameters for growth were as follows: Time: 10 min, Temperature: $1000^{\circ} \mathrm{C}$, Gold Layer: $20 \mathrm{~nm}$ and Ar flow rate: $60 \mathrm{sccm} \ldots \ldots \ldots \ldots \ldots \ldots \ldots$ 
A.5 AFM zoomed-in picture of $\mathrm{ZnO} N W$ s grown via VLS, with a length of about $150 \mathrm{~nm}$. The parameters for growth were as follows: Time: $10 \mathrm{~min}$, Temperature: $1000^{\circ} \mathrm{C}$, Gold Layer: $20 \mathrm{~nm}$ and $\mathrm{Ar}$ flow rate: $60 \mathrm{sccm} . \ldots \ldots \ldots$. . . . . . . . . . . .

A.6 AFM picture of $\mathrm{ZnO}$ NWs grown via the CVD method. The seed layer was deposited from a solution with a concentration of 0.05 $\mathrm{M}$. Growth was achieved from a solution with a concentration of $0.01 \mathrm{M}$, at $95^{\circ} \mathrm{C}$ for $5 \mathrm{~h} . \ldots \ldots \ldots \ldots \ldots$

A.7 AFM picture of $\mathrm{ZnO}$ NWs grown via the CVD method. The seed layer was deposited from a solution with a concentration of 0.05 $\mathrm{M}$. Growth was performed from a solution with a concentration of $0.02 \mathrm{M}$, at $95^{\circ} \mathrm{C}$ for $3 \mathrm{~h}$. . . . . . . . . . . . . . . . 138

A.8 AFM picture of $\mathrm{ZnO}$ NWs grown via electrodeposition, with a length of $380 \mathrm{~nm}$. The parameters for growth were as follows: Temperature: $70^{\circ} \mathrm{C}$, Time: $3 \mathrm{~h}$, Concentration: $0.1 \mathrm{M}$, Current: 0.8

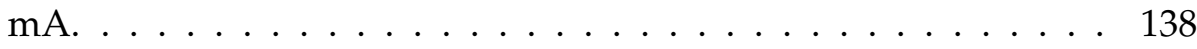

A.9 Light microscope picture of Figure A.7. The dark area is a bundle of grown $\mathrm{ZnO}$ NWs. . . . . . . . . . . . . . . . . . . . . . 139

A.10 Light microscope picture of ZnO NWs grown via CVD method. The parameters for growth were as follows: $0.01 \mathrm{M}$ concentration for growth at $95^{\circ} \mathrm{C}$ for $21 \mathrm{~h}$. The NWs did not align vertically, but rather grew in any direction. The small black rectangles in the picture are ZnO NWs. . . . . . . . . . . . . . . . . . . . . 140

A.11 SEM picture of the growth described in Figure A.10. . . . . . . . . 141

A.12 SEM picture of growth described in Figure A.8. . . . . . . . . . 142

B.1 Timing Schedule for 2009 and $2010 \ldots \ldots \ldots \ldots$ 


\section{List of Tables}

2.1 Top ten nano-related products in 2003 and $2005 \ldots \ldots$. . . . . 9

5.1 Temperature profile of the furnace $(0 \mathrm{~mm}$ is in the centre of the

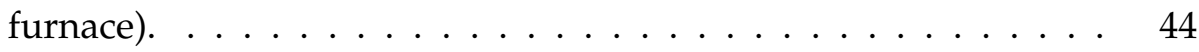

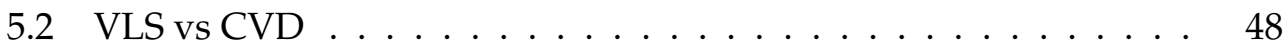

7.1 Relationship between number of mesh elements, electric potential and simulation time . . . . . . . . . . . . . 78

7.2 Relationship between applied pressure, maximum displacement and maximum electric potential . . . . . . . . . . . . . . 79

8.1 Different factors that influence the growth of nanowires . . . . . 86

8.2 Most important factors with their respective levels . . . . . . . . . . 86

8.3 Different factors that were kept constant during each run . . . . . 87

8.4 Experimental parameters . . . . . . . . . . . . . . . . . . . 87

8.5 Statistical results . . . . . . . . . . . . . . . . . . 93

10.1 Different parameters and their values as used for $\mathrm{ZnO}$ nanowire growth . . . . . . . . . . . . . . . . 108

10.2 Pressure exerted on sensor via different weights. . . . . . . . . . . 111

10.3 Superposition of output voltage. . . . . . . . . . . . . . . . 114

10.4 Output voltage of sensor with pressure applied on different areas of sensor. . . . . . . . . . . . . . . . . . . . . . . 114 


\section{Chapter 1}

\section{Introduction}

Nanotechnology is widely researched throughout the world. Countries are spending millions of dollars on nanotechnology, with the United States of America leading the way with USD 8.6 billion in 2008 [16]. Nanotechnology promises to bring cleaner drinking water [17], better medicine and healthcare [18], better cosmetic products [19] and massive data storage [20], to name just a few future nano-related products. Nanotechnology will have an impact on almost every industry we know today. It will drastically change our everyday lives and improve them beyond our imagination.

Nanotechnology research has increased over the last couple of years. Research in nanotechnology was also started in South Africa when the Department of Science and Technology launched the National Nanotechnology Strategy [21]. Stellenbosch University is conducting research in nanotechnology in several academic departments. At the Department of Electrical and Electronic Engineering a fully equipped nano-laboratory is in use [22].

Continuously monitoring pressure is becoming more crucial in certain applications. Nanotechnology can make continuous pressure monitoring possible without an external power source. A pressure sensor which works by exploiting the piezoelectric effect can monitor pressure and display the data without the need of an external power source. Piezoelectricity is electricity generated when pressure is applied to certain materials. The material must have a specific crystal structure. When pressure is applied, the crystal structure loses its symmetry and electricity is generated. The more the applied pressure, the more electricity is generated. Using nanowires instead of the bulk material offers more surface area to work with. 
There are only a couple of materials that exhibit the piezoelectric effect. The number of suitable materials drops even more when nanowires come into play. In this study the most suitable material found was zinc oxide. $\mathrm{ZnO}$ exhibits the piezoelectric effect and there are multiple methods to grow $\mathrm{ZnO}$ nanowires. This made $\mathrm{ZnO}$ the best material to use for the pressure sensor. This thesis will give a complete description of all the work done on nanotechnology pressure sensors, ranging from a literature study to all the practical work and presenting measurements and conclusions.

The Chapter 2 looks at nanotechnology as a whole. A definition is found for nanotechnology and a brief look at the history and the future of nanotechnology is presented. The Chapter 3 considers pressure sensing. The history of pressure sensors as well as the different types of pressure sensing devices are discussed. Chapter 4: Piezotronics, discusses piezoelectricity in more depth. Piezoelectricity on the nanoscale is also discussed and some novel devices are shown. The basic concepts of the pressure sensor are also given, with a complete mathematical description. Chapter 5 gives a brief overview of nanostructures; from their synthesis to the uses of different types of nanostructures. The main focus is on nanowires, as used in this project. All the different methods for growing $\mathrm{ZnO}$ nanowires are discussed. These first five chapters together cover the literature studied to understand nanotechnology, pressure sensing and a combination of the two. Chapter 6 looks at the different equipment available in the nano-laboratory. A brief description of each instrument is given. Chapter 7 shows simulations that were performed to verify the theory described in Chapter 4 . Specialised simulation software was used to simulate a $\mathrm{ZnO}$ nanowire with all its piezoelectric properties. The nanowire bends due to an applied pressure and the corresponding output voltage was then retrieved. Different scenarios were taken into account; from thicker and longer nanowires to higher applied pressures. This gave a good basis for understanding how the sensor should behave in practice. Chapter 8 describes the start of the experimental work. Chapter 5 looked briefly at how the nanowires are grown and now there after in Chapter 8 the actual growing of the nanowiress is reported. The vapour liquid solid (VLS) method was chosen to grow the nanowires and the optimisation of this method is discussed. A statistical method was followed in order to obtain the optimal parameters to grow the best nanowires. Chapter 9 looks at the manufacturing process of the pressure sensor, from the growth of the 
nanowires to wirebonding. Each step in the manufacturing process is discussed in detail. Chapter 10 shows the measurements and results from use of the pressure sensor. Different pressures were applied to a sensor and the corresponding output voltages were found and plotted. The results are then discussed in full detail. The last chapter draws the entire project to a conclusion. All the difficulties and problem areas are discussed and potential solutions are given. 


\section{Chapter 2}

\section{Nanotechnology}

\subsection{What is nanotechnology?}

Finding a formal definition for nanotechnology is harder than meets the eye; everyone has their own definition. According to Centre for Responsible Nanotechnology (CRN) the definition is as follows: "Nanotechnology is the engineering of functional systems at the molecular scale. This covers both current work and concepts that are more advanced. In its original sense, 'nanotechnology' refers to the projected ability to construct items from the bottom up, using techniques and tools being developed today to make complete, high performance products" [23]. Nanowerk, an online magazine, has its own definition: "The design, characterization, production, and application of structures, devices, and systems by controlled manipulation of size and shape at the nanometer scale (atomic, molecular, and macromolecular scale) that produces structures, devices, and systems with at least one novel/superior characteristic or property" [24]. The National Nanotechnology Institute (NNI) of America also has its own definition: "Nanotechnology is the understanding and control of matter at dimensions between approximately 1 and 100 nanometers, where unique phenomena enable novel applications. Encompassing nanoscale science, engineering, technology, nanotechnology involves imaging, measuring, modeling, and manipulating matter at this length scale" [25]. The one area where all definitions do agree is that something can be considered nanotechnology if the object under scrutiny has at least one dimension smaller than $100 \mathrm{~nm}$.

At present, as discussed above, there is not a single, universally used for- 
mal definition of nanotechnology. Nano in ancient Greek meant dwarf, and today it is defined as $10^{-9}$. This gives a clear indication that nanotechnology has to do with technology on a very small scale. To put the size in perspective: a human hair is about 17 - $181 \mu \mathrm{m}$ or $17000-181000 \mathrm{~nm}$ in width [26], about 1000 times thicker than nanosized products. The human eye has a CPD (cycles per degree) of 50, which means it can see an object of $0.35 \mathrm{~mm}$ from $1 \mathrm{~m}$ [27]. This again highlights the fact that nanotechnology truly has to do with a very small scale, far below the threshold of human sight.

Nanotechnology is often referred to as a general-purpose technology, because it will have an impact on almost all industries and all areas of society. It offers better built, longer lasting, cleaner, safer and smarter products for the home, for communications, for medicine, for transport, for agriculture and for industry in general [23]. Nanotechnology will not benefit just one industry but will spread much wider and benefit a whole group of industries concerned with anything, from everyday products to specialist products, much like the personal computer that is also present in almost every industry today.

The word nanotechnology was popularized by Eric Drexler in the 1980s [23]. He gave a talk about building machines on the scale of molecules, a few nanometers wide. Drexler made the word popular, but it was in a paper about an ion-sputter machine by Taniguchi that the word nanotechnology was first used, back in 1974 [23].

Nanotechnology may be small in size, but it generates lots of attention. In the USA alone, more than USD 60 billion nano related products were sold in 2004 and this number is set to rise to USD 2.6 trillion by 2014 [28]. It is a highly profitable technology because it is very diverse and has applications in almost every industry, as mentioned above.

\subsection{History of nanotechnology}

Nanotechnology is a very new field, although traces of nanotechnology can be found hundreds of years ago, even as far back as Roman times and earlier. Glassmakers used the color changing properties of nanotechnology to create masterpieces, but they did not know how it worked and they had never heard of nanotechnology. When a match is lit, fullerenes are produced - a carbon allotrope. Carbon black was produced in the 1920s without their knowledge. This substance is what makes car tyres black and it decreases tyre wear, 
but this was not fully understood back then [28].

Nanotechnology really started in 1959 when Richard Feynman gave is well known talk: "There is Plenty of Room at the Bottom" [24]. Feynman wanted to write the entire Encyclopedia Britannica on a pin head. He wanted to do this by manipulating individual atoms by making small factories that will be able to write down everything on a needle head. Although Feynman never mentioned nanotechnology during the speech, most people regard this as the start of the nanotechnology era [24].

No experiments in nanotechnology had been done before Feynman's speech, nor for a couple of years after it, because the necessary tools did not exist. Scientists were not able to see individual atoms until years later. In 1974 Taniguchi used the term "nano-technology" for the first time in his paper about the ion-sputtering machine [23]. Then in 1977 Drexler started work at Massachusetts Institute of Technology (MIT) on molecular nanotechnology, but it was still only concepts and did not yield any devices or equipment. The first big step came in 1981 when the first scanning tunneling microscope (STM) was invented at IBM by Gerd Binnig and Heinrich Roher, who shared the Nobel Prize in 1986 [29]. For the first time scientists could see down to the nanoscale. 1981 also saw the first paper on engineering on the atomic level. In 1985 buckyballs were discovered - fullerenes that are spherical. 1986 saw the first book on nanotechnology published, "Engines of creation: The coming era of nanotechnology". The atomic force microscope (AFM) was also invented in 1986. In 1987 the first university symposium on nanotechnology was held at MIT and in 1988 Drexler gave a ten-week course, "Nanotechnology and Exploratory Engineering" at Stanford, the first classes regarding nanotechnology [30]. In 1989 IBM spelled I-B-M with individual atoms as seen in Figure 2.1 [1]. In 1990 the first nanotechnology journal named "Nanotechnology" appeared. In 1991 carbon nanotubes were discovered by Sumio Iijima, 1992 the first textbook was published, "Nanosystems: Molecular Machinery, Manufacturing, and Computation" [30]. From this point nanotechnology really took off, with new inventions and large strides forward being made year on year. In the year 2000 President Clinton of the USA announced the U.S. National Nanotechnology Initiative.

Figure 2.2 [2] shows a timeline describing the growth of nanotechnology. Major events in the nanotechnology field are shown. The figure shows that nanotechnology is truly a very new field, with all major events happening 


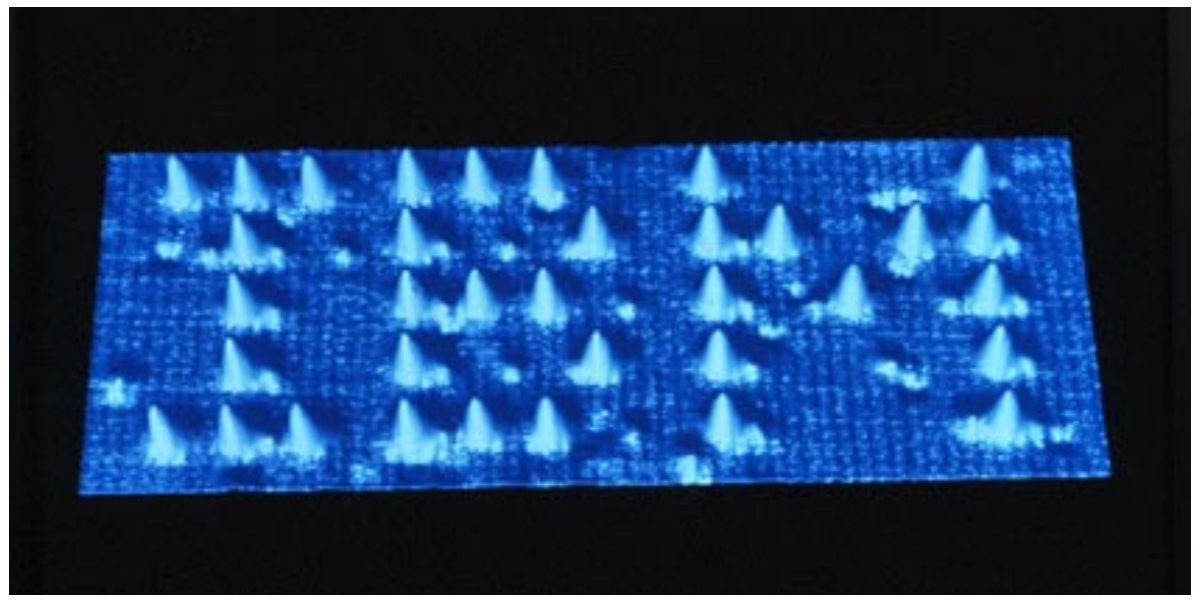

Figure 2.1: IBM written with individual atoms [1]

within the last 20 years. A graphic and more complete timeline can be viewed at [31].

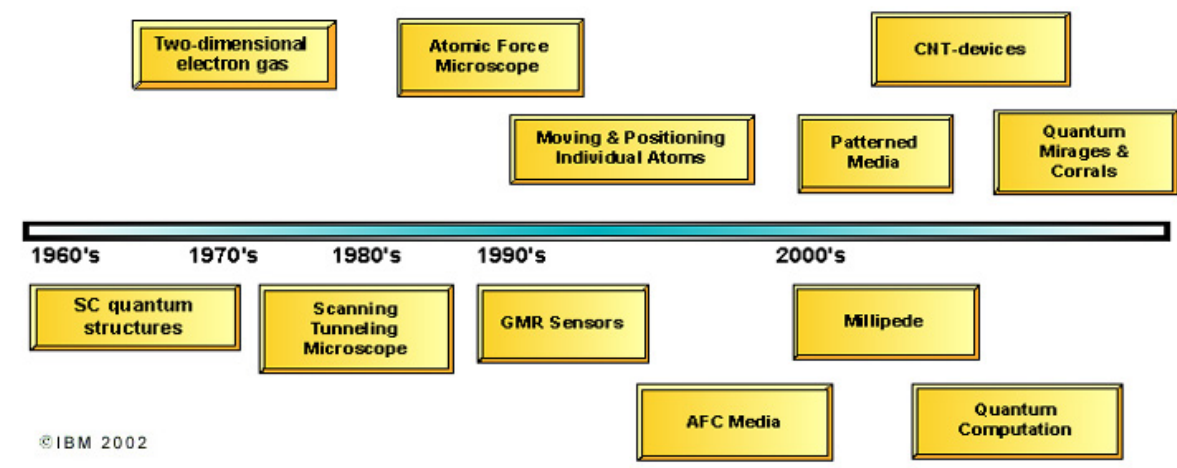

Figure 2.2: Timeline for nanotechnology [2]

\subsection{Future of nanotechnology}

As with any new technology, the success lies with the public acceptance and commercial usefulness. When the public decide they want a certain product, they will buy it and the product will become a success. Conversely, when the public has a negative feeling toward a product they will not buy the product 
in question and thus the product will be a failure. In a recent survey it was found that most Americans are against nanotechnology because they believe it is immoral and against their religion [32]. The probable reason for the lack in support for nanotechnology is because most people do not really know what nanotechnology is and what it can do for them. From stain resistant clothes to windows that clean themselves, to more efficient solar panels, to cancer fighting drugs, the possibilities are endless. New technologies are almost always viewed with a bit of scepticism, but when the new technology proves itself and makes a difference, people will start to accept it. People are always afraid to change their lives and will not easily adopt new technology; this gets worse if the new technology is hard to understand.

Nanotechnology is already part of a number of products we use on an everyday basis. Sunscreen makes use of small nano-sized particles that block the sun's UV-rays. Computers use lines with a width of only $45 \mathrm{~nm}$ to transfer data from one point to another. Stain resistant clothes are made of a fabric that has nano-sized fibers to prevent stains from accumulating on the surface of the fabric. Most people do not even know that they are already reaping the benefits from nanotechnology and when asked, they will be against nanotechnology.

New rules and regulations must be set up in order to prevent people from using nanotechnology in any way that could harm others. Because nanotechnology is so diverse it would be easy to use the technology to have a negative impact on a large scale. In America there are already a large number of laws governing nanotechnology [33].

One of the main areas of concern is in the medical or biomedical field. For all the good nanotechnology can do, it can do just as much harm. Thorough testing will be necessary before any nanotechnology products are implanted into humans. Human safety should never be viewed as less important than profit, but should always be the most important factor when employing technologies that can benefit humans.

Nanotechnology can change the world in the 21st century, as the computer changed the world in the 20th century. It has a lot of potential and can make safer, more reliable, faster and smaller products. There are many products that already incorporate nanotechnology. According to Forbes magazine the top ten nanotechnology products in 2003 and 2005 were [34], [35]:

Looking further than that, everyday products that utilise nanotechnology 
Table 2.1: Top ten nano-related products in 2003 and 2005

\begin{tabular}{|c|c|}
\hline $\mathbf{2 0 0 3}$ & 2005 \\
\hline High-Performance Ski Wax & Tiny Chips - Apple Computer's iPod Nano \\
\hline Breathable Waterproof Ski Jacket & Shemen Industries Canola Active (Cooking Oil) \\
\hline Wrinkle-Resistant, Stain-Repellent Threads & O'Lala Foods Choco'la Chewing Gum \\
\hline Deep-Penetrating Skin Cream & Zelens Fullerene C-60 Face Cream \\
\hline World's First OLED Digital Camera & Easton Sports StealthCNT Bat \\
\hline Nanotech DVD and Book Collection & Nanotex - Mattress cover \\
\hline Performance Sunglasses & ARC Outdoors, ArcticShield Socks \\
\hline Nanocrystalline Sunscreen & Behr NanoGuard Paint \\
\hline High-Tech Tennis Rackets & Pilkington Activ Glass \\
\hline High-Tech Tennis Balls & NanoBreeze Air Purifier \\
\hline
\end{tabular}

include: tennis balls and tennis rackets, golf balls, ski wax [36], anti-aging cream, certain food products, packaging [37] and the list goes on. It can be seen from these lists that nanotechnology has already penetrated our everyday lives and that the uses are positive. 


\section{Chapter 3}

\section{Pressure Sensors}

\subsection{What is Pressure?}

Pressure is an important parameter in many industrial products as well as everday products. Car tyre pressure plays a big role in determining the lifespan of your tyres. The more heavily you load your car, the higher the tyre pressure should be [38]. Industrial gas tanks operate at a certain pressure and can explode if the pressure suddenly drops or rises. Monitoring of pressure is thus crucial in some situations and can help in many different ways. Pressure can be measured with a number of different methods and there are also different units in which pressure is measured.

The SI unit for pressure is Pascal (Pa) and 1 Pa equals a force of one Newton per square meter $\left(1 \mathrm{~Pa}=1 \mathrm{~N} / \mathrm{m}^{2}\right)$ [39]. Translated, this means that pressure is the force that is applied to a certain area, the higher the force, the higher the pressure. At sea level the pressure of the earth's surface is atmosphere, $101325 \mathrm{~Pa}$ or $101.325 \mathrm{kPa}$, and this equals one atmosphere of pressure (atm). In many applications it is necessary to know the working pressure, as mentioned above. If the difference between the pressure inside the tank and outside it is too big, the tank could either be crushed are explode. This simple example shows why it is so important for pressure to be measured accurately. 


\subsection{History of Pressure Measurements}

In 1594 Galileo Galilei built a machine to pump water from a river. The heart of this machine was a syringe. He found that the limit to which the syringe would fill was about $10 \mathrm{~m}$. Although the syringe could take more water, it would not fill up beyond the $10 \mathrm{~m}$ mark and he could not explain why. In 1644 Evangelista Torricelli filled a tube of about one meter, closed at one end, with mercury, then inverted the tube and placed the open end in another vessel, also filled with mercury. The mercury in the long tube then fell to about $760 \mathrm{~mm}$, leaving an empty space above the level of the mercury column. The mercury dropped from the level at the top of the tube mark to about 760 $\mathrm{mm}$, leaving a $240 \mathrm{~mm}$ of space above the mercury. Torricelli attributed the phenomenon to an unknown force on the surface of the mercury in the open vessel, and thus on everything of earth. In 1648 Blaise Pascal heard about Galilei's and Torricelli's experiments and he went searching for answers. He found that the force that held the column of mercury at a certain level inside the tube was the weight of the surrounding air and from this he calculated the weight of air. Pascal formulated that this force, which he called atmospheric pressure, acts uniformly in all directions [40].

\subsection{Different Pressure Measurement Equipment}

There is more than one method of measuring pressure. The type of pressure you want to measure will determine what sort of equipment you will use. The following are a few methods that can be used to measure pressure:

\subsubsection{Sight Glass}

The simplest and oldest method to measure pressure is through a sight glass. It is a transparent tube through which one can see the level of liquid inside the tube [3]. This is basically what Torricelli used for his experiment. This method has a number of drawbacks: The transparent tube can fail, the liquid inside can be dirty, making it hard to see the precise level, and leaks can occur, to name just a few. Placing a buoyant object, of a specific mass, in a tube filled with liquid and air can also be used to determine pressure. The level at which the buoyant object floats indicates the pressure. This is an ancient method and is rarely used today. 


\subsubsection{Magnetic Pressure Sensing}

A magnetic level gauge works on the buoyant object principle. It works on the same principal as the sight glass method, but instead of having to see where the liquid level is, some buoyant object determines the level. Instead of just reading the level of the buoyant object on a scale, the position is determined magnetically. The float, or buoyant object, is a strong magnet and a magnetized shuttle is dragged along as the float moves up and down, as can be seen in Figure 3.1 [3].

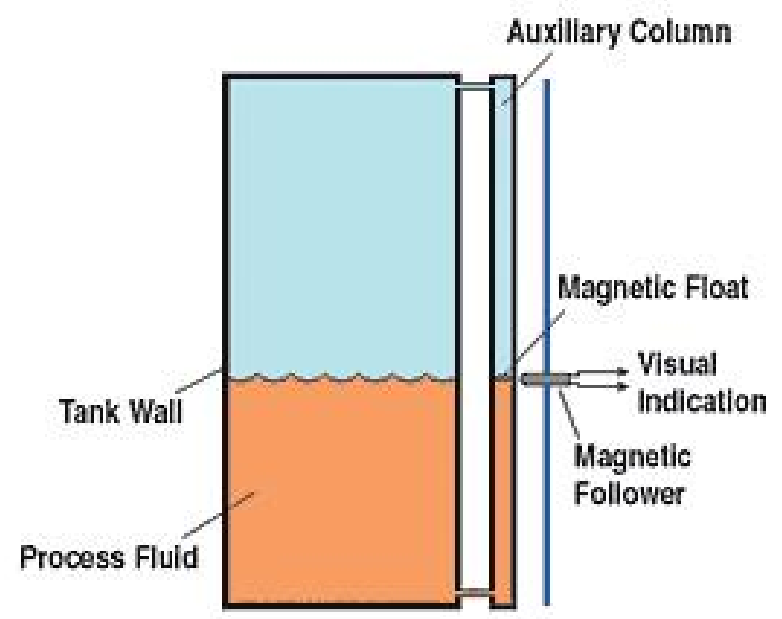

Figure 3.1: Schematic of Magnetic Pressure Sensor [3]

\subsubsection{Capacitive Pressure Sensing}

Fluids have dielectric constants, a measure of the extent to which the fluid concentrates electrostatic lines of flux. The dielectric constant is the determining factor in designing capacitors [41]. Pressure measurements using capacitance make use of the change in capacitance as the liquid level varies. The capacitance is created by a rod that is attached to a reference probe and a transmitter. As the liquid level rises, the capacitance will also rise. Figure 3.2 shows how it works [3]. The same principle can be used for resistive measurements. A fluid has a specific resistive value if a current flows through it and the more fluid there is, the higher the resistance. By measuring the resistance of the fluid the pressure can be calculated [3]. 

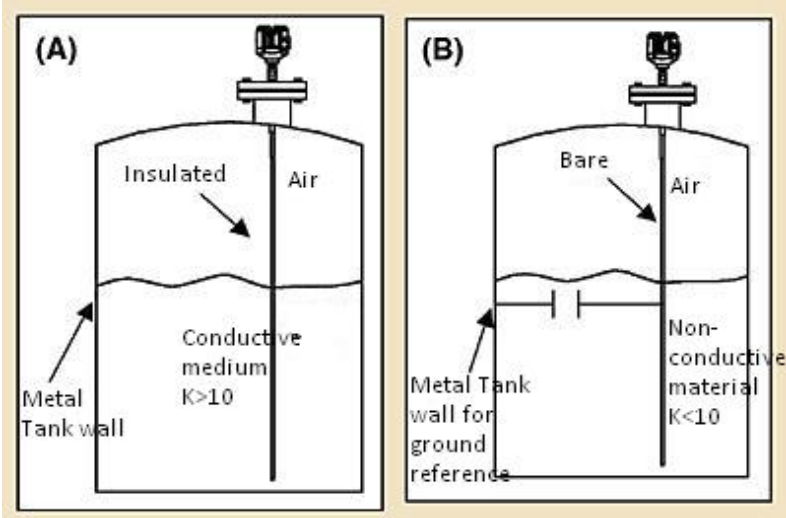

Figure 3.2: Schematic of Capacitive Pressure Sensor [3]

\subsubsection{Piezoresistive Pressure Sensing}

Piezoresistive materials are materials that change their resistance to the flow of current when the materials are compressed or strained. Most piezoresistive materials are made from semiconducting silicon. When a force is applied to the silicon, it becomes more resistant to a current traveling through it. A piezoresistive pressure sensor contains several thin wafers of silicon embedded between protective surfaces. The device is connected to a system that can measure minute differences in resistance. The system will send a small current through the sensor and measure the resistance. This process will be repeated and, on the second run, if a force was applied, the resistance will have changed and the difference in resistance can be used to calculate the pressure [42].

\subsubsection{Nano-sized Pressure Sensor}

For this project, pressure at a very small scale must be measured. It is thus impractical to use the sight glass method or any other method where one needs to physically see the pressure level. Something that changes a value as the pressure changes is the only method that can work on such a small scale. The three choices investigated for this project were the capacitive, resistive and piezoresistive methods. The capacitive or resistive methods can work, but again, on the nanoscale the changes will be so minuscule that very sensitive and large measuring equipment would be necessary, defeating the purpose of building a small pressure sensor. The other problem is that an 
external power source must be available to send a current through a material in order to measure the resistance. Although this is also true for the piezoresistive method on a large scale, this is not so on a nanoscale.

The piezoelectric effect, as described in full in Chapter 4, is what occurs when the bending of a specific material generates a voltage. The more the material bends, the more the voltage would be. This works on the same principal as the piezoresistive method, ie. if the resistance increases, the pressure increases. Only certain materials exhibit this effect and the reasons for this will be discussed in Chapter 4. Because of the piezoelectric effect, an external voltage source for a pressure sensor, will not be necessary, as it generates its own voltage when pressure is present. Neither is sensitive measuring equipment required, because the voltage that is generated can be picked up by most multimeters or similar equipment. These were the main reasons for choosing this method for building a pressure sensor on the nanoscale. Without an external voltage source the size could be kept at a minimum, and the sensor can truly be a nano-sized sensor.

Developing a sensor based on nanowires to measure pressure has a number of advantages. The pressure is converted to a voltage signal, due to the piezoelectric effect; this voltage can be recorded and then used to power a transmitter to send the pressure value wirelessly to a receiver. This can be used in remote or dangerous areas to continuously monitor pressure without the need to replace batteries. 


\section{Chapter 4}

\section{Piezotronics}

\subsection{What is Piezotronics?}

Piezoelectricity is electricity that is generated by the piezoelectric effect. The piezoelectric effect involves the generation of electricity by the bending of certain crystals. The reverse piezoelectric effect is also observed in some crystals when an applied voltage causes bending in the crystal. Piezoelectricity is related to pyroelectricity, where electricity is generated by heating or cooling a crystal [43]. Piezo in Greek means to squeeze or to press.

The piezoelectric property is dictated by the manner in which the atoms of the crystal align themselves. The crystal consists of multiple interlocking domains which have positive and negative charges. These domains are symmetrical within the crystal, with the result that the crystal has a net charge of zero [44]. When a stress is applied to the crystal this symmetry is broken, and in order to restore the symmetry these domains realign themselves, and through the realignment, generate a voltage. Many crystalline materials exhibit the piezoelectric effect, including quartz, wurtzite-structured crystals, Rochelle salt, lead zirconate titanate ceramics, barium titanate and polyvinylidene fluoride [4]. When such a crystal is deformed, the positive charge centres and the negative charge centres are displaced with respect to one another as illustrated in Figure 4.1 [4]. Overall the crystal remains electrically neutral, but the difference in the charge centres results in an electric polarization inside the crystal.

The converse piezoelectric effect works on the opposite principal. The crystal atoms are aligned and have a zero charge. When a voltage is applied 
over the crystal, the symmetry is broken because of the electricity that flows through the crystal. In order to restore the zero charge, the crystal deforms.

Piezoelectricity is used in sensors, actuators, clocks, lighters and transducers [43]. However, the most common use of piezoelectricity is in a loudspeaker that converts electrical signals to sound by deforming a crystal. The crystal never deforms more than a few nanometers, but the force exerted on the crystal to induce the deformation is in the order of a few meganewtons [43]. For example, a one cubic centimeter cube of quartz with a two kilonewton applied force produces $12 \mathrm{kV}$, but at a low current [45]. Because the force needed is so great to cause just a few nanometers of deformation it was reasoned that working on the nanoscale could improve the generation. A small crystal, with dimensions of only a few nanometers, being displaced by a few nanometers, will not require a great force to induce the displacement.

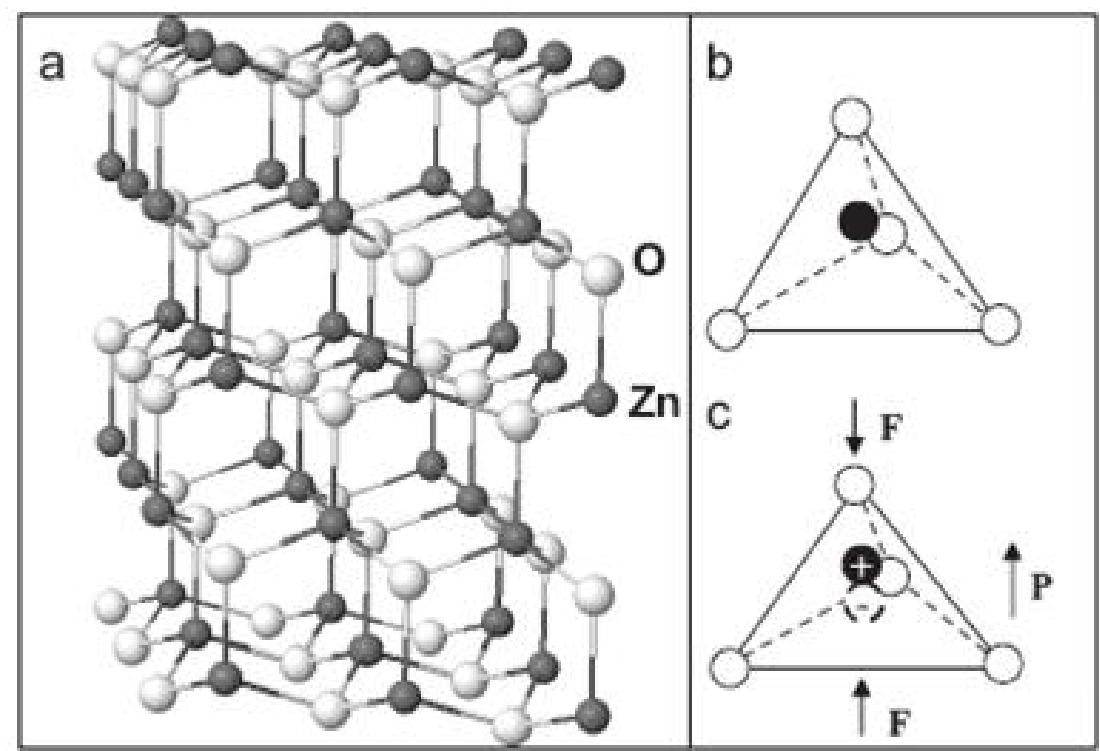

Figure 4.1: a) Crystal structure of $\mathrm{ZnO}$. b) Tetrahedral coordination between $\mathrm{Zn}$ and O. c) External force displaces the positive charge in relation to the negative charge, causing a potential difference [4]. 


\subsection{History of Piezotronics}

The piezoelectric effect was discovered in 1880 by Jacques Curie when he published a paper about an experiment demonstrating a connection between macroscopic piezoelectricity and crystallographic structures. The experiment consisted of measuring the surface charges appearing on specially prepared crystals which were subjected to mechanical stress [46]. Curie thought the stress applied to the crystal had an effect on the temperature of the crystal and thus that the temperature change was the cause of the electricity that was produced, in other words, that it was pyroelectricity [45]. Lippmann, in 1881 , showed that the stress applied to the crystal was the contributing factor in the generation of electricity. He also showed, and explained, the converse piezoelectric effect.

From 1881 to 1910 a lot of work was done on piezoelectricity and 20 naturally occurring crystals were shown to be piezoelectric [46]. In 1910 Voigt's $^{\prime}$ "Lerbuch der Kristallphysik" became the standard reference necessary to understand piezoelectricity [44] [46]. During the First World War, in 1917, the French used the piezoelectric effect to build an ultrasonic submarine detector [47]. Their transducer was a thin quartz crystal glued between two steel plates. Working on past the end of the war, they did achieve their goal of emitting a high frequency "chirp" underwater and measuring depth by timing the return echo [46]. After the successful testing by the French, piezoelectric devices got a lot of attention. New products were developed during the 1930s, ranging from oscillators, to ultrasonic wave generators, to pressure measuring equipment [46]. The Second World War saw major advances in piezoelectric devices. The US, Japan and the Soviet Union had isolated groups working on piezoelectric devices [48]. The advances made during this time fall into three categories:

- Development of the barium titanate family of piezoceramics,

- understanding of crystal structures and the electro-mechanical activity, and

- doping of materials to achieve desired properties, such as piezoelectricity [46].

These advances once again saw a number of new products, from powerful sonar, to piezo ignition systems, microphones and relays [46]. All of the 
above mentioned products and developments were made by Americans, but the work had a cloud of secrecy surrounding it. Everyone kept their findings and working methods of their products to themselves, thus haltering productivity. The Japanese had an open and cooperative approach and this saw them making even better headway on the piezoelectric front. They created new piezoceramics families [48], and they also made a number of new products, from audio buzzers to filters [46]. Because everyone working on piezoelectricity shared their insights, progress was made much faster than in the rest of the world. During the last couple of years most researchers have adopted the Japanese way and are sharing all their information; this is opening the door for more piezoelectric devices. New technologies such as nanotechnology are also benefiting from piezoelectronics. A whole new field, nanopiezotronics, was invented in 2006 by Prof Zhong Lin Wang [49].

\subsection{Nanopiezotronics}

Nanopiezotronics is a very new field in nanotechnology. Nanopiezotronics was introduced by Professor Zhong Lin Wang of the Georgia Institute of Technology in 2006 [49]. Nanopiezotronics utilizes the coupled piezoelectric and semiconducting properties of nanowires and nanobelts [49] [4] [5]. Devices being manufactured using nanopiezotronics have applications ranging from field effect transistors [4], diodes [4] to nanogenerators [49]. Nanopiezotronics will also have a wide range of applications in electromechanical coupled electronics, sensing and in harvesting energy from the environment and self-powered nanosystems [4].

\subsubsection{Field Effect Transistor}

A field effect transistor (FET) made from nanowires or nanotubes is a commonly studied device in nanotechnology [4]. A typical FET is composed of a semiconducting nanowire, connected by two electrodes at its ends, which is then placed on a silicon substrate covered by a thin layer of gate oxide. A third electrode is then built between the nanowire and the gate oxide, as seen in Figure 4.2 [5].

The gate voltage $\left(V_{G}\right)$ can trap and deplete carriers in the nanowire. By controlling the gate voltage, the flow of electrons from source to drain can be controlled [4]. The nanopiezotronic FET works on very much the same 


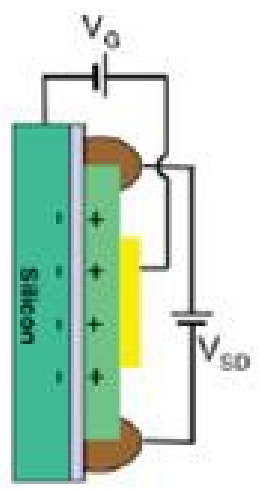

Figure 4.2: Schematic diagram of FET [5]

principle. The nanowire is still attached to two electrodes, but the gate is omitted. The nanowire is then exposed to the environment. If a pressure is applied to the nanowire, the piezoelectricity across the nanowire acts as the gate that controls the current flowing through the nanowire [4] (Figure 4.3). This is the basic principle of the nanopiezotronic FET.

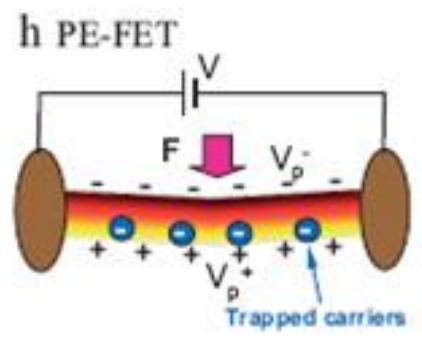

Figure 4.3: Schematic diagram of nano-FET [4]

\subsubsection{Gated Diode}

Another basic electric component that can be realized through nanopiezotronics is a diode, through employing a two probe technique [4]. One probe is held at one end of the nanowire that lies on an insulating surface and the other probe is used to push the nanowire at the other end [5]. The probe has an ohmic contact with the nanowire as seen in the first figure of Figure 4.4, and the I-V curve changes to a linear graph to rectify the behaviour as the 
degree of bending increases, as seen in Figure 4.4. As the tip bends the wire, the contact between the tip and the nanowire changes from an ohmic contact to diode-like behaviour. This behaviour is witnessed, because when the tip bends the nanowire, the nanowire generates a voltage, which changes the I-V curve from linear to diode-like behaviour.

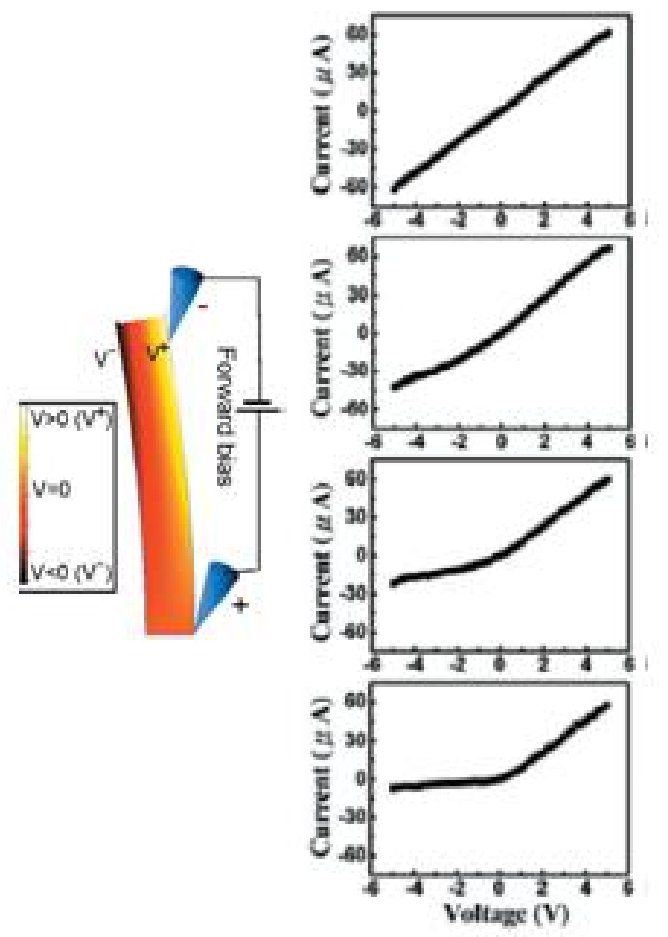

Figure 4.4: Schematic diagram of nano-Gated Diode [5]

\subsection{Nanogenerator}

Combining the above mentioned devices, a FET and a gated diode, it forms the basis of a nanogenerator. As the name implies, a nanogenerator generates its own electricity, but on a nanoscale. With the nano-revolution that is taking place, everyday products will become smaller, but this will not help if they still need to be connected to a power supply or need a large battery attached to it. There is thus a need to make nanodevices self powered. The nanogenerator is aimed at doing just that: make nanodevices that are self 
powered. The nanogenerator was first introduced by Professor Zhong Lin Wang in 2006 [6]. Nanosized devices need to have their own source of power in order to remain nanosized. There is no point in designing a nanosized device just to connect it to a power source magnitudes bigger that the device itself. It is thus necessary to give these nanodevices the ability to harness energy from the environment in order to power them. The nanogenerator has the ability to provide this energy to nanodevices. In Figure 4.5 novel methods to harness the environment for electricity are shown. The rest of this chapter will include a complete discussion of how the nanogenerator works, as well as the mathematical background.

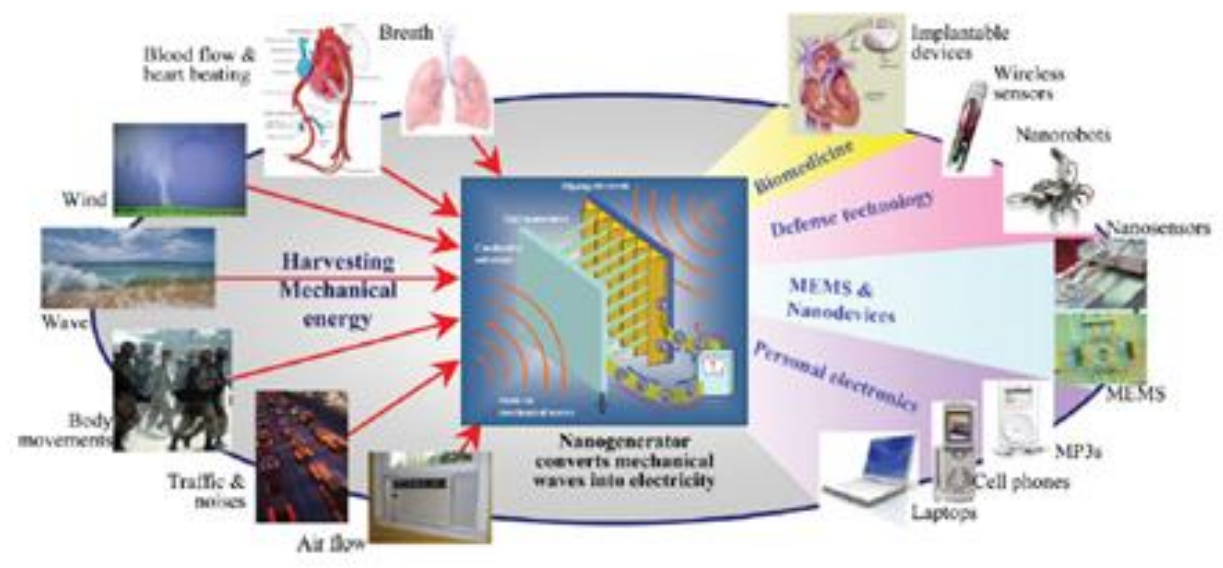

Figure 4.5: Different methods to harness electricity from the environment using a nanogenerator [6].

\subsubsection{Nanogenerator basics}

The nanogenerator makes use of zinc oxide $(\mathrm{ZnO})$ nanowires. The growth of $\mathrm{ZnO}$ nanowires are discussed in full in Chapter 5 . The nanowires are grown on a silicon substrate and are vertically aligned. $\mathrm{ZnO}$ is used because it has a wurtzite structure, in which the $\mathrm{Zn}$ cations and $\mathrm{O}$ anions form a tetrahedral coordination. Wurtzite structures are structures that contain Zn, Fe or S. ZnO has two important characteristics: The presence of polar surfaces and lack of centre symmetry [5]. The interaction of polar surfaces makes the growth of unique $\mathrm{ZnO}$ nanostructures [50] such as nanobelts [51], nanosprings [52], 
nanorings [53] and nanohelics [10] possible. The lack of centre symmetry is what gives $\mathrm{ZnO}$ its piezoelectric ability [5], the displacement of the $\mathrm{Zn}$ cations and $\mathrm{O}$ anions in the crystal. Another important characteristic of $\mathrm{ZnO}$ is that it is a semiconducting material with high bandgap energy of $3.37 \mathrm{eV}$ at room temperature $[54,55]$. The bandgap is an energy area where no electrons can exist. $\mathrm{ZnO}$ has an extremely large exciton binding energy of $60 \mathrm{meV}$ and is very stable at room temperature [54]. Exciton binding energy gives an indication of how stable the electrons are. An electron "binds" with an electron hole and the exciton binding energy shows how good this "'bind"' is. $\mathrm{ZnO}$ has attracted a lot of attention and using the unique electronic, optical, mechanical and piezoelectric properties, novel devices such as field effect transistors [29], ulta-violet (UV) lasers [56], UV photo detectors [57], light emitting diodes (LEDs) [58], solar cells [59], piezoelectric transducers and actuators [60] have been demonstrated. As discussed above, by using the semiconducting and piezoelectric properties of $\mathrm{ZnO}$, piezoelectric FET and piezoelectric diodes were realized.

To explain the piezoelectric phenomenon a vertical, straight $\mathrm{ZnO}$ nanowire, as depicted in Figure 4.6a, is used. The nanowire is deflected by an external force, $F$, which is applied to the nanowire, causing it to bend. This results in stretching of the outer surface and compresson of the inner surface [4] [5]. Due to the piezoelectric effect an electric field is created inside the nanowire, as seen in Figure 4.6c. Approximating the potential generated by the piezoelectric effect, the compressed side is at $V_{-P}$ and the stretched side is at $V_{+P}$. The potential is created by the relative displacement of the $\mathrm{Zn}^{2+}$ cations with respect to the $0^{2-}$ anions because of the piezoelectric effect [4] [5]. The potential difference is maintained as long as the nanowire is deformed, thus while the symmetry is broken. The crystal does realign itself as quickly as possible, which means that the potential is not constant but reaches a maximum value and decreases gradually to reach zero again. This is the basic process for both the FET and diode, as described above.

At this point the potential is still contained within the surface of the nanowire. In order to utilize the potential on the surface of the nanowire, the nanowire must be connected to an external circuit. This is known as the discharge process. For simplicity, the external circuit is a conductive tip of the AFM, and the tip also induces the deformation of the nanowire. When the tip scans over the nanowire it comes into contact with the stretched side of the nanowire, 


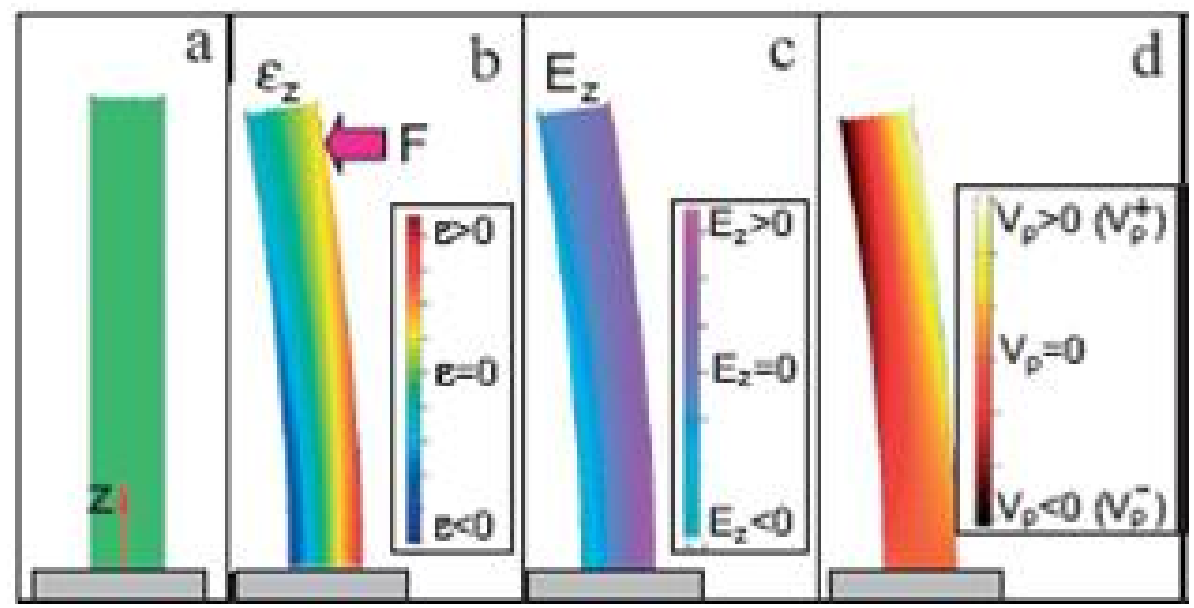

Figure 4.6: a) Visual representation of $\mathrm{ZnO}$ nanowire b) Strain induced in nanowire due to external force c) Electric field due to piezoelectric effect and d) Voltage induced due to the piezoelectric effect [4].

which is at the positive potential $V_{+P}$, as illustrated in Figure 4.7a. The conductive tip has a potential of nearly zero, $V_{m}$, so the tip/ZnO interface is negatively biased, $\triangle V=V_{m}-V_{+P}<0$ [4] [5]. The $\mathrm{ZnO}$ nanowire is an n-type semiconductor which forms a reverse-biased Schottky diode with the conductive tip and a small current flows across the interface [4]. What exactly happens at the Schottky diode interface will be discussed later in the chapter. As the tip moves further over the nanowire, it comes into contact with the compressed side of the nanowire and the tip/ $\mathrm{ZnO}$ interface now becomes positively biased, $\triangle V=V_{m}-V_{+P}>0$ as illustrated in Figure 4.7 [4] [5]. The interface forms a positively biased Schottky diode which produces a sudden increase in the current that flows across the interface [4]. The current is a result of the $\triangle V$ potential flow of electrons from the semiconductor to the conductive tip. The flow of the free electrons from the nanowire to the tip will neutralize the ionic charges distributed inside the nanowire, thus reducing the potentials at either sides of the nanowire.

The charge-releasing process at the Schottky diode is a very important and necessary process in the operation of the nanogenerator. The nanowire forms a Schottky contact with the AFM tip with a barrier height of $F_{S B}$ and forms an Ohmic contact with the ground, as depicted in Figure 4.8. When the tip deforms the nanowire, electrons flow from the ground through an external load (the AFM in this case) to reach the tip. The electrons cannot cross 

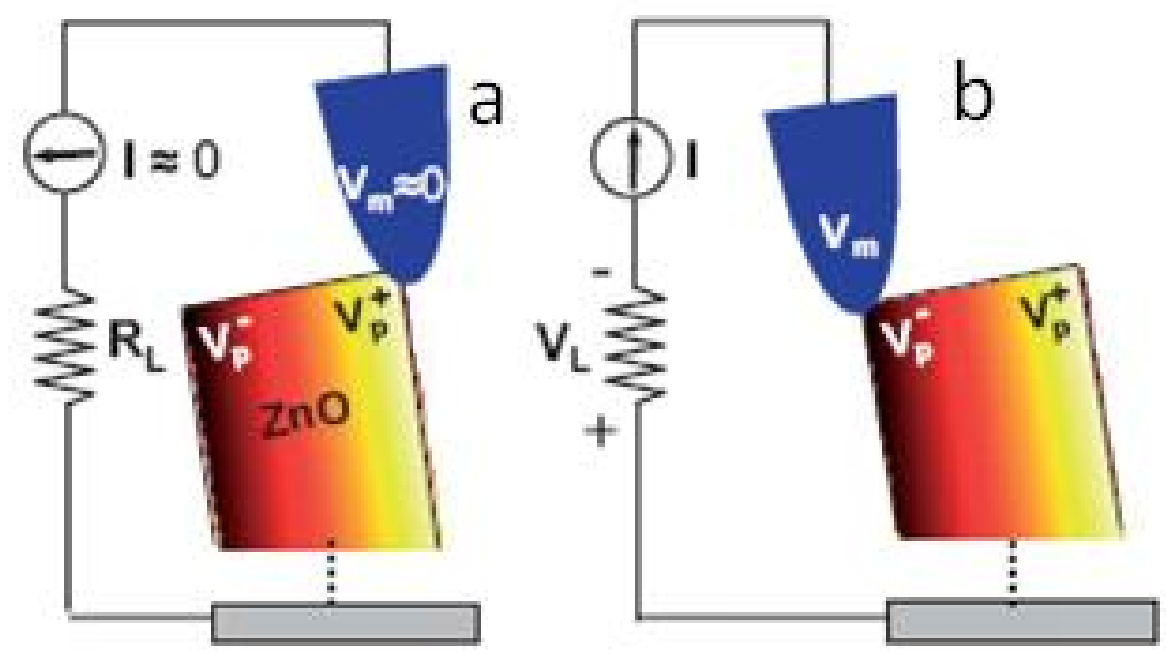

Figure 4.7: a) Tip in contact with stretched side of nanowire b) Tip in contact with compressed side of nanowire [4].

the nanowire/tip interface because of the reverse-biased Schottky barrier. The potential created by the flow of electrons, $V_{O P}$, lowers the conduction band (CB) slightly. When the tip scans further and reaches the middle of the nanowire, the piezoelectric potential is zero. The generated potential, $V_{O P}$, suddenly drops to zero because the piezoelectric potential is zero and thus no electrons flow. The electrons that flowed when the tip was first in contact with the nanowire will now flow back through the tip, through the load and back to ground. When the tip then scans further and touches the compressed side of the nanowire, the potential, $V_{O P}$, becomes negative, which causes the $\mathrm{CB}$ at the tip to lift. If the potential is large enough to overcome the Schottky barrier threshold, the electrons can flow through the nanowire to the tip. This creates a circular flow of electrons through the external circuit [5] [61].

A Schottky barrier is a barrier that forms between a semiconductor and a metal, that has rectifying characteristics, which makes it ideal to use as a diode. In order to form a Schottky barrier between the nanowire and the AFM tip in this case, or any other tip, the electron affinity of $\mathrm{ZnO}$ must be smaller than the work function of the tip. $\mathrm{ZnO}$ has an electron affinity of $4.5 \mathrm{eV}$, so the tip on the other side must have a work function higher than $4.5 \mathrm{eV}$. Electron affinity is the amount of energy required to detach an electron from a singly charged ion and an element's work function is the minimum 


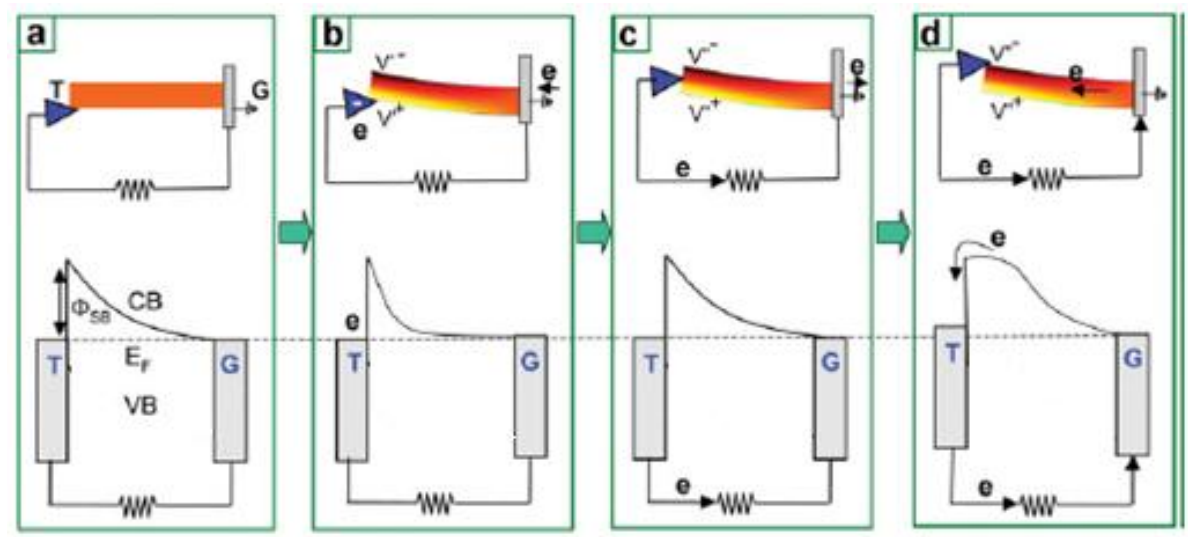

Figure 4.8: a) The tip first comes into contact with the nanowire and forms a Schottky barrier b) The tip causes the nanowire to bend and in turn the nanowire generates a voltage, due to the piezoelectric effect and electrons begin to flow from the ground c) The tip reaches the middle of the nanowire and electrons start to flow to the ground and d) The tip reaches the other side of the nanowire and more electrons flow through the nanowire to ground [4].

amount of energy needed to remove an electron from a solid to a point immediately outside the solid surface [7]. This means that it must be easier for the $\mathrm{ZnO}$ to have an electron taken from it than it is for the metal tip. If the reverse is true, an Ohmic contact is formed. When a Schottky barrier is formed, electrons flow from the nanowire to the external circuitry and this is exactly what must happen. When an Ohmic contact is formed between the nanowire and the tip, the electrons will keep flowing from the ground plate at a very slow rate and will not produce an electric potential. It is thus a necessity that a Schottky barrier should exist for the nanogenerator to work as desired.

To be more practical, the nanowires should be bent by something other than an AFM tip. The device that bends the nanowires should have a work function just above $4.5 \mathrm{eV}$ in order to form the Schottky barrier. Other than this restriction, the device can be anything. Making two sets of nanowires and coating the one set with gold, or anything else with a work function above $4.5 \mathrm{eV}$, then manufacturing a device from the two separate set of nanowires so that the nanowires are interposed as shown if Figure 4.9, will result in such a device. The upper set of nanowires replaces the function of the AFM tip in the above explanation of how the nanogenerator works.

If pressure is put onto any one of the two sides, top or bottom, the nanowires will touch each other and start to bend each other. This bending will then 


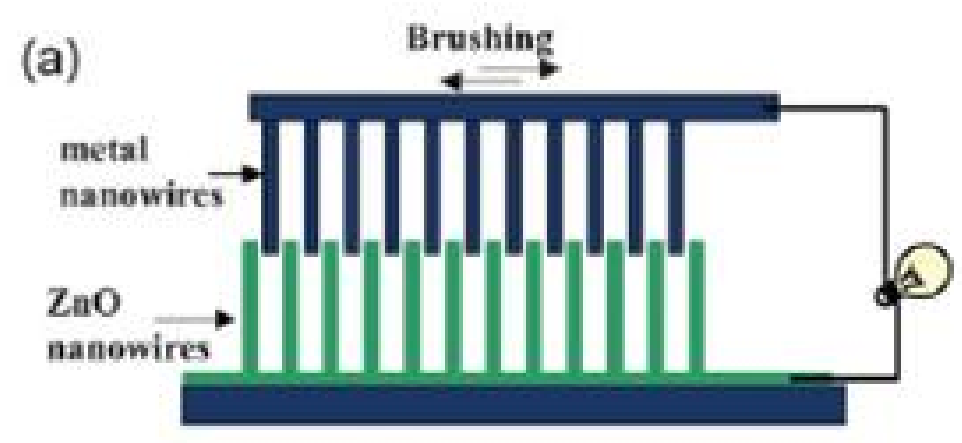

Figure 4.9: Two sets of nanowires brush against each other, thus creating a piezoelectric voltage. The top wires are coated with a metal to form a Schottky barrier between the bottom nanowires [7].

cause potential to build up due to the piezoelectric effect, and because the upper set of nanowires are of a metal with a work function high enough, a Schottky barrier will form and the charge-releasing process can be fulfilled. Because all the nanowires will not be of the same length, as illustrated in Figure 4.10, some nanowires will be too short to cause other nanowires to bend at certain times. The metal nanowires are normal $\mathrm{ZnO}$ nanowires covered in a layer of gold. The gold coated nanowires will bend the $\mathrm{ZnO}$ nanowires and act as the AFM tip. For simplicity, the gold coated nanowires are assumed to be stiffer than the $\mathrm{ZnO}$ nanowires and will thus not bend as much [8]. Piezoelectric potential is generated across the $\mathrm{ZnO}$ nanowires, with the stretched surface with positive potential, $V_{1}$, and the compressed surface with negative potential, $V_{2}$. The positive potential forms a reverse biased Schottky barrier with the gold coated nanowires and this will prevent the flow of current, while the negative potential forms a forward biased Schottky barrier that allows the flow of current from the $\mathrm{ZnO}$ nanowires through to the gold nanowires. When there are more than one set of nanowires in contact and forming a forward biased Schottky barrier, all the currents will add up. The voltage on the other hand is defined by the characteristics of one nanowire and does not add up like the current [8]. The voltage further depends on the system capacitance and the contact between the $\mathrm{ZnO}$ nanowires and gold coated nanowires.

If the assumption is made that $\mathrm{ZnO}$ is nonconductive, which is not the case, the output voltage can be a fraction of a volt [62]. From experiments, as 


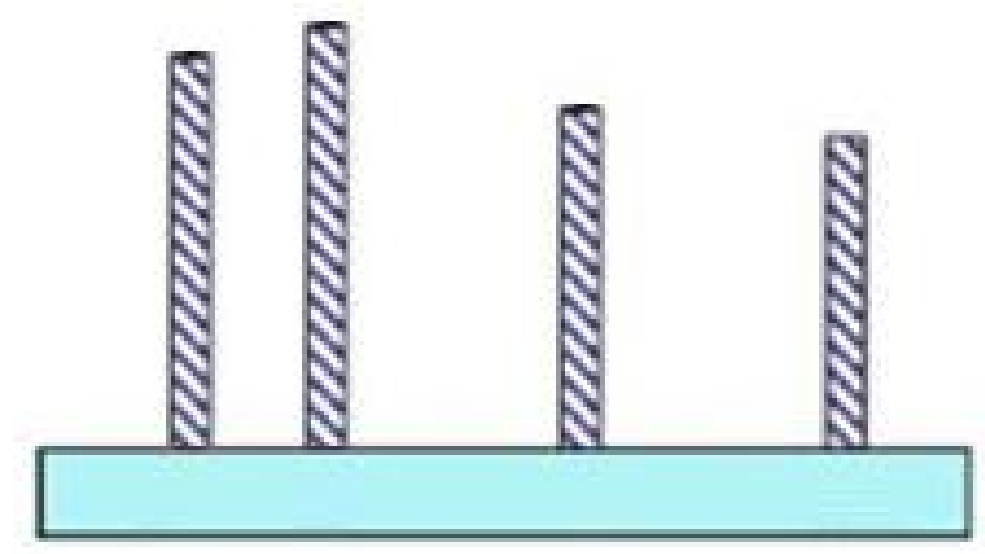

Figure 4.10: Graphical representation of nanowires of different lengths [8].

shown in Chapter 10, the output voltage is much lower than this, only a few millivolts, and this is because $\mathrm{ZnO}$ is conductive with high resistance. Figure 4.11 [9] shows the three possible states that a nanowire can be in. Nanowire I and II are in contact and will generate a voltage, nanowire III is in contact, but will not generate any voltage, and nanowire IV is not in contact. The two nanowires that are in contact, I and II, have an equivalent circuit model as shown in Figure 4.11 [9]. The circuit consists of a capacitor $\left(c_{o}\right)$, which represents the capacitance between the bottom and top nanowires, a resistance $\left(r_{c}\right)$ representing the contact resistance and resistance $r_{o}$, which represents the inner resistance of the nanowire [8]. Voltage $v_{0}$ represents the voltage created in the nanowire due to the piezoelectric effect and the diode represents the Schottky barrier that forms between the $\mathrm{ZnO}$ nanowires and the gold coated nanowires [9]. The circuit diagram for nanowire III, where there is no voltage generation, is shown in the next part of Figure 4.11. The nanowires just make contact with each other but do not generate a voltage. The model thus looks the same as those for nanowires I and II, except for the voltage source that is not present. The capacitors and resistance stay intact, only the voltage source is missing, as compared with the model for the first two nanowires. The last configuration is for nanowire IV, where the nanowire does not make any contact with the upper nanowire, as shown in Figure 4.11 [9]. It is clear that both the contact capacitance, as well as the contact resistance, will be omitted from the model, because there is no contact between the nanowires. It is further also clear that there is no voltage present, because the nanowire 
does not undergo any bending. One last capacitance is necessary to define for the system capacitance, as well as the capacitance between the two sets of nanowires (top and bottom pair), and this is represented by $c_{s}$ in Figure 4.11 [9].

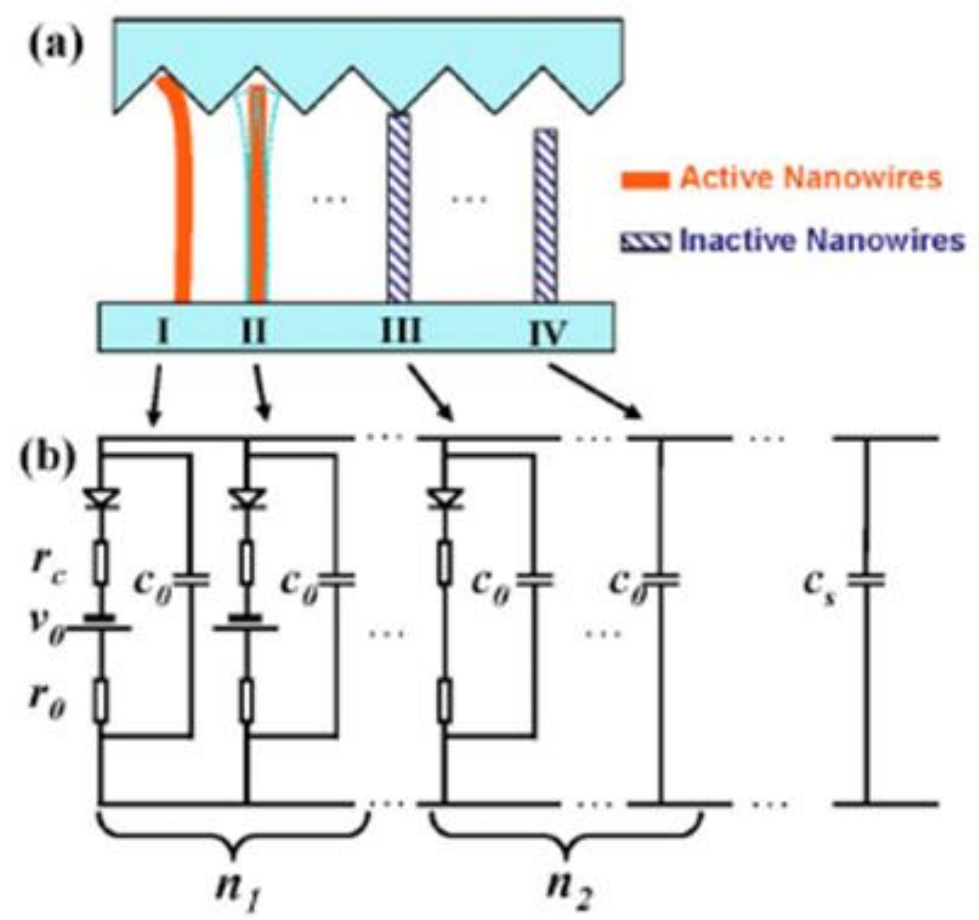

Figure 4.11: a) Nanowires of different length and their position relative to top electrode b) Circuit diagram of nanogenerator with nanowires in all possible configurations [9].

To calculate the voltage that is generated from the system it is assumed that the variables with the same symbols are equal, ie. all the capacitance values are equal. In practice each nanowire contact will have different values for resistance and capacitance, but for simplicity this is ignored. To further simplify the calculation, the system is approximated as if it is in quasistatic equilibrium. The voltage generated by a single nanowire equals [9]

$$
v_{o}=\frac{q_{o}}{c_{0}}
$$


If there are $n$ nanowires in contact at any given time and the system capacitance, $c_{s}$, is ignored, the system voltage is given by [9]

$$
v_{o}=\frac{n q_{o}}{n c_{o}}=\frac{q_{o}}{c_{o}} .
$$

This shows, as discussed earlier, that the maximum voltage output is due only to the maximum voltage of one nanowire [31]. The current is given by [9]

$$
I_{S}=\frac{n v_{S}}{\left(r_{o}+r_{c}\right)},
$$

where $v_{S}$ is the system voltage and $n$ is the number of active nanowires (nanowires that are in contact with other nanowires and generate a voltage). This shows that the current is the sum of all the currents in each individual nanowire [9]. This is only an approximation of the output voltage and output current. In practice other factors must be taken into account, but this gives a good approximation. From the above discussion it is easy to find methods to increase the performance of the system, including the following:

- Increase the number of active nanowires,

- increase in voltage will contribute to an increase in current,

- increase the degree of bending, and

- decrease the contact resistance.

Some of these methods are easier to achieve than others. The first three are not too hard to realise. The increase in the number of active nanowires can be done during the growth process, and certain steps can be made to try and grow nanowires of equal length, and this will be discussed in Chapter 6. Because the voltage and current are dependant on each other, a rise in one will ensure a rise in the other. The degree of bending is easy to increase in testing, but in practice this in not under the operator's direct control. To decrease the resistance is not as easy, this resistance comes from the contact of the two sets of nanowires and while some steps can be taken to make a better contact, this is rather difficult and is beyond the scope of this project. 


\subsubsection{Mathematics of piezoelectric nanowire}

To fully understand the phenomenon of piezoelectricity a look at the underlying mathematics is vital. Considering that a comprehensive mathematical treatment is beyond the scope of this project, only the basic formulation will be discussed.

The piezoelectric effect is only visible when the nanowire bends, in other words, when it is under strain or stress. The constitutive equation that describes the relationship between stress and strain is known as Hooke's law, and is given by

$$
S=s \times T \text {. }
$$

This means Strain $=$ Compliance $\times$ Stress [63]. Piezoelectricity has electrical properties as well, which are is given by the constitutive equation for common dielectrics,

$$
D=\varepsilon \times E,
$$

which translates to Charge Density $=$ Permittivity $x$ Electric Field [63]. Piezoelectricity is described by a combination of (4.4.4) and (4.4.5). When a strain or stress is applied to a material, an electric field is created, which in turn changes the charge density. These two equations must thus be combined for piezoelectricity, which is done as follows:

$$
\begin{aligned}
& S=s_{E} \times T+d^{t} \times E \\
& D=d \times T+\varepsilon_{T} \times E .
\end{aligned}
$$

The piezoelectric coupling terms are in the matrix $d$ [63]. Equations (4.4.6) and (4.4.7) are written in Strain-Charge form, but can also be rewritten in other forms if desired. Equations (4.4.6) and (4.4.7) give the basic equations for piezoelectricity, but to keep the notation the same, the equations are altered [62] to give

$$
\sigma_{P}=c_{p q} \times \varepsilon_{q}-e_{k p} \times E_{k}
$$




$$
D_{i}=e_{i q} \times \varepsilon_{q}+\kappa_{i k} \times E_{k},
$$

where $\sigma_{P}$ is the stress tensor, which is related to strain $\varepsilon_{q}$, the electric field $E$ and the electric displacement $D, c_{p q}$ is the linear elastic constant, $e_{k p}$ is the linear piezoelectric coefficient and $\kappa_{i k}$ is the dielectric constant [62]. Equations (4.4.8) and (4.4.9) are in exactly the same form as (4.4.6) and (4.4.7), the latter being only more defined than the former. Three more equations are used to solve piezoelectric related problems. The first is the mechanical equilibrium condition, when there is no body force acting on the nanowire [62],

$$
\nabla \cdot \sigma=0
$$

The compatibility equation is a geometrical constraint that must be satisfied [62],

$$
e_{i l m} e_{j p q} \frac{\partial^{2} \varepsilon_{m p}}{\left(\partial x_{l} \partial x_{q}\right)}=0 .
$$

The final equation assumes that there is no free charge $\rho_{e}^{b}$ in the nanowire. In other words, the Gauss equation

$$
\nabla \cdot \vec{D}=\rho_{e}^{b}=0
$$

must be satisfied [62]. The matrices in (4.4.8) and (4.4.9) can be written in a certain pattern, because of the wurtzite structure of $\mathrm{ZnO}$ [62]:

$$
\begin{gathered}
c_{p q}=\left(\begin{array}{cccccc}
c_{11} & c_{12} & c_{13} & 0 & 0 & 0 \\
c_{12} & c_{11} & c_{13} & 0 & 0 & 0 \\
c_{13} & c_{13} & c_{33} & 0 & 0 & 0 \\
0 & 0 & 0 & c_{44} & 0 & 0 \\
0 & 0 & 0 & 0 & c_{44} & 0 \\
0 & 0 & 0 & 0 & 0 & \frac{\left(c_{11}-c_{12}\right)}{2}
\end{array}\right) \\
e_{k p}=\left(\begin{array}{cccccc}
0 & 0 & 0 & 0 & e_{15} & 0 \\
0 & 0 & 0 & e_{15} & 0 & 0 \\
e_{31} & e_{31} & e_{33} & 0 & 0 & 0
\end{array}\right)
\end{gathered}
$$




$$
\kappa_{i k}=\left(\begin{array}{ccc}
\kappa_{11} & 0 & 0 \\
0 & \kappa_{11} & 0 \\
0 & 0 & \kappa_{33}
\end{array}\right) .
$$

This simplifies calculations to some degree. Equations (4.4.8) to (4.4.12), along with appropriate boundary conditions, fully describe a static piezoelectric system, but the solutions of these equations are complex and an analytical solution does not always exist [62]. In order to approximate a solution, a perturbation expansion of the linear equations is needed in order to simplify the analytical solution [62]. A perturbation expansion is used when an equation cannot be solved exactly and it is then converted by computing a power series expansion in a small parameter. The first few terms then give a good approximation, for example [64]

$$
T(\alpha)=T_{0}+\alpha T_{1}+\alpha^{2} T_{2}+\ldots
$$

The equation $T(\alpha)$ is expanded by a power series and then, using only a few terms, a good approximation can be made [64]. To derive the piezoelectric potential distributed in the nanowire, different orders of the electromechnical coupled effect must be viewed [62]. A perturbation parameter, $\lambda$, is introduced and is defined as $e_{k p}^{\prime}=\lambda e_{k p}$, which is introduced to trace the magnitudes of contributions made by different orders of the electromechanical effect to the total piezoelectric potential [62]. When $\lambda=1$ the exact same system, as described above, is realized, and when $\lambda=0$, no electromechanical coupling is present [62]. Looking only at the first three orders of perturbation, which relate to bending of the nanowire without piezoelectric effect, bending of the nanowire which generates a potential and, lastly, bending of the nanowire which causes a potential which potential causes bending. By substituting the perturbation parameter into (4.4.8) and (4.4.9) they become:

Zeroth order:

$$
\begin{aligned}
\sigma_{P}^{(0)} & =c_{p q} \varepsilon_{q}^{(0)} \\
D_{i}^{(0)} & =\kappa_{i k} E_{k}^{(0)}
\end{aligned}
$$

First order:

$$
\begin{aligned}
& \sigma_{P}^{(1)}=c_{p q} \varepsilon_{q}^{(1)}-e_{k p} E_{k}^{(0)} \\
& D_{i}^{(1)}=e_{k p} \varepsilon_{q}^{(0)}+\kappa_{i k} E_{k}^{(1)}
\end{aligned}
$$


Second order:

$$
\begin{gathered}
\sigma_{P}^{(2)}=c_{p q} \varepsilon_{q}^{(2)}-e_{k p} E_{k}^{(1)} \\
D_{i}^{(2)}=e_{k p} \varepsilon_{q}^{(1)}+\kappa_{i k} E_{k}^{(2)} .
\end{gathered}
$$

Using these equations, instead of (4.4.8) and (4.4.9), makes the calculations a little easier. As stated above, the zeroth order is that in which the nanowire bends but the piezoelectric effect is not present, which in turn means there is no electric field [62]. This simplifies the equations even more, because $E_{k}^{(0)}=0, D_{i}^{(0)}=0$. Substituting these two conditions into the other two equations gives $\sigma_{P}^{(1)}=0, \varepsilon_{p}^{(1)}=0, D_{p}^{(2)}=0$ and $E_{p}^{(2)}=0$. Substituting this back into the original equations yields [62]

Zeroth order:

$$
\sigma_{P}^{(0)}=c_{p q} \varepsilon_{q}^{(0)}
$$

First order:

$$
D_{i}^{(1)}=e_{k p} \varepsilon_{q}^{(0)}+\kappa_{i k} E_{k}^{(1)}
$$

Second order:

$$
\sigma_{P}^{(2)}=c_{p q} \varepsilon_{q}^{(2)}-e_{k p} E_{k}^{(1)}
$$

The physical meaning of these equations is easy to understand. Under the different approximations these equations correspond to the decoupling and coupling of the electric field and mechanical deformation. The zeroth order is a result of mechanical deformation only, and no piezoelectric effect is observed. The first order is a result of the piezoelectric effect; voltage due to bending of the nanowire. The second order is a result of the first feedback loop; voltage generated due to bending and the voltage causes more bending [62]. In this thesis only the first order approximation is of interest. The zeroth order can be ignored, because the piezoelectric effect is observed when bending the nanowire. The second order can also be ignored, because the resulting voltage is too small to cause any sufficient bending of the nanowire.

To further simplify the solution we assume the nanowire has a cylindrical shape with a uniform cross section, a diameter of $2 a$ and a length of $l$ [62]. The shape of the nanowire is not cylindrical, but the assumption makes the calculations easier. The true form of the nanowire will be discussed in Chapter 5. To even further simplify things, the Young modulus, $E$, and Poisson ratio, $v$, are also approximated [62]. The Young modulus gives an indication of the stiffness of the nanowire and the Poisson ratio is the ratio between the 
contraction and extension of the nanowire. For convenience $a_{p q}$ is defined as the inverse matrix $c_{p q}$. The strain and stress relation is then given by [62]

$$
\begin{gathered}
\quad\left(\begin{array}{c}
\varepsilon_{x x}^{(0)} \\
\varepsilon_{y y}^{(0)} \\
\varepsilon_{z z}^{(0)} \\
2 \varepsilon_{y z}^{(0)} \\
2 \varepsilon_{z x}^{(0)} \\
2 \varepsilon_{x y}^{(0)}
\end{array}\right)=\Sigma_{q} a_{p q}^{\text {isotropic }_{\sigma_{q}}^{(0)}}= \\
\frac{1}{E}\left(\begin{array}{cccccc}
1 & -v & -v & 0 & 0 & 0 \\
-v & 1 & -v & 0 & 0 & 0 \\
-v & -v & 1 & 0 & 0 & 0 \\
0 & 0 & 0 & 2(1+v) & 0 & 0 \\
0 & 0 & 0 & 0 & 2(1+v) & 0 \\
0 & 0 & 0 & 0 & 0 & 2(1+v)
\end{array}\right)\left(\begin{array}{c}
\sigma_{x x}^{(0)} \\
\sigma_{y y}^{(0)} \\
\sigma_{z z}^{(0)} \\
\sigma_{y z}^{(0)} \\
\sigma_{z x}^{(0)} \\
\sigma_{x y}^{(0)}
\end{array}\right)
\end{gathered}
$$

The configuration of the nanowire is such that one end is affixed on a substrate, while the other end is free and is pushed by a lateral force $f_{y}$. The Saint-Venant theory of bending is applied, which states that if calculations are made far enough away from the fixed end, the solution will be uniform across the shape. The stress induced in the nanowire is given [62] by

$$
\begin{gathered}
\sigma_{z x}^{(0)}=-\frac{f_{y}}{4 I_{x x}} \frac{1+2 v}{1+v} x y \\
\sigma_{y z}^{(0)}=-\frac{f_{y}}{I_{x x}} \frac{3+2 v}{8(1+v)}\left[a^{2}-y^{2}-\frac{1-2 v}{3+2 v} x^{2}\right] \\
\sigma_{z z}^{(0)}=-\frac{f_{y}}{I_{x x}} y(l-z) \\
\sigma_{x x}^{(0)}=\sigma_{x y}^{(0)}=\sigma_{y y}^{(0)}=0
\end{gathered}
$$

where

$$
I_{x x}=I_{y y}=\frac{\pi}{4} a^{2}
$$

These equations are only valid in regions far away from the fixed end, as mentioned above. Regions twice the diameter of the nanowire away will be 
far enough. A remnant displacement, body charge and surface charge are defined as [62]

$$
\begin{aligned}
& \overrightarrow{D^{R}}=e_{k p} \varepsilon_{q}^{(0)} \hat{i_{k}} \\
& \rho^{R}=\nabla \cdot \overrightarrow{D^{R}}
\end{aligned}
$$

and

$$
\Sigma^{R}=\vec{n} \cdot \overrightarrow{D^{R}}
$$

respectively.

The remnant displacement can be transformed into an elementary electrostatic problem, using the Poisson equation [62], as

$$
\nabla \cdot\left(\kappa_{i k} E_{k}^{(1)} \overrightarrow{i_{i}}=\rho^{R}\right.
$$

Equations (4.4.32) to (4.4.35) can be rewritten as [62]

$$
\rho^{R}=\frac{f^{y}}{I_{x x} E}\left[2(1+v) e_{15}+2 v e_{31}-e_{33}\right] y
$$

and

$$
\Sigma^{R}=0
$$

These equations are independent of the vertical height, thus the electric potential is independent of the vertical height. This means that the nanowire is approximately like a parallel plate capacitor. The electric potential is then given by [62]

$$
\varphi=\frac{1}{8 \kappa_{11}} \frac{f_{y}}{I_{x x} E}\left[2(1+v) e_{15}+2 v e_{31}-e_{33}\right]\left[\frac{\kappa_{0}+3 \kappa_{11}}{\kappa_{0}+\kappa_{11}} \frac{r}{a}-\frac{r^{3}}{a^{3}}\right] a^{3} \sin \theta
$$

The solution presented here is within 6 percent of simulated values [62].

The mathematics presented here was presented in [62]. Considerable time and effort was put into understanding each individual equation, but because many intermediary steps were omitted, it was not an easy task. The basic idea behind the mathematics is easy to grasp. The aim of this thesis was not 
to develop and understand the mathematics behind the voltage generation process caused by piezoelectric effect, but rather to utilize this concept to design and manufacture a pressure sensor. The mathematics was included, because some of the equations shed some light on how the process works. The final answer also gives an indication as to where one can tweak to get maximum voltage out of the nanowire. Throughout the mathematics, certain approximations and assumptions are made. In practice the approximations and assumptions are not valid, and a big difference in the output voltage can thus be observed. Derivation of the theoretical mathematical model, which include all variables, will be near impossible. 


\section{Chapter 5}

\section{Nanostructures}

\subsection{What are nanostructures?}

Everyday devices are becoming increasingly smaller and smaller. Intel is already preparing to make processors with $45 \mathrm{~nm}$ to $32 \mathrm{~nm}$ memory cells, the smallest to date [65]. Satisfying the increasing demand to make products smaller will become easier with nanotechnology. Nanostructures will be used to make novel devices that operate on the nanoscale. Nanostructures are defined as any structure that has at least one dimension smaller than $100 \mathrm{~nm}$. Nanostructures can be viewed as falling into three categories: one-dimensional structures like thin films with a thickness of a few nanometers [66]; two-dimensional structures like tubes or wires that have a diameter less than a $100 \mathrm{~nm}$ and an arbitrary length; and three-dimensional structures like nanoparticles with all three dimensions less than $100 \mathrm{~nm}$ [67]. Onedimensional structures are used primarily for thin films which have many applications in solar cells or the coating of glass or lenses [66]. Two-dimensional structures have many applications, ranging from pressure sensors to gas sensors and many more. Three-dimensional structures are used in medical applications to target specific cells [67]. Carbon nanotubes (CNT) are one of the most studied nanostructures. CNT has found a wide range of applications ranging from structural [68], electric circuits [69], paper batteries [70], vessels for drug delivery, solar cells [71], ultracapacitors [72] and many more. As experiments with CNT evolve, new uses will be found and it will improve current technology. CNT is just one of the nanostructures being used and studied today, which on its own has already made a huge impact on every- 
day products. For this project two-dimensional structures were used in the form of zinc oxide nanowires. Zinc oxide was chosen because of the piezoelectric effect that it exhibits due to its unique crystal structure. There are many different methods to grow these nanowires, each with its advantages and disadvantages. A multitude of applications for these nanowires already exist. These points will be discussed in more detail in the chapter that follows.

\subsection{Crystal Structure}

Crystal structure plays a big role in the properties of a material. Carbon comes in a variety of different forms, carbon-60, which can be a round ball, or buckyball, or a long cylinder, a carbon nanotube [73].

Zinc oxide nanowires also have a unique crystal structure. Figure 5.1 shows the crystal structure of $\mathrm{ZnO}$ [4]. This crystal structure is not unique to $\mathrm{ZnO}$ nanowires, but to $\mathrm{ZnO}$ nanostructures as a whole. Zinc oxide crystallizes in three forms: Hexagonal wurtzite, cubic zincblende and cubic rocksalt, which is very rare. In both the wurtzite and zincblende the zinc and oxide have tetrahedral centres [74]. The $\mathrm{ZnO}$ structures used in this project have a wurtzite structure [4]. Wurtzite is a zinc iron sulphide mineral and its unique crystal structure was named the wurtzite crystal structure. It is a member of the hexagonal crystal system and consists of tetrahedral coordinated zinc and oxide atoms stacked in an ABABAB pattern [75]. As explained in Chapter 4, it is this crystal structure that gives $\mathrm{ZnO}$ its unique properties. When a force is applied to the $\mathrm{ZnO}$ nanowires the tetrahedral symmetry is broken, which causes a displacement of the atoms, which in turn creates a potential difference, or piezoelectric voltage.

A lot more can be said about the crystal structure in terms of crystal orientation, however, going into more detail is beyond the scope of this project. Only the pressure sensor application of $\mathrm{ZnO}$ nanowires was investigated and a more in depth analysis of the crystal structure would not have benefited this aim. 


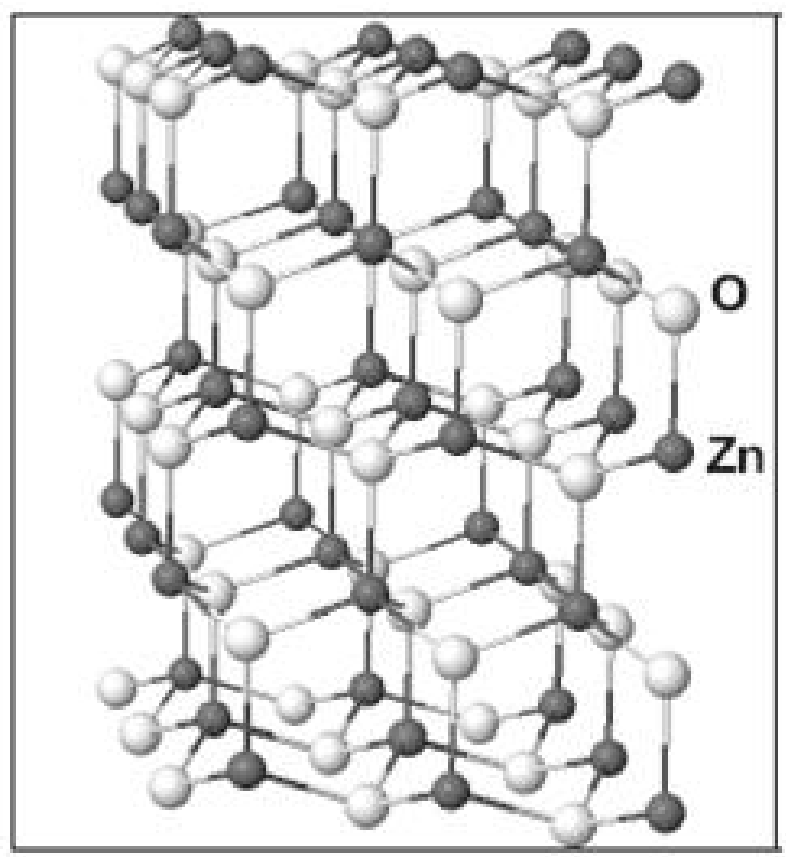

Figure 5.1: Crystal structure of $\mathrm{ZnO}$ [4]

\subsection{Methods of growing nanostructures}

Over the last couple of years nanotechnology has gained a lot of attention. A variety of different methods of growing different nanostructures has emerged from all this extra attention in the nanofield. Just looking at nanowires, a wide range of methods already exists, like the vapour liquid solid (VLS) growth process [76] [77] [11] [56] [78], laser-assisted catalytic growth [79], chemical vapor deposition [80], template assisted synthesis [81], electrospinning [13] and electrodeposition [82] . All of these methods have their advantages and disadvantages, some working better than others.

\subsubsection{Vapour Liquid Solid}

The vapour liquid solid (VLS) method is used to grow two-dimensional nanostructures. VLS is the method, of the few listed above, with the most literature available, which made it a good method to use in this project. The VLS method makes use of a tube inside an oven that can heat up to $1000^{\circ} \mathrm{C}$. Chemicals are placed inside the tube with a substrate placed a fixed distance from the chemicals. Argon gas is then made to flow through the system at a fixed 
rate. The chemicals and substrate are at different temperatures. The chemicals are at a higher temperature, which causes them to evaporate. The argon gas will then move this vapour to a cooler spot, the substrates, where it will act with a catalyst on the substrate and begin to grow nanostructures. The main problem with this method is to get all the right parameters. The three most important parameters are the distance between the chemicals and substrate, the temperature and the argon gas flow rate. If the substrate is too close to the chemicals, and thus at too hot a place, chemical vapours will not react with the catalyst but rather continue to evaporate. If it is too far, it would already have found a cooler place, and settled there before reaching the substrate. The temperature must be just right, first of all for the chemicals to evaporate and secondly the substrate should be cold enough for a reaction to take place. The speed at which the argon gas flows through the system is just as important: if it flows too fast, the vapour will move right over the sample and will not have enough time to react and if it is too slow, the vapour will not reach the sample at all. At first it is hard to get a complete setup correct, but once it is established it works very well. The other problem is the high temperature at which the system has to operate. Not many materials can handle such extreme heat.

VLS is one of the most studied methods of growing nanowires, with almost one million hits in a Google search [83]. Finding parameters for growing nanowires is thus not too difficult. However, everyone uses their own individual equipment, which will also influence the parameters. Even small changes in the temperature, pressure or moisture levels in the room can influence the growth of the nanowires. One of the biggest problems is dust or dirt that collects on the $\mathrm{Si} / \mathrm{Au}$ substrate. It is difficult to keep a sample clean throughout the entire process. When the heat inside the tube varies, a different nanostructure can form. When the source chemicals are $\mathrm{ZnO}$, the temperature plays a vital role. At $1400^{\circ} \mathrm{C}$ wool-like structures are formed, as seen in Figure 5.2 [51].

As the temperature rises, the wires get thicker. At very high temperatures the wires will change to nanobelts [51]. This shows how changing just one of the many parameters involved in the VLS growth method can result in the growth of different nanostructures. The VLS method can produce nanowires, nanotubes, nanowalls, nanosprirals, nanosprings, nanoribbons, nanobelts and many more [51]. All of these different structures can be grown 


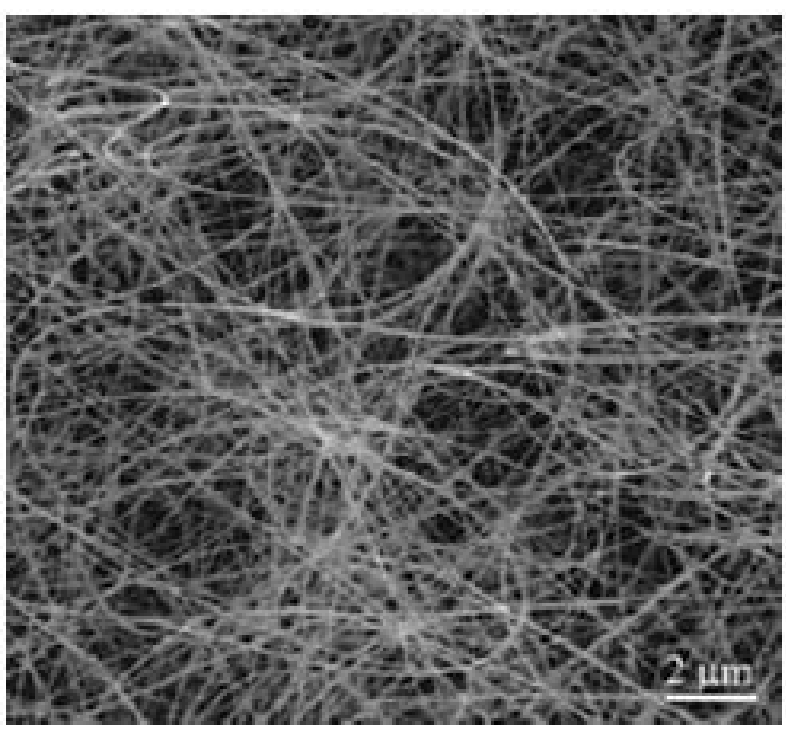

Figure 5.2: Wool-like $\mathrm{ZnO}$ structures [10]

by simply adjusting a few of the parameters involved in the VLS method. Temperature, as already mentioned, is one of the most crucial parameters when growing different structures.

The growth shown above was achieved without any catalyst and with only $\mathrm{ZnO}$ powder. When graphite is mixed with $\mathrm{ZnO}$ in a 1:1 ratio, the vaporization temperature of the powders will decrease from $1300^{\circ} \mathrm{C}$ to $900^{\circ} \mathrm{C}$ [84] [11]. The graphite and $\mathrm{ZnO}$ powders are mixed in a ceramic boat and placed inside a tube, inside an oven. The oven is then heated to above $900^{\circ} \mathrm{C}$, where the following reactions then take place [11]:

$$
\begin{gathered}
\mathrm{ZnO}(\mathrm{s})+\mathrm{C}(\mathrm{s}) \rightarrow \mathrm{Zn}(g)+\mathrm{CO}(g) \\
\mathrm{CO}(g)+\mathrm{ZnO}(s) \rightarrow \mathrm{CO}_{2}(g)+\mathrm{Zn}(g)
\end{gathered}
$$

Once the vapour reaches the Silicon sample that has a thin layer of gold on top of it, the following reactions take place [11]:

$$
\begin{gathered}
\mathrm{Zn}(g)+\mathrm{CO}(g) \rightarrow \mathrm{ZnO}(\mathrm{s})+\mathrm{C}(\mathrm{s}) \\
\mathrm{C}(\mathrm{s})+\mathrm{CO}_{2}(g) \rightarrow 2 \mathrm{CO}(g)
\end{gathered}
$$

These four chemical reactions describe the process from start to finish. 
Although the exact function of the graphite powder is not yet known, it is believed to reduce the vaporization temperature, but any other effects are still unclear [85].

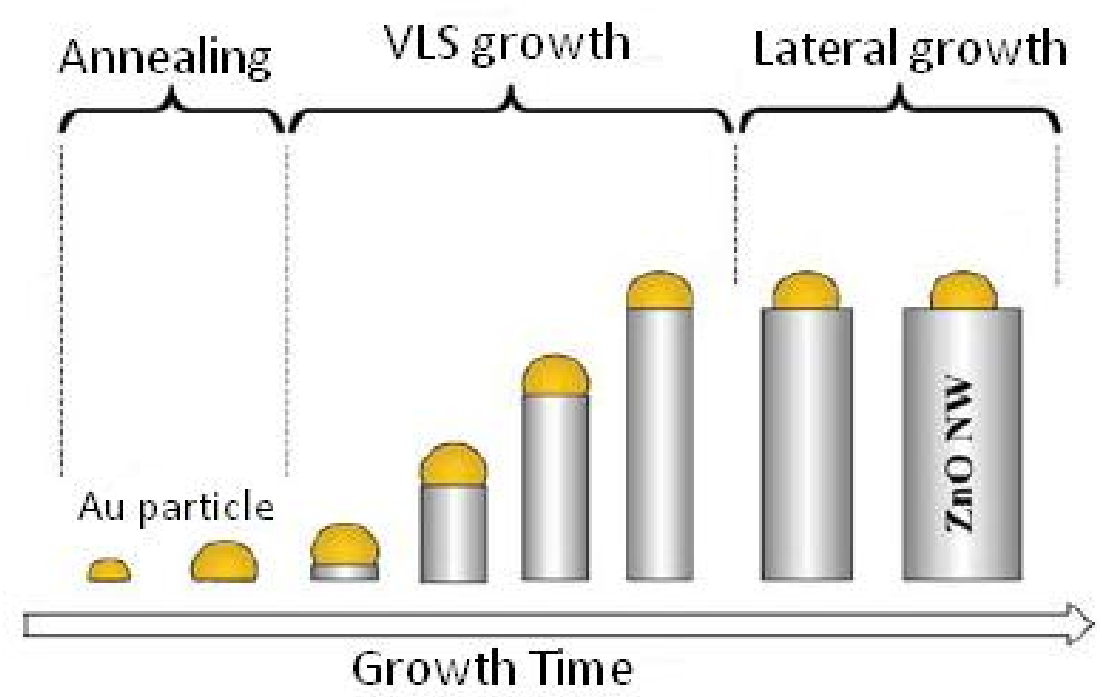

Figure 5.3: VLS growth visualized step by step [11].

Figure 5.3 shows the VLS growth process [85]. The silicon substrate is coated with a thin film of gold, $20 \mathrm{~nm}$ thick. The sample is then loaded into the furnace to anneal the gold. Annealing is the process of heating up a material by which some of the material's properties are changed. In the case of the thin gold film, the heating causes small, nanoparticles sized gold droplets to form, as seen in the first two pictures in Figure 5.3. After annealing, the furnace is heated up to above $900^{\circ} \mathrm{C}$, where the $\mathrm{ZnO}$ and graphite powders will evaporate. Argon gas then carries the vapour to the silicon substrate with gold nanoparticles, the $\mathrm{ZnO}$ powders start to form the nanowire underneath a gold nanoparticle. This process keeps going up to a certain point, where vertical growth stops and the nanowires' diameter starts to expand [85]. The exact process at work during the growth of the nanowire underneath the gold nanoparticle is still not understood [85] and also hard to determine, because of the high temperature at which the growth takes place.

From the above model it is easy to see what parameters will affect the size of the nanowires. The first obvious parameter is the thickness of the 
gold layer. The thicker the layer, the larger the gold droplet will be and the larger the nanowire diameter will be. The other obvious parameter is the growth time. At a certain point the vertical growth stops and only the diameter continues to expand. Stopping the process at that exact point will yield the longest and thinnest nanowires. Advantages of the VLS method are that it is very easy to implement and there is an abundance of material available. Disadvantages include the need of a catalyst, like gold, which is expensive, and the high operating temperatures.

The growth setup for this project was as follows:

1. Graphite powder and $\mathrm{ZnO}$ powder were mixed in a 1:1 ratio.

2. The powders were placed in a ceramic boat and loaded into the centre of the furnace.

3. Silicon samples with a $20 \mathrm{~nm}$ layer of gold were then loaded into the furnace, between $50 \mathrm{~mm}$ to $200 \mathrm{~mm}$ away from the ceramic boat.

4. The furnace was heated to $500^{\circ} \mathrm{C}$ to anneal the gold layer.

5. The furnace was then heated up to $920^{\circ} \mathrm{C}$ for growth.

6. From start to finish argon gas was flowing at a rate of 60 standard cubic centimeter per minute (sccm).

The gold layers were deposited using a high voltage sputter unit. The thickness of the layer is determined by the sputter time and sputter voltage. The thicker the gold layer, the larger the diameter of the grown nanowires will be [11].

The Si/Au-samples are placed a certain distance from the source boat, because a temperature difference between the two is essential. The temperature drops away from the centre point of the furnace. Table 5.1 shows how the temperature drops inside the furnace, from the centre at $920^{\circ} \mathrm{C}$ to $644^{\circ} \mathrm{C}$ $200 \mathrm{~mm}$ away from the centre.

Figure 5.4 shows a picture taken by the SEM of the $\mathrm{ZnO}$ nanowires grown by the VLS method. Figure 5.4 was taken with low magnification and shows how the nanowires are aligned. Figure 5.5 was taken with high magnification in order to see individual nanowires.

Results obtained with the AFM and light microscope are shown in Appendix A. The figures each represent a different set of nanowires that was 
Table 5.1: Temperature profile of the furnace ( $0 \mathrm{~mm}$ is in the centre of the furnace).

\begin{tabular}{|c|c|}
\hline Temperatue $\left({ }^{\circ} \mathbf{C}\right)$ & Distance from center $(\mathbf{m m})$ \\
\hline 920 & 0 \\
\hline 901 & 10 \\
\hline 874 & 30 \\
\hline 855 & 50 \\
\hline 828 & 80 \\
\hline 791 & 100 \\
\hline 763 & 120 \\
\hline 717 & 150 \\
\hline 644 & 200 \\
\hline 533 & 250 \\
\hline 395 & 300 \\
\hline 330 & 350 \\
\hline 200 & 400 \\
\hline
\end{tabular}

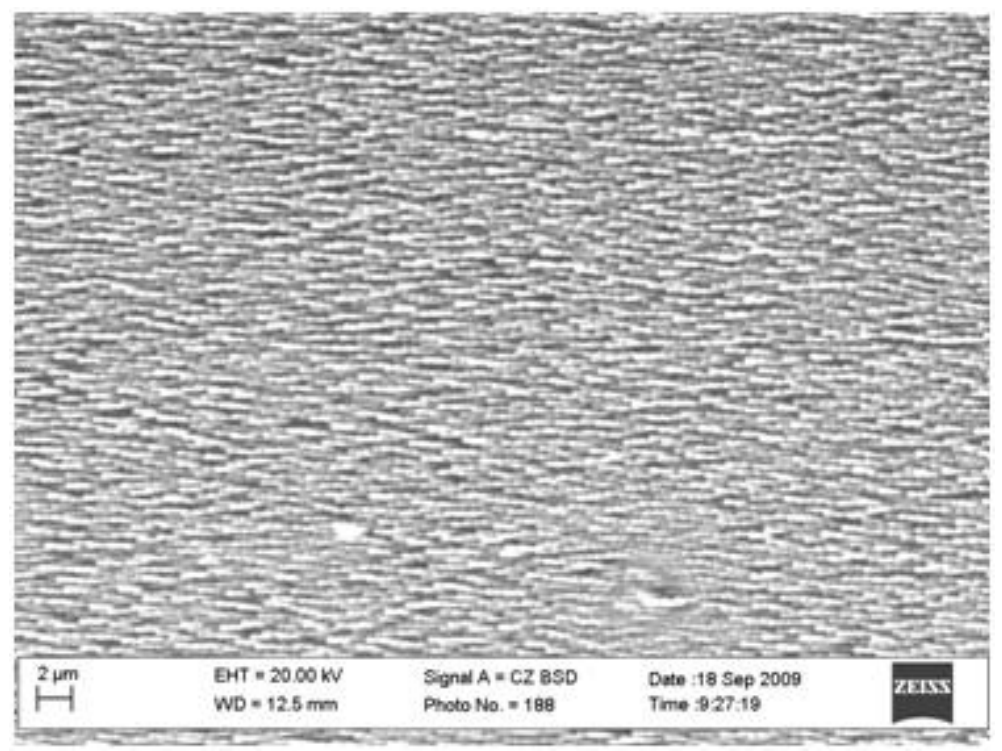

Figure 5.4: Picture of VLS growth as taken by SEM. Picture was taken with low magnification of only 1000 . 


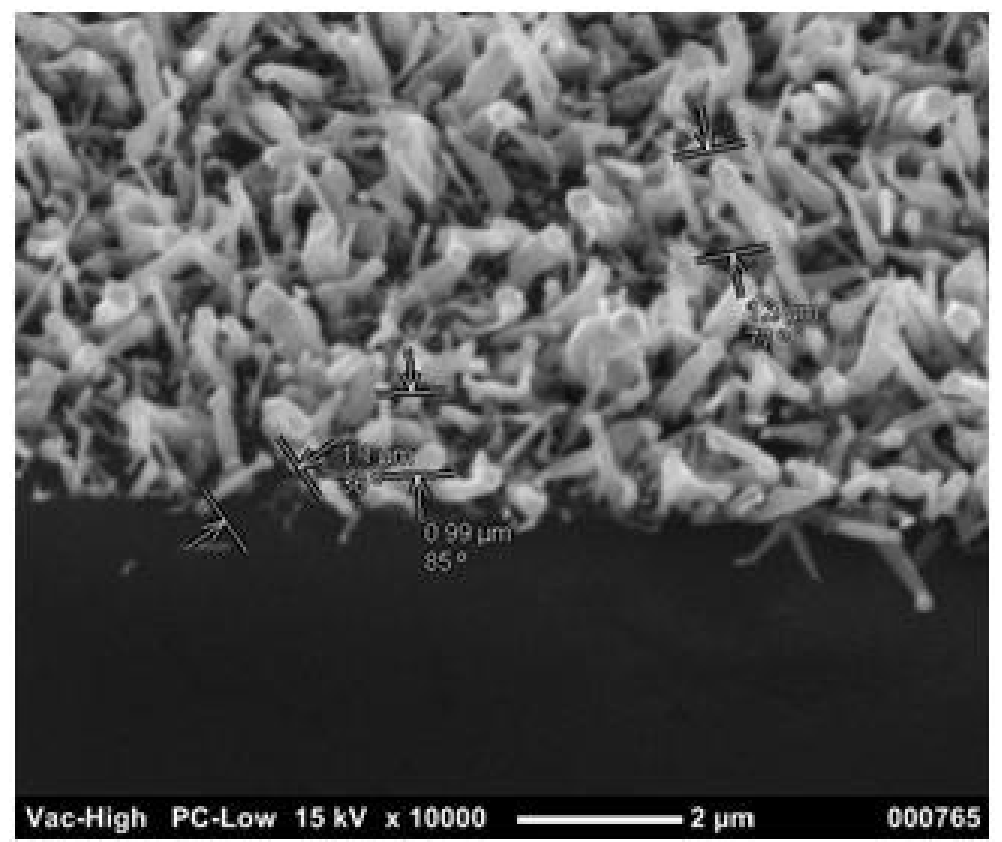

Figure 5.5: Picture of VLS growth as taken by SEM. Picture was taken with high magnification.

grown, with at least one parameter different from those of the other sets of nanowires. It is not always very clear what influence each parameter has sometimes it can appear as if it had no effect on the growth. However, looking at the figures in Appendix A, some subtle differences in the nanowires are visible.

\subsubsection{Laser Assisted Catalytic Growth}

Looking at each method separately, laser-assisted catalytic growth, as implied by the name, requires a laser to assist with the growth. A catalyst should also be present for growth. A pulsed laser is used to vaporize a solid target containing the desired material and catalyst, the resulting liquid nanocluster material is at an elevated temperature which directs the growth and also defines the diameter of the nanowires [79]. It is easy to change the diameter of the nanowires by changing only the intensity of the laser. The drawback to this method is that a laser is required and the exact growth mechanism is not yet fully understood. The requirement for a catalyst also makes this method a bit pricier to perform. The only laser suitable for laser-assisted 
catalytic growth at Stellenbosch University is at the Physics Department and the method was not considered, due to the fact that the laser is in high use most of the time. Another reason why this method was not used was because of limited knowledge of the field and the scarcity of references on this method. The advantages of this method is that it would be less expenisve to produce nanowires, once the initial cost of the laser is dissipated. It is also quicker than the VLS method.

\subsubsection{Chemical Vapour Deposition}

Chemical vapour deposition (CVD), as the name implies, is a chemical process to produce solid materials. These materials are of high purity and high performance. In the CVD process a substrate is exposed to one or more vaporizing material that acts as a precursor which reacts on the surface of the substrate to produce the desired deposition [80]. The substrate is usually covered with a catalyst to speed up the process. The CVD method requires a lot of background knowledge in chemistry to fully understand the growth mechanism. Although the author did not have the necessary chemical background, this method was tried to some extent to grow $\mathrm{ZnO}$ nanowires.

The CVD method used is a variation on normal CVD processes, because it uses a liquid solution as a chemical base. The chemicals are dissolved in de-ionized water and other liquids and then the substrate is placed upside down in the liquid, because the area on which the growth will take place must be in contact with the liquid. The substrate is then placed inside an oven and heated up. The properties of the chemicals in the solution change as the temperature increases. The chemicals start to evaporate and then collect on the substrate that was placed on top of the liquid. By pre-treating the substrate with an appropriate catalyst, nanostructures can grow on the substrate. This method also makes use of hydrolysis, a chemical process in which water molecules are split into hydrogen and hydroxide ions [86].

Aqueous solution growth of nanostructures is advantageous compared to other methods, due to the low growth temperature [87]. By changing the thickness of the catalyst layer, the thickness of the nanowires can be controlled. The main disadvantage, as mentioned above, is that the method relies a lot on a basic knowledge of chemistry.

For this project, $\mathrm{ZnO}$ nanowires were grown from an aqueous solution containing a Zn-salt, such as zinc acetate dehydrate or zinc nitrate hexahy- 
drate. Other materials were also used for the growth of the nanowires, such as HMTA (methamine), but the exact function of the HMTA is not fully understood [88] and will thus be omitted from the discussion.

For the catalyst a thin film was deposited onto the silicon substrate via the spin table. The thickness of the layer can be controlled by adjusting the spinning rate and also the spinning time. The chemicals used for the catalyst were zinc acetate dehydrate ( $\mathrm{Zn} \mathrm{Ac}$ ) dissolved in ethanol [88]. The solution was prepared to make a $5 \mathrm{mM}$ solution. The molar mass of $\mathrm{Zn}$ Ac was calculated as $219.5276 \mathrm{~g} / \mathrm{mol}$, and for making a $5 \mathrm{mM}$ (millimol/L) solution the amount of $\mathrm{Zn} \mathrm{Ac}$ in grams per liter can be calculated. This solution was spin-coated onto the substrate and then placed in an oven to evaporate the liquid and leave only small particles of $\mathrm{Zn}$ Ac that would act as a catalyst. The temperature of the oven was just above the decomposition temperature of $\mathrm{Zn} \mathrm{Ac}\left(250^{\circ} \mathrm{C}\right.$ [88]). This process was repeated until a uniform layer was formed. The seed layer is critical in determining the diameter of the grown nanowires. Just as in the case of VLS with the gold layer, the thickness of the layer determines the diameter of the nanowires. The diameters of the nanowires can be adjusted by adjusting the thickness of the seed layer. The thickness of the seed layer can be adjusted, as mentioned above, by changing the spinning parameters, as well as by changing the amount of $\mathrm{Zn} \mathrm{Ac}$ in the solution.

The next step is to grow the nanowires. For this step, zinc nitrate hexahydrate and HMTA were dissolved in DI water. The concentration of both was $1 \mathrm{M}$ [88]. Once again, the molar mass of both was determined as 297.51048 $\mathrm{g} / \mathrm{mol}$ and $140.18628 \mathrm{~g} / \mathrm{mol}$ respectively, and from that the gram per liter of each chemical can be calculated. The pretreated substrate was then placed in the solution, with the pretreated side facing down, on top of the liquid solution. The solution was then heated to just under $100^{\circ} \mathrm{C}$, to prevent the water from evaporating, and left for three hours for the growth to take place.

The chemical process can be explained by the following equations [88]:

$$
\begin{gathered}
\mathrm{C}_{6} \mathrm{H}_{1} 2 \mathrm{~N}_{4}+6 \mathrm{H}_{2} \mathrm{O} \rightarrow 6 \mathrm{CH}_{2} \mathrm{O}+4 \mathrm{NH}_{3} \\
\mathrm{NH}_{3}+\mathrm{H}_{2} \mathrm{O} \rightarrow \mathrm{NH}_{4}^{+} \mathrm{OH}^{-} \\
\mathrm{Zn}\left(\mathrm{NO}_{3}\right)_{2} \rightarrow \mathrm{Zn}^{2+}+2 \mathrm{NO}_{3}^{-}
\end{gathered}
$$




$$
\begin{gathered}
\mathrm{Zn}^{2+}+4 \mathrm{NH}_{3} \rightarrow \mathrm{Zn}\left(\mathrm{NH}_{3}\right)_{4}^{2+} \\
\mathrm{Zn}^{2+}+4 \mathrm{OH}^{-} \rightarrow \mathrm{Zn}(\mathrm{OH})_{4}^{2-} \\
\mathrm{Zn}\left(\mathrm{NH}_{3}\right)_{4}^{2+}+2 \mathrm{OH}^{-} \rightarrow \mathrm{ZnO}+4 \mathrm{NH}_{3}+\mathrm{H}_{2} \mathrm{O} \\
\mathrm{Zn}(\mathrm{OH})_{4}^{2-} \rightarrow \mathrm{ZnO}+\mathrm{H}_{2} \mathrm{O}+2 \mathrm{OH}^{-}
\end{gathered}
$$

The HMTA provides the hydroxide ions and the ammonia molecules to the solution. The zinc nitrate hexahydrate acts as the precursor for $\mathrm{ZnO}$. After a while the $\mathrm{pH}$-value of the solution will change due to the ammonia that is present in the water and the solution will turn white. The solution will then turn transparent again as the ammonia becomes the precursor of $\mathrm{ZnO}$, as is shown in the equation below [88]:

$$
\begin{gathered}
\mathrm{NH}_{3}+\mathrm{H}_{2} \mathrm{O} \rightarrow \mathrm{NH}^{4+}+\mathrm{OH}^{-} \\
\mathrm{Zn}^{2+}+4 \mathrm{NH}_{3} \rightarrow \mathrm{Zn}\left(\mathrm{NH}_{3}\right)_{4}^{2+} \\
\mathrm{Zn}\left(\mathrm{NH}_{3}\right)_{4}^{2+}+2 \mathrm{OH}^{-} \rightarrow \mathrm{ZnO}+4 \mathrm{NH}_{3}+\mathrm{H}_{2} \mathrm{O}
\end{gathered}
$$

A more in-depth analysis of the growth was halted, because the growth did not work as well as the VLS method. The VLS method gave more uniform wires and also wires with better length to diameter ratios. Figure 5.6 (a) and (b) shows the difference in growth of the CVD method and the VLS method. The VLS method yielded longer nanowires, over $500 \mathrm{~nm}$ long, where wires grown by the CVD method were only about $200 \mathrm{~nm}$ long. The VLS method also yielded denser nanowires.

Table 5.2: VLS vs CVD

\begin{tabular}{|c|c|c|}
\hline & VLS & CVD \\
\hline Catalyst & Gold & Zinc Acetate \\
\hline Growth Temperature & $920^{\circ} \mathrm{C}$ & $95^{\circ} \mathrm{C}$ \\
\hline Growth Time & $30 \mathrm{~min}$ & 24 hours \\
\hline Density & Very dense & Less dense \\
\hline Length & $400 \mathrm{~nm}+$ & $300 \mathrm{~nm}$ max \\
\hline
\end{tabular}

Table 5.2 compares VLS vs CVD. Both methods have the disadvantage of requiring a catalyst. VLS has an added disadvantage, namely the high growth temperature compared to that of the CVD method. However, the 

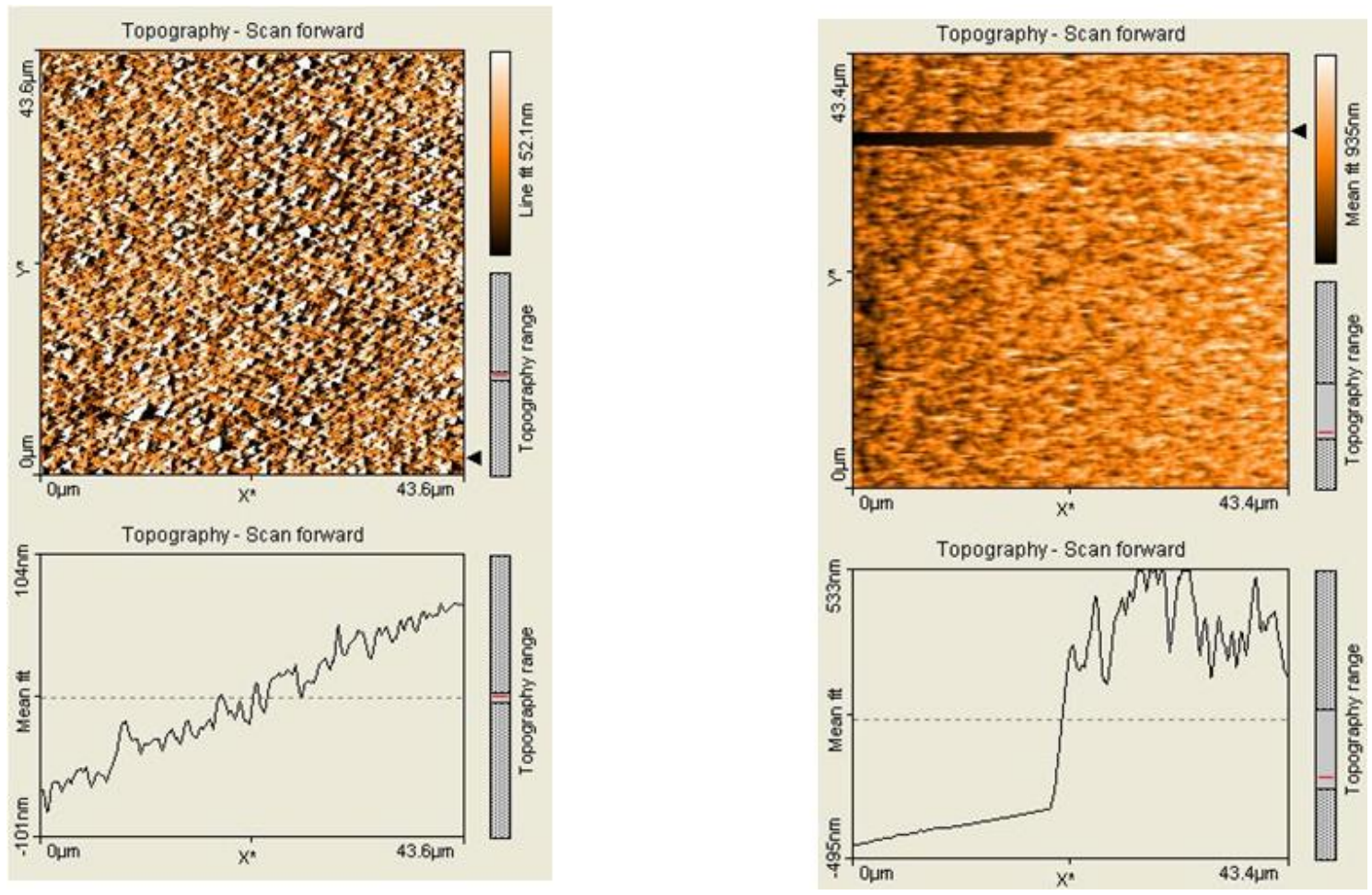

Figure 5.6: (a) CVD vs (b) VLS growth. Images obtained via AFM

growth time for VLS is much shorter than that of CVD. VLS also yielded longer nanowires than the CVD method. Results obtained with the AFM and light microscope are shown in Appendix A. It was easier to see the influence of certain parameters on the growth of the nanowires in this case, than it had been for the VLS method. The figures clearly show how the growth changed in some manner, whether it was the density of the nanowire growth or the length. This is much clearer than for the VLS method.

\subsubsection{Template Assisted Synthesis}

Template assisted synthesis is the easiest of all the methods used to grow nanostructures. A template is used to produce the desired structure. In the case of nanowires a disc, a few micrometers thick, with a diameter of a couple of millimeters [12], and through which many holes have been made, is used. Figure 5.7 shows a zoomed in image of one such membrane [81]. The product, from Whatman, comes in a thickness of $60 \mu \mathrm{m}$ and with three di- 
ameters: $13 \mathrm{~mm}, 21 \mathrm{~mm}$ and $43 \mathrm{~mm}$ [12]. This disc is placed in a solution to produce the desired nanowires. This solution and disc can then be subjected to different methods to produce the nanowires [79]. There are many different growth methods, from electroplating to a CVD method, which may be used to produce the desired structure with the template. This method is by far the easiest for the growth of nanowires, but the initial cost of the template makes this method impractical, because there are cheaper methods that are not much more difficult. The obvious advantage of this method is the ease with which growth can be accomplished, just by using the template and then electroplating, or some other supported method. However, the cost of the template is the main disadvantage. The template assisted synthesis method was not considered for this project.

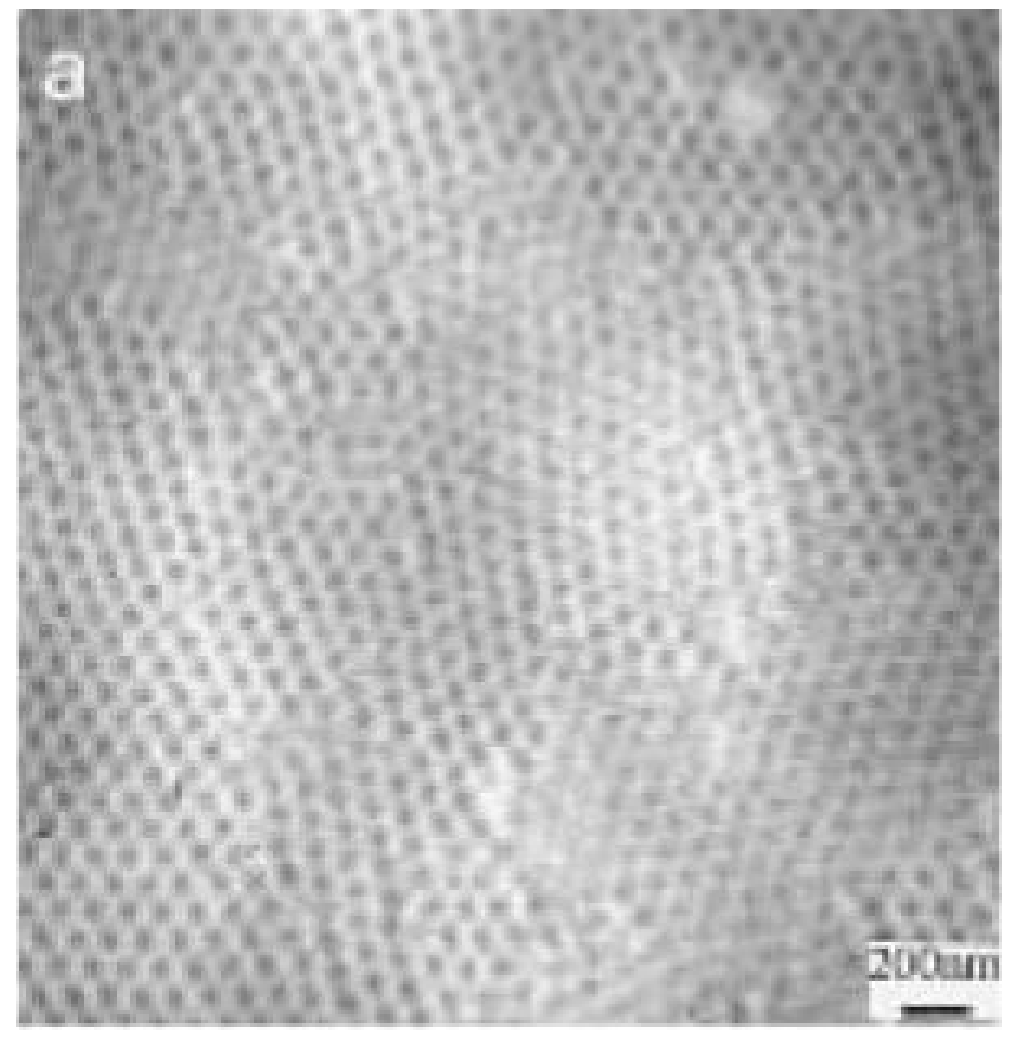

Figure 5.7: Picture of a template used for nanowire growth [12]. 


\subsubsection{Electrospinning}

Electrospinning is a technique for making fibre mats from a precursor solution. In recent years the technique was modified in order to make long fibres or nanowires by this method [13]. Electrospinning uses a needle to inject a specified solution into an electric field. The electric field overcomes the solution's surface tension and thus creates the long fibres. The method is visualized in Figure 5.8.

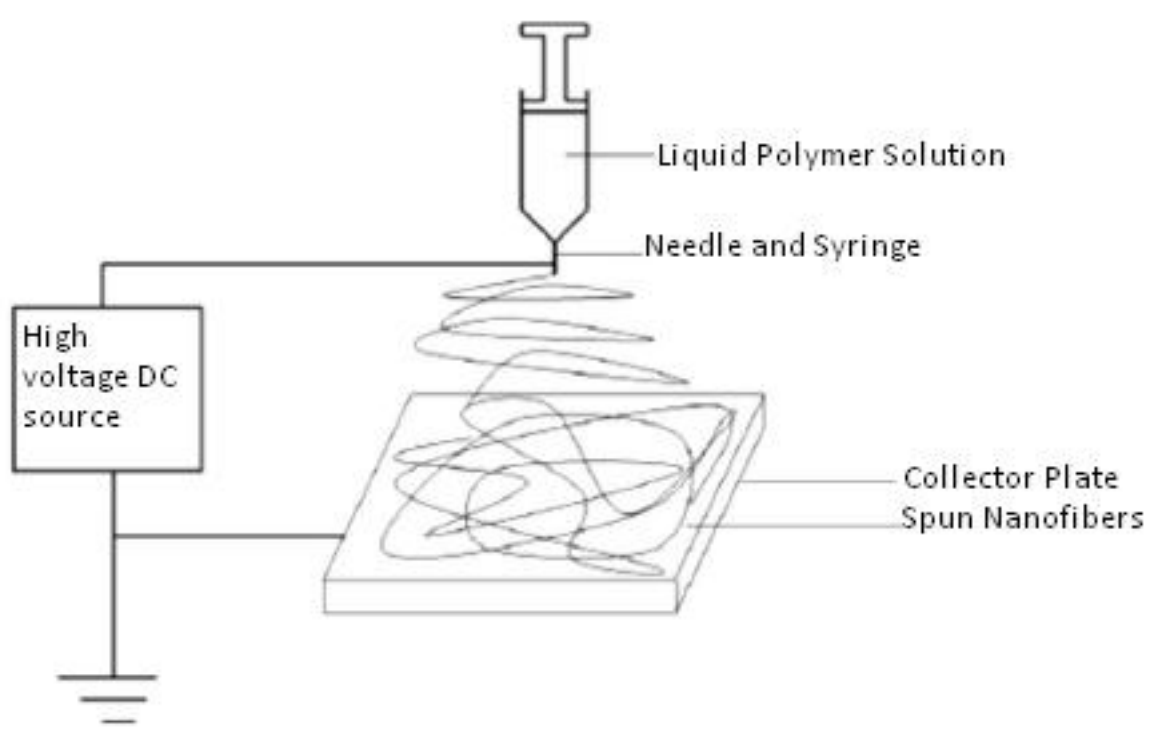

Figure 5.8: Electrospinning setup [13].

The needle at the top contains a certain liquid polymer solution. This solution contains the specific materials to make the fibres. The needle should be as thin as possible to ensure that the stream of liquid that is present in the electric field is as fine as possible. The high voltage DC source can be used in a number of different setups. The DC source can range from five to 20 kilovolts, positive and/or negative. Figure 5.8 shows just one possible setup, where the positive voltage is connected to the needle and the collector plate is at ground potential. This is, however, not the only setup - the needle can be at ground potential while the collector plate is at either a positive or a negative voltage. The final option is to have both the needle and the collector plate at a set voltage. The collector plate is to collect the spun fibres. 
The needle injects the polymer solution into the electric field. The electric field penetrates the solution and causes certain parameters of the solution to change. The stream of solution becomes increasingly thin as it travels through the electric field and up to the point where it is collected on the collector plate [89].

The spun nanofibres are collected on the collector plate in no specific orientation. The fibres fall onto the plate and on top of each other, which makes it difficult to use the fibres for any specific applications. Advances in the collection of the fibres are making it increasingly possible to collect the fibres in an ordered manner. Using a cylinder that spins at a certain speed to collect the fibres, it is possible to collect the fibres in one long continuous filament [13].

In order to make the nanofibres from a desired material, the polymer solution must contain that material in some form. To make $\mathrm{ZnO}$ nanofibres, therefore, the polymer solution contained $\mathrm{ZnO}$. To make the solution more suitable for electrospinning, poly (vinylidene fluoride) (PVDF) was added to the solution. PVDF is a polymer that also exhibits the piezoelectric effect and is used often in electrospinning [89]. The piezoelectric effect in PVDF, however, is very weak and adding $\mathrm{ZnO}$ increases the piezoelectricity of the fibres. On the other hand, $\mathrm{ZnO}$ on its own cannot be electrospun, but adding PVDF to the solution makes it possible. Work done by Ogut et al. was used as a starting point to spin nanofibres containing $\mathrm{ZnO}$ [89].

The PVDF solution was made in collaboration with the Department of Polymer Sciences at Stellenbosch University. The thickness of the polymer solution plays an important role in the electrospinning process. If the dissolved material is too thick, it will simply travel through the electric field and fall onto the collector plate without any change in properties, because the electric field would be unable to penetrate the solution due it its thickness. If the dissolved material is too thin, it will travel through the electric field, which would penetrate it, causing it to become even thinner. It will then break up into smaller pieces and fall onto the collector plate. The fibres will then be small droplets, not continuous fibres as desired. In order to make the solution, knowledge of polymers is a necessity, which is why the polymer solution was prepared at Polymer Sciences.

Making $\mathrm{ZnO}$ nanowires by the electrospinning method was briefly investigated. This method, however, was not feasible due to a number of reasons: 
1. The author's knowledge of polymers is very limited and the precise preparation and properties of the solution are critical to understand the exact mechanisms of the method.

2. Polymer Sciences has only one lecturer who has the required knowledge of the polymer solution used for this project and he was very busy during the time of this project. Help was thus limited.

3. The nanofibres are not aligned or in any specific order, but are randomly distributed on the collector plate.

4. The spun fibres are too brittle and break too easily when subjected to an external force. [89].

No feasible results were obtained with the electrospinning method. Due to the problems listed above, the method was not considered any further. Extensive research in electrospinning would make it possible to use this method to make $\mathrm{ZnO}$ nanofibres for use in a pressure sensor, but with the VLS method working and producing good results, gaining the necessary knowledge to perform electrospinning seemed redundant.

\subsubsection{Electrodeposition}

The last method that was tried for growing $\mathrm{ZnO}$ nanowires was electrodeposition. Electrodeposition is a method that uses electrical current to reduce cations of the desired material from a solution. These cations are deposited as a thin layer on a conductive substrate [90]. Electrodeposition is a very attractive method to synthesise $\mathrm{ZnO}$ nanaowires, because the growth happens at a low temperature.

To make $\mathrm{ZnO}$ nanowires by electrodeposition, the solution used was $\left.\mathrm{Zn}(\mathrm{NH})_{3}\right)_{4}\left(\mathrm{NO}_{3}\right)_{2}$ [82]. The solution had a concentration of $0.1 \mathrm{M}$. The solution is also known as the electrolyte. The electrolyte was made by mixing zinc nitrate hexahydrate $\left(\mathrm{Zn}\left(\mathrm{NO}_{3}\right)_{2} 6 \mathrm{H}_{2} \mathrm{O}\right)$ and ammonium $\left(\mathrm{NH}_{3}\right)$. The solution must be clear and have a $\mathrm{pH}$ value of 10 [82]. A silicon substrate was then placed in the solution. The substrate was connected to a copper block, which was connected to a voltage source. The copper acted as the cathode. A zinc rod that was placed in the solution acted as the anode. The positive side of the voltage source was connected to the anode (zinc rod) and the negative side to the 
cathode (copper block). The solution was heated to about $70^{\circ} \mathrm{C}$ and the voltage source was turned on. A constant current of $0.8 \mathrm{~mA}$ flowed during the growth of the nanowires [82].

The chemical reactions taking place are described below:

$$
\begin{gathered}
\mathrm{Zn} \rightarrow \mathrm{Zn}^{2+}+2 e^{-} \\
\mathrm{Zn}\left(\mathrm{NH}_{3}\right)_{4}\left(\mathrm{NO}_{3}\right)_{2} \rightarrow \mathrm{Zn}\left(\mathrm{NH}_{3}\right)_{4}^{2+}+2 \mathrm{NO}_{3}^{-} \\
\mathrm{Zn}\left(\mathrm{NH}_{3}\right)_{4}^{2+} \rightarrow \mathrm{Zn}^{2+}+4 \mathrm{NH}_{3} \\
\mathrm{NH}_{3}+3 \mathrm{H}_{2} \mathrm{O}+2 e^{-} \rightarrow \mathrm{NH}_{4}^{+} 3 \mathrm{OH}^{-}+\mathrm{H}_{2} \\
\mathrm{Zn}^{2+}+2 \mathrm{OH}^{-} \rightarrow \mathrm{Zn}(\mathrm{OH})_{2} \rightarrow \mathrm{ZnO}+\mathrm{H}_{2} \mathrm{O}
\end{gathered}
$$

When the anode and cathode are placed inside the electrolyte, the anode will dissolve and form $\mathrm{Zn}^{2+}$ ions. These ions will move to the cathode under the applied electric field [82]. This gives a brief description of the mechanism for electrodeposition of $\mathrm{ZnO}$ nanowires.

There are a couple of important parameters to consider in the growth of the $\mathrm{ZnO}$ nanowires. The length of time over which the reaction takes place determines the length of the nanowires. When the reaction is left for 4 hours the nanowires will be about $5 \mu \mathrm{m}$. The length increased further with an increase in time. The next important parameter is the concentration of the solution. The concentration determined the diameter of the nanowires. A concentration of $0.1 \mathrm{M}$ yields nanowires with a diameter of about $100 \mathrm{~nm}$ and the diameter increases as the concentration increases. The last important parameter was the current that flowed through the system. If the current is too low, a thin film will develop, and not nanowires. If the current is too high, other structures will form, and also no nanowires. A current of $0.8 \mathrm{~mA}$ yielded the best $\mathrm{ZnO}$ nanowires in previous work [82].

The experiments done for this thesis were performed with the following parameters: Reaction time of 3 hours; concentration of $0.1 \mathrm{M}$ and a current of $0.8 \mathrm{~mA}$. According to Zhang et al [82] this should have yielded perfectly aligned $\mathrm{ZnO}$ nanowires of about $100 \mathrm{~nm}$ diameter and $5 \mu \mathrm{m}$ in length. This was, however, not the case. Figure 5.9 shows a SEM picture of a sample grown with the above parameters. The figure shows perfectly grown $\mathrm{ZnO}$ nanowires, with different diameters. The length was too large to measure 
with the AFM which has a maximum scan height of $4 \mu \mathrm{m}$.

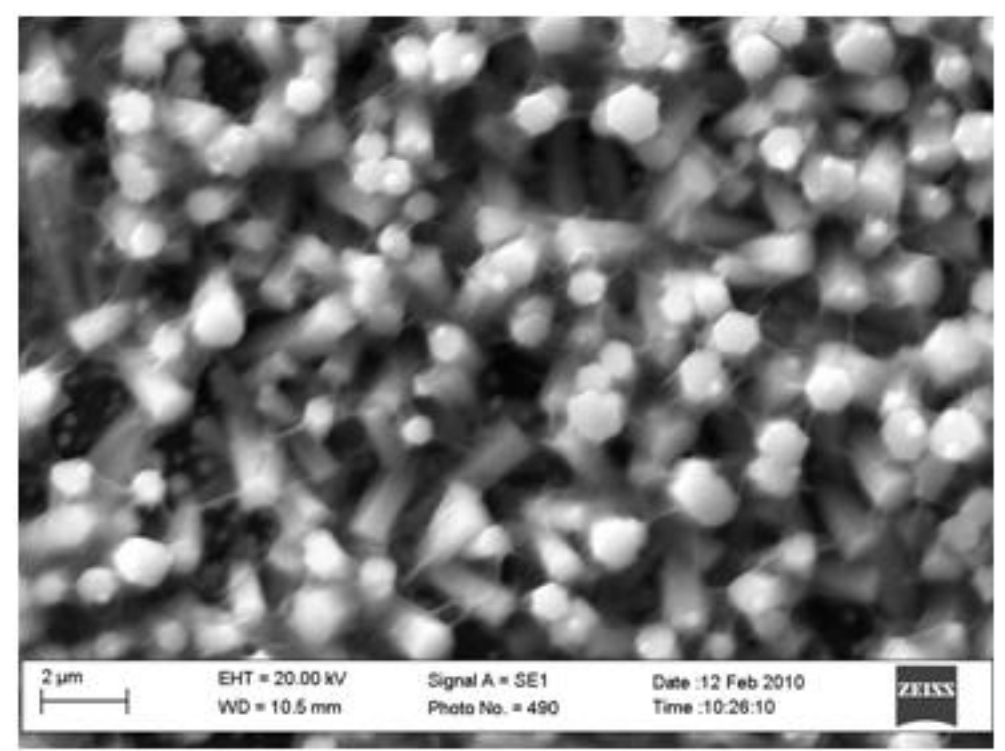

Figure 5.9: SEM picture of $\mathrm{ZnO}$ nanowire growth with electrodeposition

The growth from electrodeposition looked very good, except that the growth shown in Figure 5.9 happened only at specific regions on the sample. Most of the sample had a thick layer on it. The layer had a white colour, which was not $\mathrm{ZnO}$ due to the ammonium, which formed during the nanowire growth. Different solution concentrations, time and current were used, but to no avail. The samples never looked as good as the samples obtained via the VLS method.

Electrodeposition holds a lot of promise. If the entire sample could yield growth as shown in Figure 5.9, it would be the best and most economical method to grow the $\mathrm{ZnO}$ nanowires. More knowledge of chemistry is also necessary to determine why the growth occured only in specific areas.

A number of methods were investigated for the growth of $\mathrm{ZnO}$ nanowires. The methods listed above are just a few of the methods that are being used in practice today. Methods that are not listed above were not considered, because they use equipment or materials that are not easily obtainable in South Africa. Each of the methods listed above was investigated in attempts to produce nanowires that could be used to make a pressure sensor. Each method 
had clear advantages above other methods, but in the end the VLS method was used to grow the nanowires, because of its simplicity.

\subsection{Uses of nanostructures}

More and more everyday products are incorporating nanotechnology to make them more efficient. Nanostructures have not yet really infiltrated the consumer market. Most commercial products that incorporate nanotechnology use nanoparticles, or other nano related products. Nanostructures are, however, becoming more popular as manufacturing or growing these structures becomes more easily controlled and economically feasible.

There is a limited number of products that use nanostructures to perform a certain task. Nanostructures are used mostly for measuring devices, such as pressure sensors or gas sensors. All these products are, however, still in the experimental phase and no commercial products are available yet. 


\section{Chapter 6}

\section{Laboratory Equipment}

\subsection{Scanning Tunneling Microscope}

The STM was invented in 1981 by two IBM workers, Gerd Binnig and Heinrich Rohrer. The invention of the STM also resulted in them winning the Nobel Prize for Physics in 1986 [14]. The STM is used to look at surfaces at the atomic level. The STM has a small and very sharp tip or needle that scans very close to the sample, but does not touch it. The needle is so close to the sample that electrons tunnel between the surface and the needle, producing an electrical signal [91]. As the needle gets closer to the sample, only a few atoms away, the tunneling current increases [92]. The STM always tries to keep the needle at a distance of one atom's diameter away from the sample, giving very good resolution of the sample it is scanning [14, 91].

The STM used in this project is the easyScan 2 STM from Nanosurf [93] as seen in Figure 6.1. This STM has a platinum-iridium tip that can move in three dimensions using piezo-crystal translators driven with sub-nanometer precision [14]. The tip approaches the sample to within one nanometer, which is close enough for the tunneling current to exist. The strength of the tunneling current depends on the distance between the tip and sample, which makes it possible to measure the sample surface very precisely [14]. The Zpiezo translator can now be used in a feedback loop that keeps the tunneling current constant by keeping the distance between the tip and sample constant. By moving the other two translators, the sample can now be scanned. In the Z-direction the STM can move $200 \mathrm{~nm}$ with a $3 \mathrm{pm}$ resolution and in the $X$ and $Y$-directions the STM can move with a 15 pm resolution. The main 
disadvantage of the STM, however, is that the sample that is being scanned must be conductive [14]. The main advantage is the high resolution one can obtain with the STM.

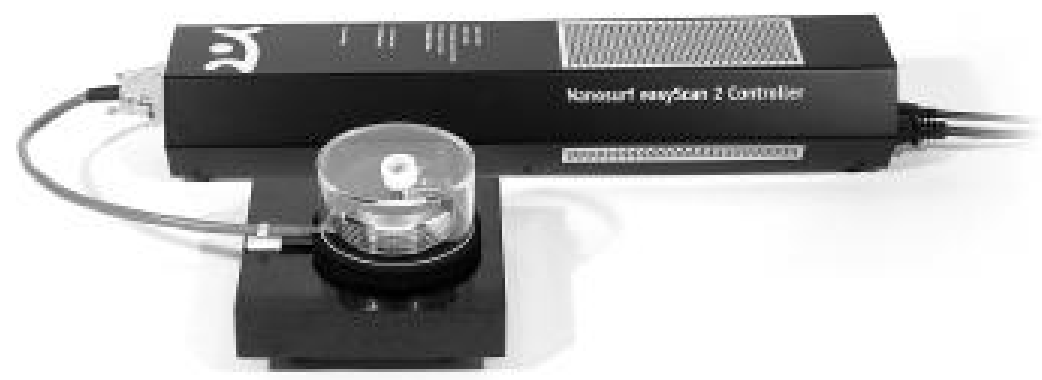

Figure 6.1: Picture of Nanosurf easyScan 2.0 STM [14].

\subsection{Atomic Force Microscope}

Because the STM is limited to conductive samples, another microscope was necessary in order to see samples that are non-conductive. The AFM is an extension of the STM and works on roughly the same principles [15]. The AFM was invented by Gerd Binning, Calvin Quate and Christoph Gerber in 1986 [94]. Once again, there are no optical lenses or anything similar with which to see the object, but rather a sharp tip that is very close to the sample surface, only a few nanometers, and atomic-range forces are acting between the tip and surface. In the AFM there is a tip attached to the free end of a cantilever that measures these atomic forces [15].

The AFM works on the same principle as when you run your finger over a sample and feel the surface to establih what it looks like. The tip on the cantilever is very small, only $2 \mathrm{~nm}$ radius, so it can scan over the surface with very high resolution [95]. The atomic forces acting between the tip and surface are not measured directly, but are calculated by measuring the deflection of the cantilever as shown schematically in Figure 6.2. The AFM can work under different modes. In static mode the tip is in contact with the surface and the deflection of the tip is being measured as the tip scans over the surface. Dynamic mode uses vibrations at a certain frequency, and measures 
changes in this vibration during a scan over the surface. The tip does not touch the sample, but is kept at a fixed distance [15].

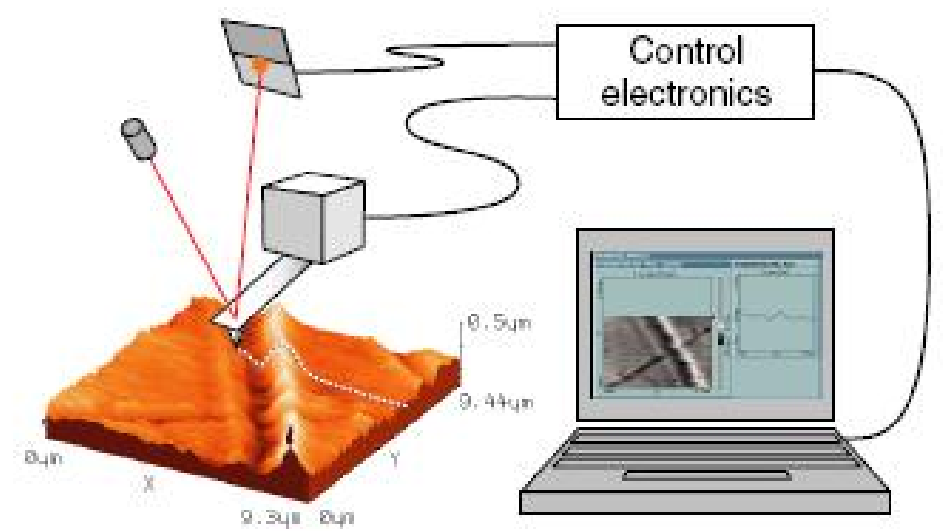

Figure 6.2: Picture of the internal operation of the AFM [15].

The AFM used for this project is the easyScan 2 from Nanosurf [93] and is shown in Figure 6.3. The AFM cantilever can move in the $X$ and $Y$ directions to scan a sample and also move in the $\mathrm{Z}$ direction to create depth in the image. The AFM can move $50 \mu \mathrm{m}$ in the $X$ and $Y$ directions with a resolution of $1.1 \mathrm{~nm}$, but this depends on the type of cantilever being used. The $\mathrm{Z}$ direction has a resolution of $0.21 \mathrm{~nm}$ and a range of $14 \mu \mathrm{m}$ with the same type of cantilever [15]. The main advantage of the AFM over the STM is the fact that any type of surface can be scanned, not just conductive samples. The main disadvantage is that the resolution of the AFM is not as good as that of the STM.

\subsection{Scanning Electron Microscope}

The Scanning Electron Microscope (SEM) used for this project was at the Stellenbosch University Geology Department. Appointments had to be made in advance to use the SEM. Each visit to the SEM also costs a few hundred rand, depending on the time spent and type of analysis required. The SEM scans a picture by use of a high-energy beam of electrons in a specific pattern. The electrons interact with the atoms of the sample being scanned to produce a picture. The electrons scatter on the sample and bounce back, and are then 


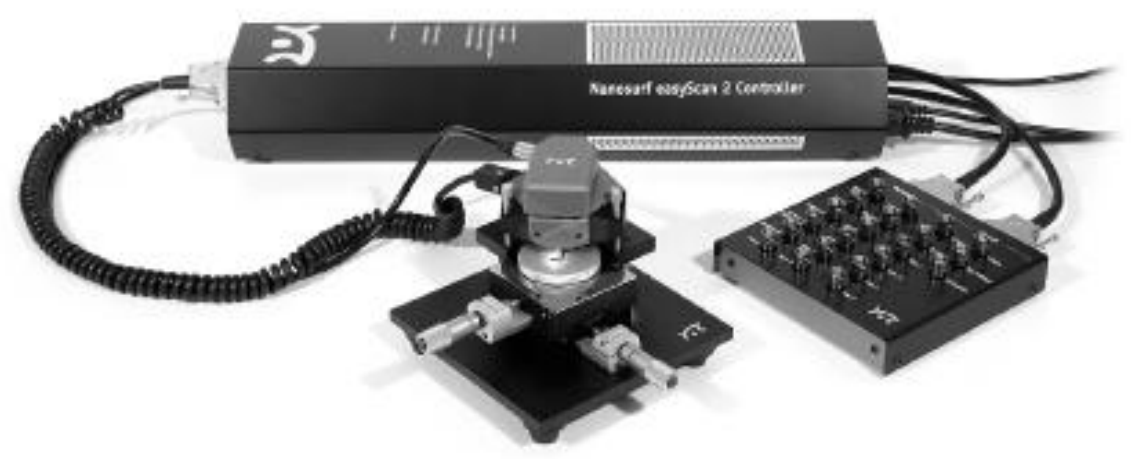

Figure 6.3: Picture of Nanosurf easyScan 2.0 AFM [15].

measured, along with some of the electron's properties, and a picture drawn from the data.

The SEM used was the Zeiss EVO MA15 SEM. The samples were mounted on a stub with double sided carbon tape to make the samples electrically conductive. Most pictures were taken at $7 \mathrm{kV}$ and $1.5 \mathrm{nA}$, with a working distance of $13 \mathrm{~mm}$.

\subsection{Microscopes}

Two optical light microscopes were also used. One microscope from Windaus [96] has a zoom of 1.5 times maximum. A black and white camera, Moticam $16 \mathrm{~mm}$, is connected to the microscope and also connected to a computer to enable the pictures to be viewed. With Motic Images Plus 2.0 software measurements can be made. The other microscope is from Olympus [97] and has interchangeable lenses with zooms from 3 times to 30 times. The microscope has a colour camera, Moticam 1000, which is also connected to a computer to make all sorts of software measurements, as well as to save pictures. Figure 6.4 shows the two microscopes that was used and Figure 6.5 shows the software.

\subsection{Furnace}

The furnace used for the growth of the $\mathrm{ZnO}$ NWs was made in-house at Stellenbosch University. The furnace itself is a pipe furnace made of asbestos as 


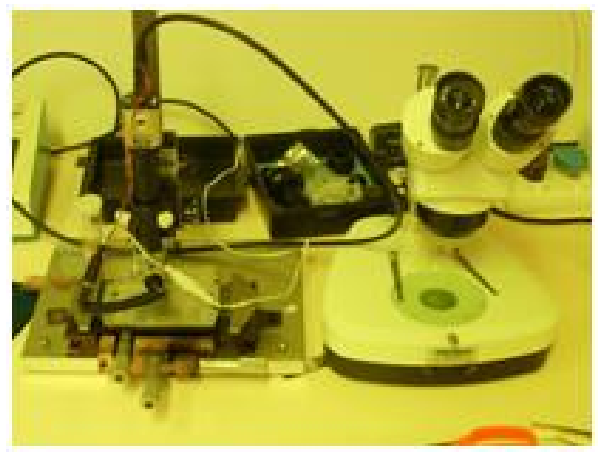

Figure 6.4: Picture of the two microscope systems. The one on the left can magnify up to 30 times and the one on the right to 1.5 times.

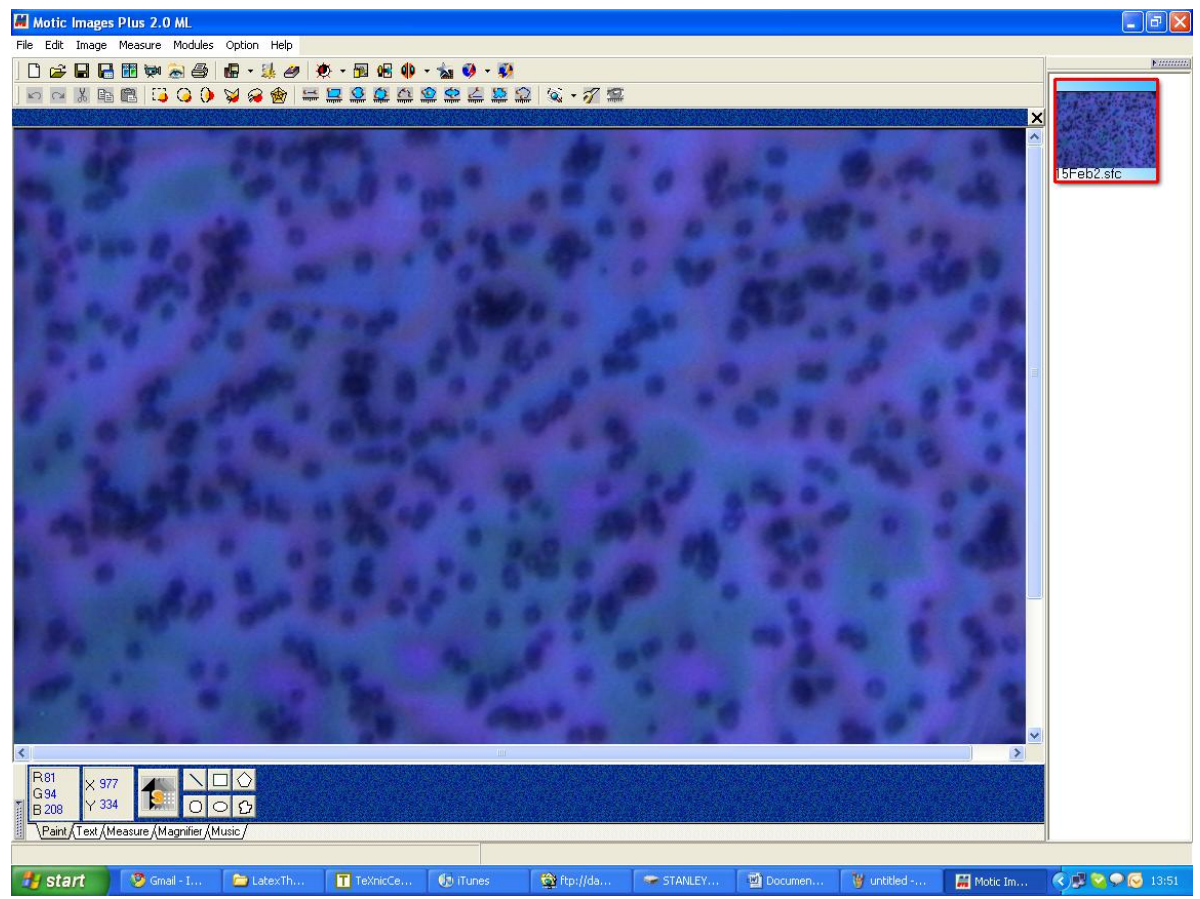

Figure 6.5: Screenshot of the software, Motic Images, used with the microscopes. 
an insulator of heat. The heating element is cylindrical and fits inside the asbestos covering. The furnace has a hole through the centre through which a tube can fit. The materials for growth are then loaded into the tube and the tube is placed in the furnace. The two ends of the tube are fitted with stoppers. Pipes for carrying gas are connected to the tube and a temperature probe is placed inside the furnace. A controller box can be set up to control the temperature. Specific temperatures can be given, the ramp time and the duration at a particular temperature can also be specified. The controller box will then start to heat the furnace and, by means of the temperature probe inside the furnace, compare the true temperature and the desired temperature and act accordingly. Gas flow meters are connected between the gas bottles and the furnace. The flow meters have valves to accurately control the rate of flow of the gas through the system. Figure 6.6 shows the complete furnace setup and Figure 6.7 shows the software that controls the furnace.

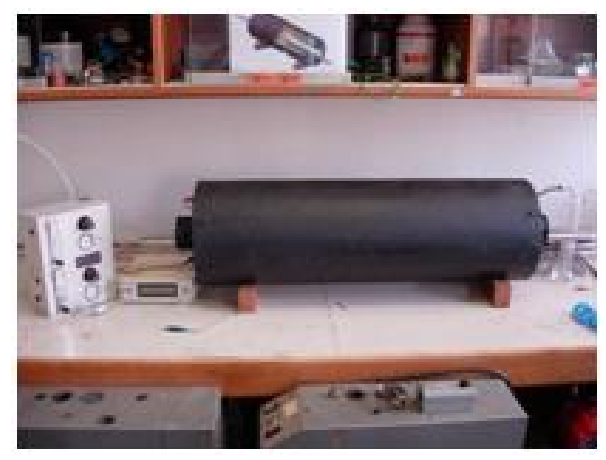

Figure 6.6: Picture of the tube furnace used for VLS growth. On the left are the gas bottles (out of sight) with the valve box just in picture. The furnace is the black object in the centre. Between the furnace and the valve box is the controller box.

\subsection{Sputter Unit}

A high voltage sputter unit, Edwards Sputter Coater S150B, was used to deposit thin layers of gold onto substrates. To start with, the sputter unit must be put under vacuum to remove impurities in the air. When the vacuum has reached a specified point $\left(4 \times 10^{-2} \mathrm{mbar}\right)$, a valve is opened to reduce the vacuum. When the vacuum is at a desired point (4 mbar), a high voltage is applied to a circular disc. The gold target, which is also a circular disc, is 


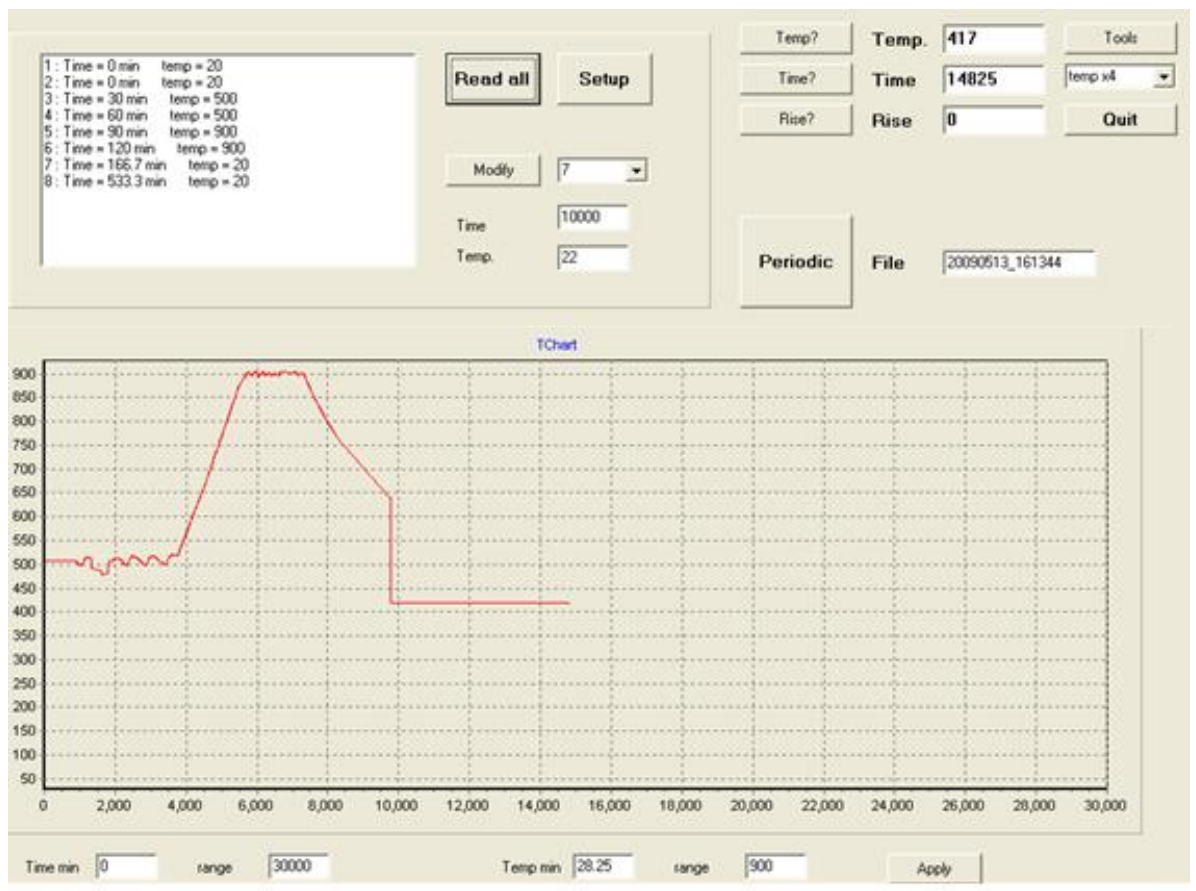

Figure 6.7: Software used with the furnace. The graph at the bottom shows temperature vs time. At the top are all the settings used to change the temperature and time.

placed a few millimeters away from the high voltage disc. Because the system is under vacuum, but not too high, the high voltage energizes particles in the air. These particles hit the gold target and loosen the gold atoms. The voltage breaks the gold atoms apart and the gold atoms fall down to a ground level, and onto the sample. The high voltage does not work under high vacuum conditions, because there will not be enough molecules in the air to hit the gold target and loosen the gold atoms. There is a timer on the sputter unit to set the desired time for the sputtering process to continue. Figure 6.8 shows the Edwards Sputter Coater S150B.

\subsection{Spin Table}

A spin table from Headway Research Incorporated [98] was used to spin photoresist on samples and is shown if Figure 6.9. The sample is placed in the centre of a circular disc, with a centre hole, connected to the laboratory's vacuum pump system. This holds the sample securely by suction, preventing 


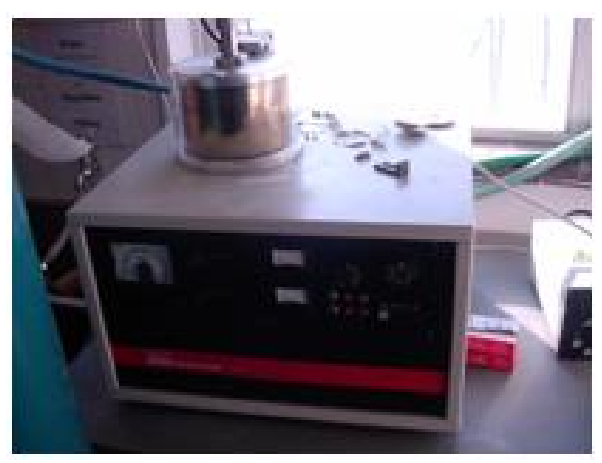

Figure 6.8: Edwards Sputter Coater S150B

it from spinning off during the process. When the sample is in place, fluid can be placed on the sample and the spinning process can begin. Because this is a rather old spin table, only a manual dial is present with which to choose the spinning speed, which is is then displayed on a screen. In other words, the speed cannot be preset. The dial can be turned to the desired speed and then left in position for the duration of the the spin process. There are two pedals on the floor, one to start the spinning and one to stop. The spin table has a range of 0 - $15000 \mathrm{rpm}$ (revolutions per minute).

\subsection{Ultrasonic Mixer}

A supersonic cleaner, MRC Supersonic Cleaner DG-1, was used to clean samples. The samples were placed in a glass holder with acetone or de-ionized water, which was then placed in the supersonic cleaner, filled with water. Supersonic waves are sent through the liquid in the supersonic cleaner, which in turn vibrates the glass holder and the liquid inside it, cleaning the sample. Figure 6.10 shows the ultrasonic bath.

\subsection{Hotplate}

A hotplate was used to soft bake samples after photoresist had been spun onto them. The hotplate has a temperature controller to set the temperature at the desired value and it is then kept at that temperature until it is switched off. The hotplate can reach temperatures of up to $250^{\circ} \mathrm{C}$. Figure 6.11 shows the hotplate that was used. 


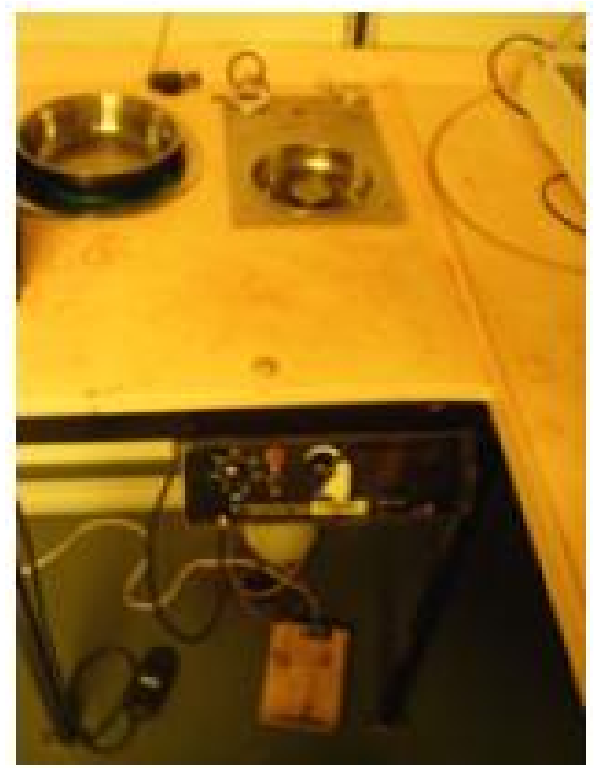

Figure 6.9: Picture of the spin table. At the back, the silver area is the spinning area. The black box connected to the table is the control box, used to set the speed. On the floor, the black pedals are to start and stop the spinner.

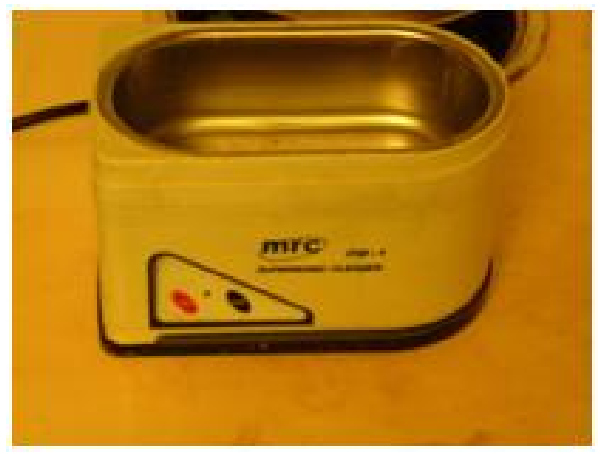

Figure 6.10: Picture of the ultrasonic mixer. 


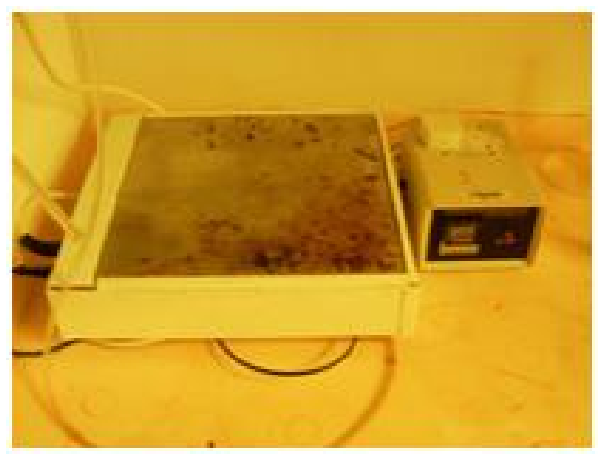

Figure 6.11: Picture of the hotplate. On the left is the hotplate itself and on the right the controller box.

\subsection{Sample Cutter}

A sample cutter from Karl Süss [99] was used to cut large silicon discs into $10 \times 10 \mathrm{~mm}$ substrates. The cutter has an even surface with holes in it, which are connected to the vacuum system. This vacuum suction system holds the disc that is up for cutting firmly, making sure that it will not move during cutting. The cutter has a sharp diamond-like tip that is very sharp, in order to cut trenches into the sample. The instrument is shown in Figure 6.12. The cutting lines can be viewed with the overhead microscope. The surface with the holes in can then be rotated by 90 degrees to cut out squares. The microscope has two lines, one vertical and one horizontal, in order to align the sample perfectly. The surface is moved via a roller, which has a distance scale attached, to accurately move the sample by a desired amount.

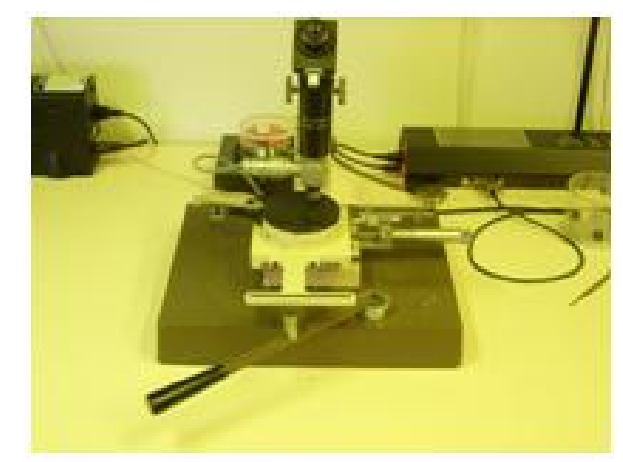

Figure 6.12: Picture of the sample cutter. 


\subsection{Scale}

For measuring powders and liquids for mixing, a scale with $0.1 \mathrm{~g}$ accuracy was used. The scale is from Kern Tee. The maximum weight it can measure is $150 \mathrm{~g}$ [100]. Figure 6.13 shows the scale that was used.

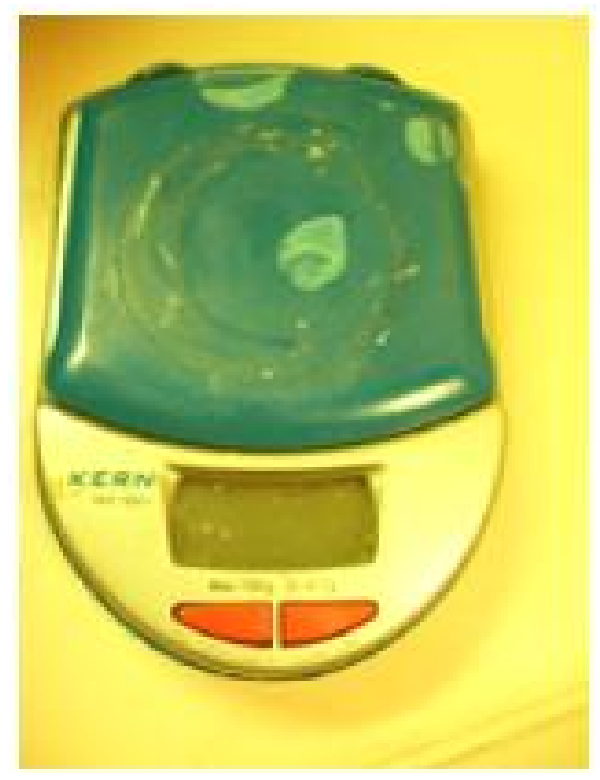

Figure 6.13: Picture of the scale.

\subsection{Lithography}

Lithography was done by an ultra violet (UV) lithography and alignment setup by Karl Süss [99]. The system is old, so no information is available online. Specific pattern samples are placed and fastened, by vacuum, to the system. The sample on which the pattern must be imprinted is then aligned precisely with the overhead pattern, using the onboard microscope. Once the sample and pattern are aligned, as desired, the lithography process can begin. Lithography is a very simple and effective method of imprinting patterns onto samples. A UV light is shone through a pattern and the light falls onto the sample that has some sort of photoresist on it. The light is reflected at a certain point and, depending on the pattern and the sort of photoresist, 
positive or negative, the pattern will emerge on the sample. The time duration for the lithography can be preset. The UV light will move forward, open its shutter and the light will expose the pattern, and after the preset time, the shutter will close and the UV light will move back into position. The sample can be removed and then developed to see if the pattern came out as desired. One drawback to lithography is that it has a limit on how small the pattern can be. It depends greatly on the wavelength of the UV light source. A pattern with $1 \mu \mathrm{m}$ lines was successfully manufactured using the lithography unit at Stellenbosch University. Figure 6.14 shows the lithography setup.

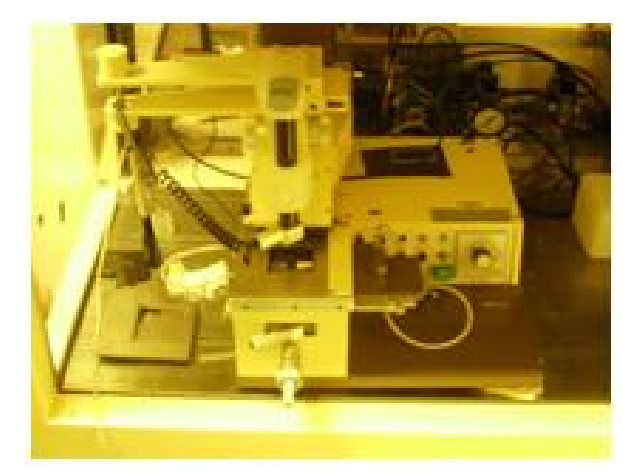

Figure 6.14: Picture of the lithography system.

\subsection{Mixer}

A magnetic stirrer, the CAT magnetic Mixer, was used to stir materials. The stirrer has two dials; one to change the temperature and one to choose the rotation speed. A small magnetic bar is placed in the material that is being stirred. Underneath the surface are two metal blocks that turn around, moving the magnetic bar, and thus stirring the material. Figure 6.15 shows the magnetic stirrer.

\subsection{High-Voltage source}

The high voltage source used for Electrospinning was built by Mr. Ulrich Büttner and Mr. Pieter de Kock. The voltage source can reach $25 \mathrm{kV}$ per channel and there are two channels, so the maximum voltage is $50 \mathrm{kV}$. There 


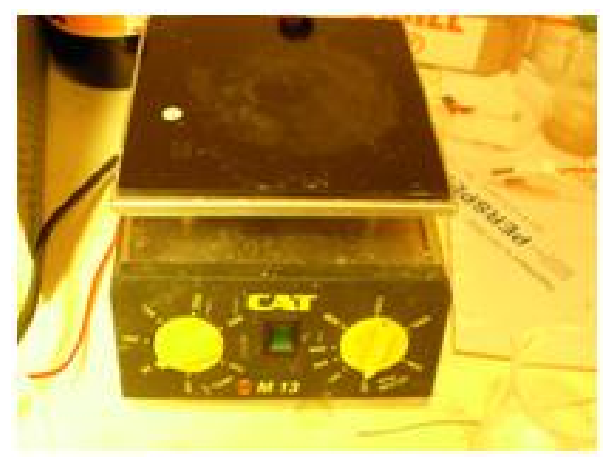

Figure 6.15: Picture of the mixer. A flask with chemicals in it is placed on top and a small white magnet is placed inside. The dials are then turned to spin the magnet and heat up the flask.

are also two ground probes, one to ground the substrate and the other to discharge the positive probe after electrospinning.

\subsection{Thermal Evaporator}

The thermal evaporator is the most used system in the laboratory. It is a very simple but effective method of depositing a layer of a specific material onto a substrate. As the name implies, it works by evaporating the source material and this vapour is then collected onto the sample. The system has its own vacuum system, in order to get a good vacuum before evaporation. This is done to remove any impurities that are contained within the system. The source material is then loaded into a boat made of a material, such as tungsten, that will not break under high heat. The substrate is then placed directly above the source material, upside down. In other words, the desired surface is facing the source material. A sensor is placed right next to the substrate to measures the thickness of the material, and also the rate of deposition. The vacuum pump is then turned on. It takes twenty minutes for a good vacuum to form. Once the vacuum is at the desired value, a program that is connected to the sensor to show the rate of deposition, and also the thickness of the material on the substrate, is started. Because all materials are not the same, certain values, which are dependant on the source material, must be loaded into the program beforehand. There are three parameters that can be changed: density, z-factor and tooling factor. The density of a material is 
easy to find and self explanatory. The z-factor is frequency dependant and is defined as the ratio between sound pressure and particle velocity. The zfactor of each material is different and must be known in order to calibrate the system. The tooling-factor is the last parameter that can be altered. The tooling-factor takes into account the system and different setups. The source material and sensor are not always at the exact same position and the ratio of the respective amounts of deposit between these two surfaces is known as the tooling-factor [101]. The tooling factor is not often changed. If the true thickness and the thickness given by the program differs, the z-factor is changed until both values match. Once all the parameters are loaded and the vacuum is at the desired value, the evaporation can begin. By turning a knob the current that goes through the boat where the source material is loaded increases, which in turn increases the temperature of the boat. Once the temperature reaches a certain point, the material will start to evaporate. The sensor will pick this up and the thermal evaporation is under way. Once the desired thickness of the material deposited on the substrate is reached, the system is turned off and a valve is opened to let the pressure return to normal. Calibration is always necessary, because the parameters that were loaded into the software might not be correct and then the thickness will be incorrect. As mentioned above, the z-factor is usually changed when there is a difference in true thickness and the thickness shown by the software. Figure 6.16 shows the thermal evaporator setup.

\subsection{Sonic Mill}

The Sonic Mill is used to drill holes into silicon or glass substrates. Normal drilling equipment will crack or break the samples. The Sonic Mill uses a specialized bit that vibrates at a constant frequency, to drill the hole as desired. The bit can be changed to any size and any form. The sample is placed underneath the bit. The system is entirely automatic. After the sample is in place, the system is turned on. Black slurry constantly flows over the sample. The bit then slowly moves downward and starts to cut into the sample. When the bit has gone far enough, it will retract and turn off the machine. The sample now has a hole in it. The slurry is essential for the drilling process. The bit moves down and some of the slurry gets in between the bit and the sample. The slurry then vibrates due to the vibration of the bit. It is actually the slurry 


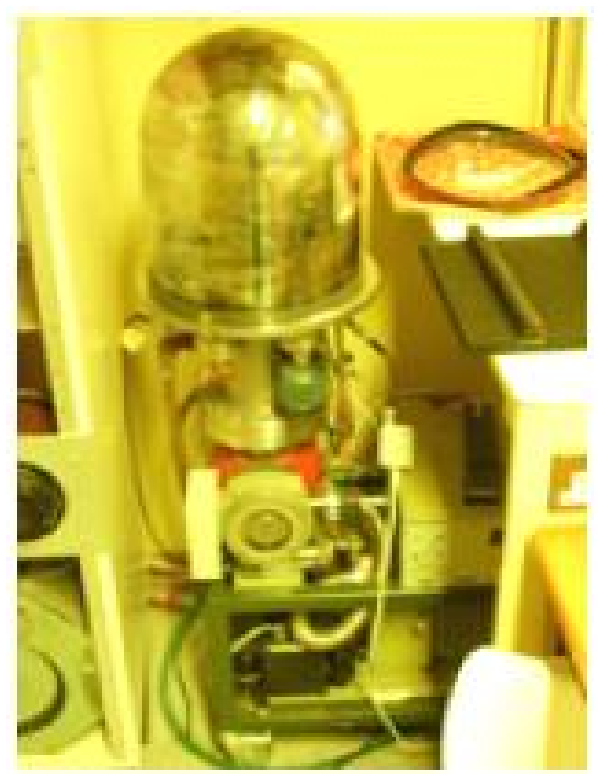

Figure 6.16: Picture of the thermal evaporator. The sample is loaded into the glass dome with the source material. The system is then put under vacuum to achieve the thermal evaporation.

that drills the hole, and not the bit itself. If the slurry were not present, the bit would do the drilling and the sample would break. Figure 6.17 shows the sonic mill. 


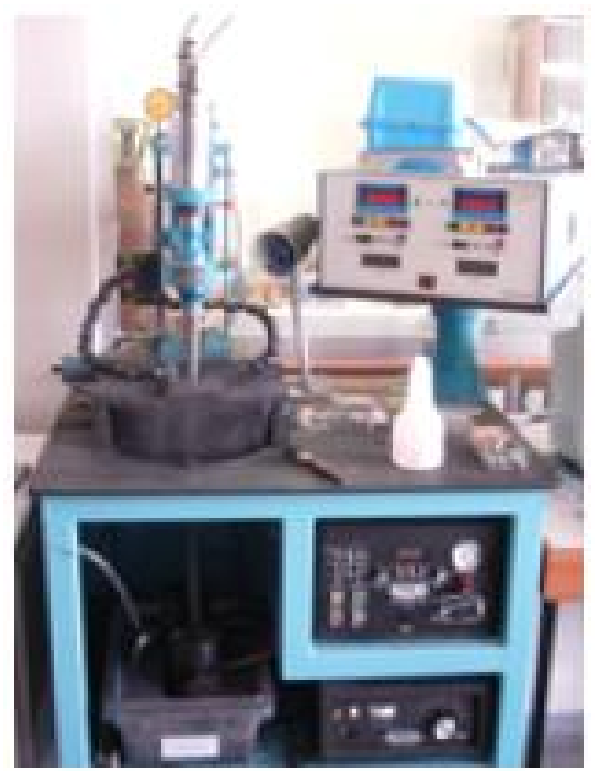

Figure 6.17: Picture of the Sonic Mill. To the left is the bit, at the top right is a motor used to position the bit. Bottom left is a bucket with the slurry. Bottom right are the two controller boxes for the system. 


\section{Chapter 7}

\section{Simulations}

\subsection{Introduction}

Simulation plays an important role in any experiment. Once the experiment has been defined and the desired outcome is known, the experiment can be simulated to ensure that the outcome is possible. A simulation also gives a better understanding of what will be necessary to achieve the desired result.

Simulations can transpire in many different ways. Formula 1 racing car design teams use wind tunnels to simulate racing conditions in order to optimise the aerodynamics of the car. Structural engineers that design tall skyscrapers make a small model of the building and then simulate different natural occurrences that the building will encounter to ensure that the building will not collapse.

The results obtained from the simulations can then be used to optimise the product. If the F1 team designed a car without a wind tunnel, during a race the car would not perform as well as it could have and at that stage nothing can be done. Simulations thus provide an easy and effective way to see how a product will behave under certain conditions. Simulations are, however, just an imitation of the real thing and the end product will probably differ from the simulation. Most of the time simulations do not account for all the imperfections and losses in a real system.

Comsol [102] is a company that provides software to simulate a broad range of physical phenomena [103]. This includes acoustics, heat transfer, structural mechanics, MEMS and many more [104]. For this project, Comsol Multiphysics was used to simulate $\mathrm{ZnO}$ nanowires and see how much 
potential the wires could generate in relation to the applied pressure.

\subsection{Comsol Simulations}

Comsol Multiphysics with the MEMS module was used to do the simulations of the $\mathrm{ZnO}$ nanowires. The MEMS module was used, because it adds unique properties to materials at the nanoscale. For this project the piezoelectric effect of $\mathrm{ZnO}$ nanowires was simulated.

The simulation is performed in a 3D space environment with 'Piezo Solid Structural' properties. For the purpose of simulation the nanowires were drawn as a uniform cylinder with a fixed length and radius, as shown in Figure 7.1, in which the length is $600 \mathrm{~nm}$ and the radius $30 \mathrm{~nm}$. At this stage a cylinder with the desired dimensions has been created, but without structural or any other properties.

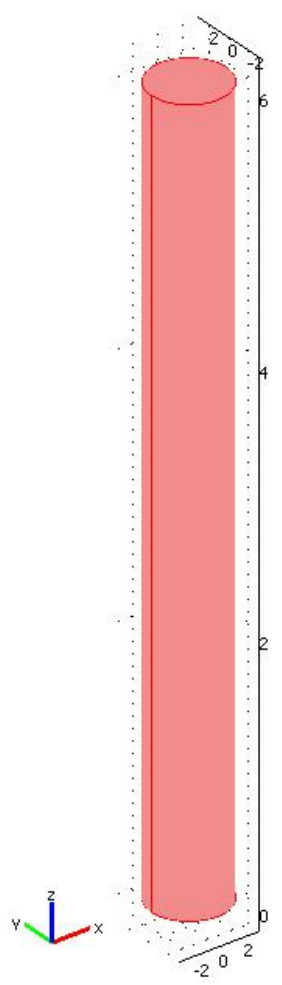

Figure 7.1: Nanowire drawn in Comsol Multiphysics with a length of $600 \mathrm{~nm}$ and a radius of $30 \mathrm{~nm}$ 
The cylinder must be made of $\mathrm{ZnO}$. To change the properties for simulations the 'Subdomain Settings' must be altered to the desired values. $\mathrm{ZnO}$ is a predefined material in the Comsol library, thus no further alteration is required. The $\mathrm{ZnO}$ properties that is defined in Comsol are the bulk material and not the nanoscale materials. There will be some difference in the properties of the bulk material and those of the nanoscale material, but these differences were ignored because the effect was very small [105]. No initial potential or charge was connected to the cylinder. The next set of settings that needed to be changed was the 'Boundary Settings'. Comsol splits the cylinder into six different boundaries: the top, the bottom and the cylinder, which is divided into four sectors. The bottom circle is fixed, in other words, it cannot move freely and it is also made to be on ground potential. The rest of the boundaries are left as is, free to move and with zero charge. The last step in the simulation is to define a pressure to bend the nanowire. In reality the force bending the wire will be small, but will also act on a small area. Pressure is defined as force per area, and the area in question here is very small, only a few nanometers in scale. The pressure chosen was thus extremely large, $40 \mathrm{MPa}$. This pressure was applied to the cylinder, and acted only in the x-direction.

Before the simulation can be started, the cylinder is broken down into small areas, called a mesh, in order to solve equations with a finite element method. All the equations are already part of Comsol and no mathematical input is required. The equations that are being solved are the same as the equations presented in Chapter 4 [62]. The mesh can be chosen to have more or fewer individual elements. If more elements are chosen, a convergence study must be performed to verufy the rsults. There is also a minimum number of mesh elements required in order to perform the simulation. Figure 7.2 shows the meshed nanowire, with the mesh consisting of 3023 elements.

Figure 7.3 shows the displacement of the top of the nanowire as it bends due to the pressure exerted on it. This bending of the nanowire causes a potential difference across the wire due to the piezoelectric effect, as described in Chapter 4. Figure 7.4 shows the potential difference across the wire with the $40 \mathrm{MPa}$ pressure applied to the top of the wire.

Figure 7.5 shows the displacement and Figure 7.6 shows the potential distribution of the nanowire with a mesh comprising of 11645 elements. As seen from the Figure 7.6, the simulation gives a different solution with a more 


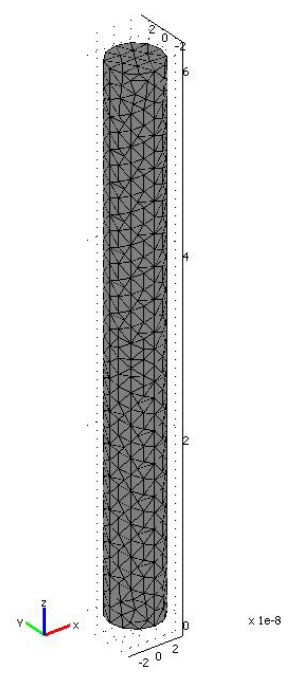

Figure 7.2: Meshed nanowire consisting of 3023 elements

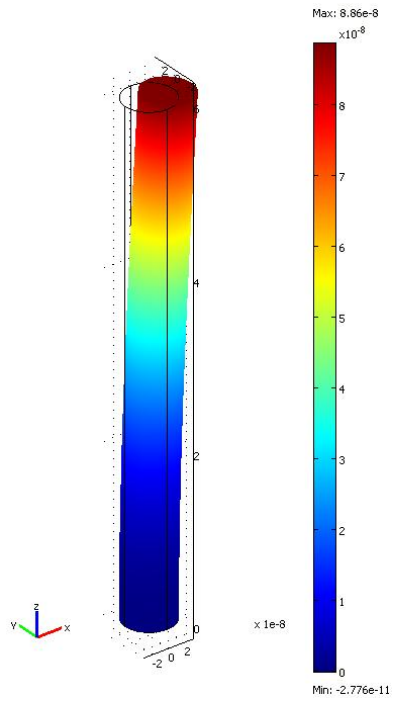

Figure 7.3: Displacement of nanowire when a pressure of $40 \mathrm{MPa}$ is applied to the top end of the nanowire 


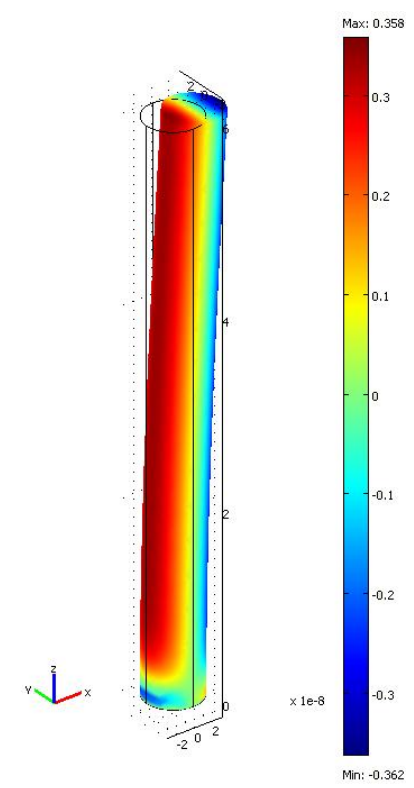

Figure 7.4: Electric potential generated in nanowire when a pressure of $40 \mathrm{MPa}$ is applied to the top end of the nanowire

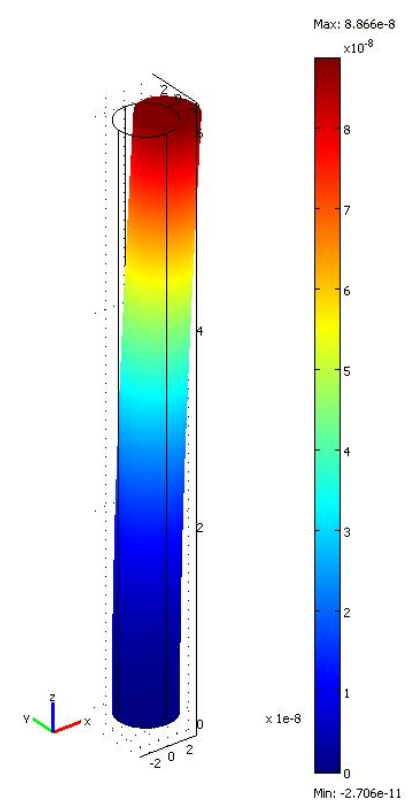

Figure 7.5: Displacement of nanowire with 11645 mesh elements 


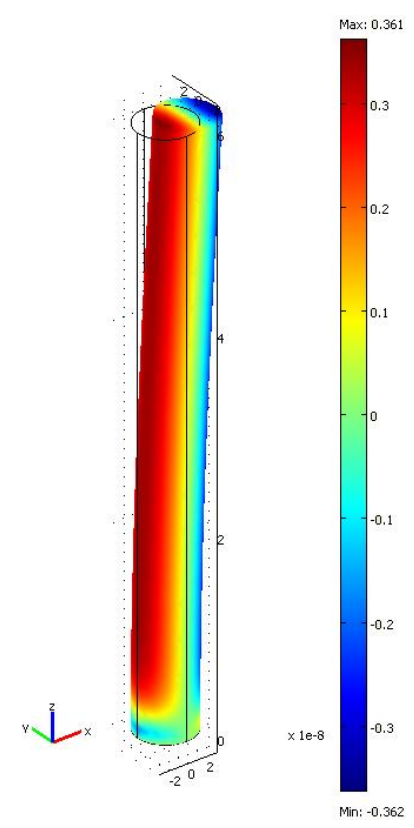

Figure 7.6: Electric potential generated in nanowire with 11645 mesh elements

defined mesh. Thus, as the number of mesh elements increases, the simulation results get better, but the simulation time expands. Table 7.1 shows that when the number of elements in the mesh increases, the electric potential theoretically becomes more accurate, but the time required to reach a solution increases.

Table 7.1: Relationship between number of mesh elements, electric potential and simulation time

\begin{tabular}{|c|c|c|}
\hline Mesh Elements & Maximum Electric Potential (V) & Simulation Time (sec) \\
\hline 3028 & 0.358 & 29.953 \\
\hline 11645 & 0.361 & 137.656 \\
\hline
\end{tabular}

In the next simulation, the number of mesh elements was more than 3.5 times higher than the minimum number required, and the simulation time increased by more than 4.6 times. The result obtained from the second run was only $2 \mathrm{mV}$ better, which is insignificant. The next number of mesh elements chosen was 36543, which was, again, more than 3 times higher than in the previous simulation and, with the same scaling, it will take more than ten minutes to perform the simulation. The computer used for the simula- 
tions did not have enough memory to perform the simulation for a mesh with more than 11645 elements.

The pressure exerted on the wire causes the wire to bend. The larger the pressure is, the larger the deformation will be and, thus, the larger the potential. Figures 7.7 and 7.8 show the simulation with a pressure of double the previous value, in other words $80 \mathrm{MPa}$. Table 7.2 shows the increase in displacement and potential as the pressure increases.

Table 7.2: Relationship between applied pressure, maximum displacement and maximum electric potential

\begin{tabular}{|c|c|c|}
\hline Pressure (MPa) & Maximum Electric Potential (V) & Maximum Displacement (nm) \\
\hline 20 & 0.179 & 44.3 \\
\hline 40 & 0.358 & 88.6 \\
\hline 60 & 0.537 & 132.9 \\
\hline 80 & 0.716 & 177.2 \\
\hline 100 & 0.894 & 221.5 \\
\hline 120 & 1.073 & 265.8 \\
\hline 160 & 1.432 & 354.4 \\
\hline 180 & 1.610 & 398.7 \\
\hline 200 & 1.788 & 443.0 \\
\hline 600 & 5.364 & 1329 \\
\hline
\end{tabular}

It is clear from Table 7.2 that when the pressure doubles, both the electric potential and the displacement double. There is a linear relationship between the pressure and potential and pressure and deformation. In practice the exact linear relationship will not be observed, because of certain imperfections which are not accounted for in the simulations. These imperfections include:

1. Capacitance of the wires.

2. Resistance of the wires.

3. The pressure will not always be working perpendicular to the wire.

4. The pressure will be exerted in more than one direction.

5. The wires can only bend a certain amount before breaking.

Unfortunately the Comsol MEMS package does not have the ability to simulate a pressure on one wire, which, as it bends, causes another wire to bend. This simulation can be done with non-piezoelectric materials as shown 


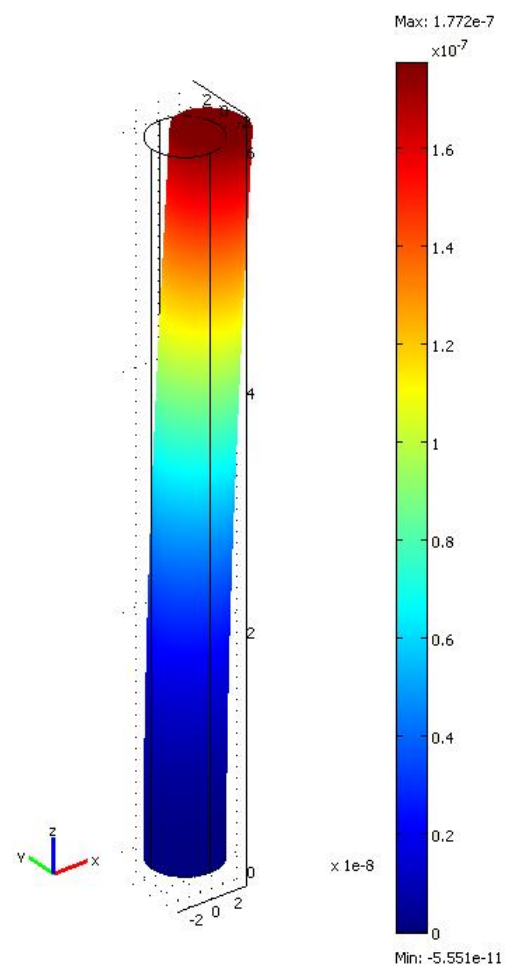

Figure 7.7: Displacement of nanowire when a pressure of $80 \mathrm{MPa}$ is applied to the top end of the nanowire

in Figure 7.9. The wire on the left is subjected to pressure of $40 \mathrm{MPa}$, which causes it to bend. Another wire, spaced just $1 \mathrm{~nm}$ from the first, gets bent, because the first wire, with pressure on it, bends and touches the next wire, causing it to bend, as shown in Figure 7.10. This will cause the piezoelectric effect to be exhibited in the second wire as well. This, however, cannot be simulated in Comsol, because the MEMS package does not support dependant structures.

The simulation uses the same mathematical equations as described in Chapter 4 . Solving the equations manually would be impractical, and thus the mentioned simulation environment was used instead. Solving the equations by means of the finite element method (FEM) will theoretically yield the same results as obtained by Comsol [62], because Comsol is also based on FEM.

The simulations were used to determine the range of the potential that is generated through the bending of the wire. Only one wire was simulated, 


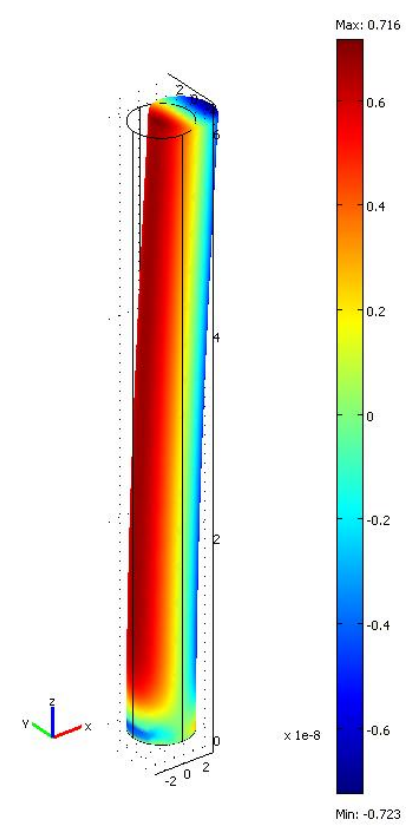

Figure 7.8: Electric potential generated in nanowire when a pressure of $80 \mathrm{MPa}$ is applied to the top end of the nanowire.

and this is sufficient, because the potential generated is dependent on the wire with the highest potential and not the sum of all the generated potentials, as has been described in Chapter 4. Simulations help to verify the theory of $\mathrm{ZnO}$ nanowires and the piezoelectric effect. The results obtained via the simulations were sufficient and proved that the theory will work in practice. 


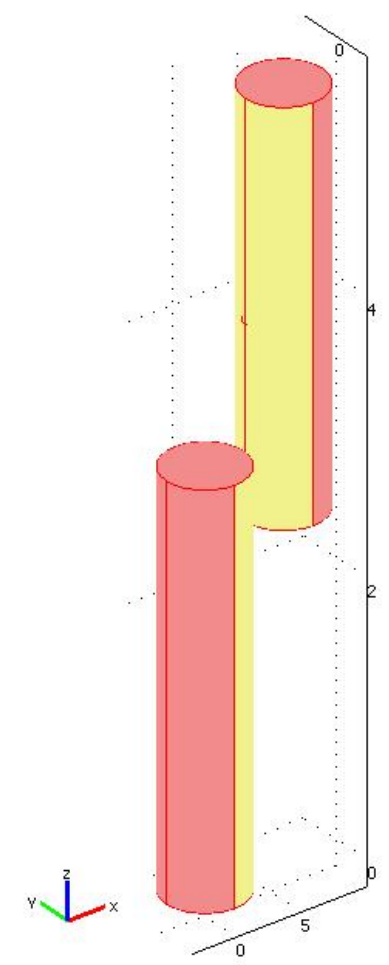

Figure 7.9: Two nanowires spaced $1 \mathrm{~nm}$ apart

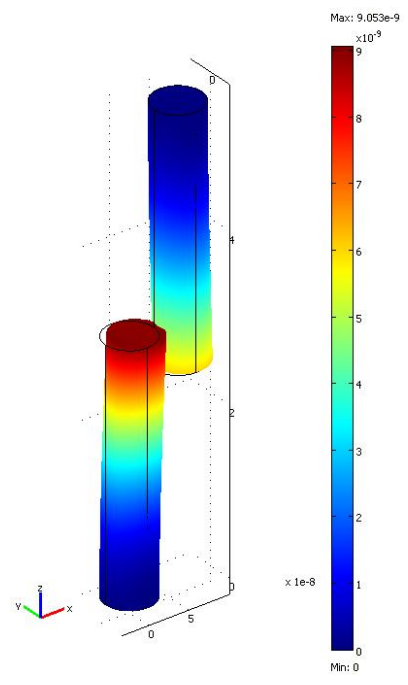

Figure 7.10: Two nanowires spaced $1 \mathrm{~nm}$ apart with pressure applied to the left nanowire, which causes the bending of both wires 


\section{Chapter 8}

\section{Design of Experiments}

\subsection{Theory}

Design of experiment is the design of all information gathering exercises where variation is present, whether under the full control of the experimenter or not [106]. The experimenter is interested in the effect of some process or the intervention on some objects [106]. Whenever experiments are performed, the repeatability of the experiment is critical. In most cases the results of the experiment will not be precisely the same, but will vary with some value. This is due to external factors not under the full control of the experimenter. Experiments can also have multiple inputs in order to get the desired output, and the interaction between these inputs also plays an important role. Design of experiments is the method used to calculate these interactions between inputs and also to take unwanted inputs into the system into account. Design of experiments is based on statistical data about the experiments, which is then used to calculate the most important inputs and also the interaction between inputs.

There are clear guidelines that must be followed during the experimentation phase and these are discussed in the paragraphs to follow [107]:

1. Recognition of, and statement of, the problem. The first step is to recognize the problem and make a clear statement of that problem. The problem must be characterized in full and all factors must be taken into account. After the problem is fully understood, it should be formulated and written down, so that the experimenter can always refer back to what exactly the problem is. 
2. Selection of the response variable. The response variable is the output variable of the experiment. The response variable must be measurable and also give useful information about the experiment. The response variable will be measured after each experiment, to see whether the results of the experiment have improved or not.

3. Choice of factors, levels, and range. This is one of the most crucial steps in the design of experiment method. All the factors that can influence the response variable within the experiment must be chosen. This includes variables that are not under the direct manipulation of the experimenter. The most important factors are the design factors and for these factors the maximum and minimum levels must also be chosen. It is often not possible to include all the factors that influence the response variable, but as many as possible should be found.

4. Choice of experimental design. Once all the factors are known, this step is rather easy. The experimenter should choose how many runs he/she wants to do, how many factors should be varied, and in which order. The experimenter must also know which of the factors are under his/her control and which are not. Not all of the factors will be altered in the experiment, so those factors which will remain constant throughout the experiment must be chosen.

5. Performing the experiment. During an experimental run the experiment must be monitored extensively to ensure that no unwanted factors are present, and also to ensure that all the experiments are performed under the same conditions. It is always good to first do a few trial runs to become familiar with the experimentation equipment. The experiments must also be performed in a random order, in order to minimize external influences.

6. Statistical analysis of the data. Statistical methods should be used to analyze the data from the experiment, so that an objective conclusion can be drawn.

7. Conclusions and recommendations. Using the statistical analysis of the data, a conclusion can be reached. The conclusion will be objective because of the statistical analysis and will give a clear indication of which factors of the experiment are of greater importance, and which are less 
important. This will also help in determining which parameters to vary in experiments that follow.

\subsection{Design of experiment applied to the optimization of $\mathrm{ZnO}$ nanowire growth}

Design of experiment can be very useful if the above steps are done correctly. It will simplify otherwise complex experiments and save the experimenter a great deal of time. For this thesis, design of experiments was used in the growth of $\mathrm{ZnO}$ nanowires, where there are a number of factors that can influence the growth of the nanowires. In the chapter that follows, the seven steps above are followed for the specific case of $\mathrm{ZnO}$ nanowire growth.

1. Recognition of, and statement of, the problem. The problem that was identified for this thesis was to grow $\mathrm{ZnO}$ nanowires with maximum length and good uniformity. The nanowires should also not be too thick. Growing long nanowires is not that difficult, but the problem is that the diameter also expands as the nanowire gets longer, and it will soon fall out of the nano range, and become microwires. It is easy to grow nanowires with diameters of only a few tens of nanometers, but then the length will be very short, only a few hundred nanometers. The aim was to get the maximum length, while still keeping the diameter in the nanoscale.

2. Selection of the response variable. As explained in the statement of the problem, the aim of the design of experiment in this thesis is to optimize the length of the $\mathrm{ZnO}$ nanowires. The response variable will be the length and will be measured using pictures generated by a scan by the AFM. Each sample will be scanned on a multiple of areas, in order to get an average height and diameter for the sample. The sample will be scanned on the top edge, in the middle and at the bottom edge, as shown in Figure 8.1 to Figure 8.3.

3. Choice of factors, levels, and range. As stated above, this is the most crucial step. In experiments done beforehand, some factors were seen to have a more profound effect on the growth of the nanowires than others. Table 8.1 lists the factors and the importance of their influence on the response variable, as seen from previous experiments. 
Table 8.1: Different factors that influence the growth of nanowires

\begin{tabular}{|c|c|}
\hline Factor & Effect on response variable \\
\hline System Temperature & High \\
\hline Growth Time & High \\
\hline Annealing Temperature & Medium \\
\hline Annealing Time & Medium \\
\hline Argon flow rate during growth & High \\
\hline Argon flow rate during annealing & Low \\
\hline Gold layer thickness & High \\
\hline Mass ZnO & Medium \\
\hline Mass Carbon & Medium \\
\hline Ratio of ZnO to Carbon & Medium \\
\hline
\end{tabular}

There are other factors that influence the gowth of $\mathrm{ZnO}$ nanowires as well, but the mentioned factors are some of the most important. As seen from Table 8.1, there are four factors that have a high impact on the response variable. These four factors were chosen as the variable factors, and their levels and range are shown in Table 8.2.

Table 8.2: Most important factors with their respective levels

\begin{tabular}{|c|c|c|}
\hline Factor & Low Level & High Level \\
\hline System Temperature & $700^{\circ} \mathrm{C}$ & $1000^{\circ} \mathrm{C}$ \\
\hline Growth Time & $10 \mathrm{~min}$ & $40 \mathrm{~min}$ \\
\hline Argon flow rate during growth & $60 \mathrm{sccm}$ & $120 \mathrm{sccm}$ \\
\hline Gold layer thickness & $10 \mathrm{~nm}$ & $20 \mathrm{~nm}$ \\
\hline
\end{tabular}

All the other parameters, shown in Table 8.3, were kept at constant values throughout each experimental run, as defined in Table 8.3.

These values were chosen through a combination of trial and error in previous experiments, and also from articles on the growth of zinc oxide nanowires [108]. External effects that influence the growth included factors that were not under the experimenter's direct control, and some that were under the experimenter's control, but did not receive any attention. These factors include pressure, humidity, temperature ramp time, cooling down time, cleaning of substrates and a few minor factors that are of less importance. 
Table 8.3: Different factors that were kept constant during each run

\begin{tabular}{|c|c|}
\hline Factor & Constant Level \\
\hline Annealing Temperature & $500^{\circ} \mathrm{C}$ \\
\hline Annealing Time & $30 \mathrm{~min}$ \\
\hline Argon flow rate during annealing & $60 \mathrm{sccm}$ \\
\hline Mass ZnO & $2 \mathrm{~g}$ \\
\hline Mass Carbon & $2 \mathrm{~g}$ \\
\hline Ratio of ZnO to Carbon & $1: 1$ \\
\hline
\end{tabular}

4. Choice of experimental design. In order to eliminate some of the external factors, listed above, that can affect the growth of the nanowires, two runs were done for each set of parameters. To further ensure that external factors were kept to a minimum, the order in which each parameter was altered was randomized. The parameter values for each of the experiments are shown in Table 8.4. Each measurement was also performed three times on a sample and an avarage was then taken. Figures 8.1 to Figure 8.3 show how the measurments were made using the AFM system.

Table 8.4: Experimental parameters

\begin{tabular}{|c|c|c|c|c|c|c|}
\hline Run & Temp $^{\circ} \mathbf{C}$ & Time(min) & Gold layer(nm & Ar flow(sccm) & Output(nm:nm) & Run Label \\
\hline 1 & $700^{\circ} \mathrm{C}$ & 10 & 20 & 120 & 115 & $\mathrm{~cd}$ \\
\hline 2 & $1000^{\circ} \mathrm{C}$ & 10 & 20 & 120 & 157 & $\mathrm{acd}$ \\
\hline 3 & $1000^{\circ} \mathrm{C}$ & 10 & 20 & 60 & 136.9 & $\mathrm{ac}$ \\
\hline 4 & $700^{\circ} \mathrm{C}$ & 40 & 10 & 120 & 112.2 & $\mathrm{bd}$ \\
\hline 5 & $1000^{\circ} \mathrm{C}$ & 10 & 10 & 60 & 103.9 & $\mathrm{a}$ \\
\hline 6 & $1000^{\circ} \mathrm{C}$ & 10 & 10 & 120 & 0 & $\mathrm{ad}$ \\
\hline 7 & $700^{\circ} \mathrm{C}$ & 10 & 20 & 60 & 174.0 & $\mathrm{C}$ \\
\hline 8 & $1000^{\circ} \mathrm{C}$ & 40 & 10 & 60 & 154.3 & $\mathrm{ab}$ \\
\hline 9 & $1000^{\circ} \mathrm{C}$ & 40 & 10 & 120 & 283.1 & $\mathrm{abd}$ \\
\hline 10 & $700^{\circ} \mathrm{C}$ & 10 & 10 & 120 & 0 & $\mathrm{~d}$ \\
\hline 11 & $700^{\circ} \mathrm{C}$ & 40 & 20 & 120 & 109.7 & $\mathrm{bcd}$ \\
\hline 12 & $700^{\circ} \mathrm{C}$ & 10 & 10 & 60 & 126.5 & $(1)$ \\
\hline 13 & $1000^{\circ} \mathrm{C}$ & 40 & 20 & 60 & 149.8 & $\mathrm{abc}$ \\
\hline 14 & $700^{\circ} \mathrm{C}$ & 40 & 10 & 60 & 224.4 & $\mathrm{~b}$ \\
\hline 15 & $1000^{\circ} \mathrm{C}$ & 40 & 20 & 120 & 199.7 & $\mathrm{abcd}$ \\
\hline 16 & $700^{\circ} \mathrm{C}$ & 40 & 20 & 60 & 146.8 & $\mathrm{bc}$ \\
\hline
\end{tabular}




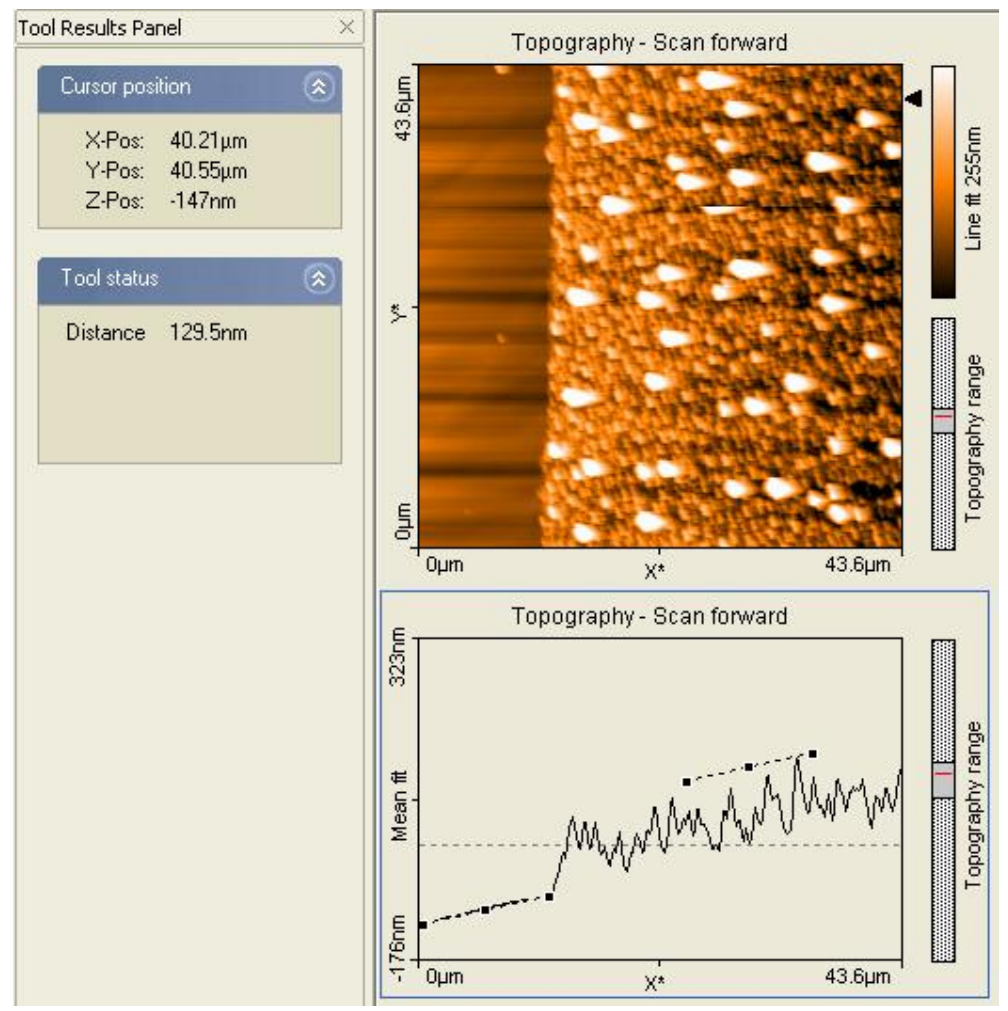

Figure 8.1: Measuring the height of nanowires at the top of a scan.

The reason for the 16 runs will be discussed when the statistical analysis of the data is discussed at point 6 . Each of these runs was performed twice. Each sample was then scanned at three places to get data as discussed above. All the data was collected to get a good average of nanowire growth with specific factors. Other factors that could influence the output were chosen and then kept at those values for the remainder of the experiment. These values include the distance from powder to substrate, which was $210 \mathrm{~mm}$, and the amount of powder, which was $2 \mathrm{~g} \mathrm{ZnO}$ and $2 \mathrm{~g}$ graphite. These were the only two critical values that were not varied [108].

5. Performing the experiment. The experiment was carried out twice for each of the unique sets of parameters, as listed in Table 8.4 above. The full detail regarding the experiment is discussed in Chapters 5 and 9. The mean value for the growth was $156.66 \mathrm{~nm}$, with a standard deviation of $2.79 \mathrm{~nm}$. 


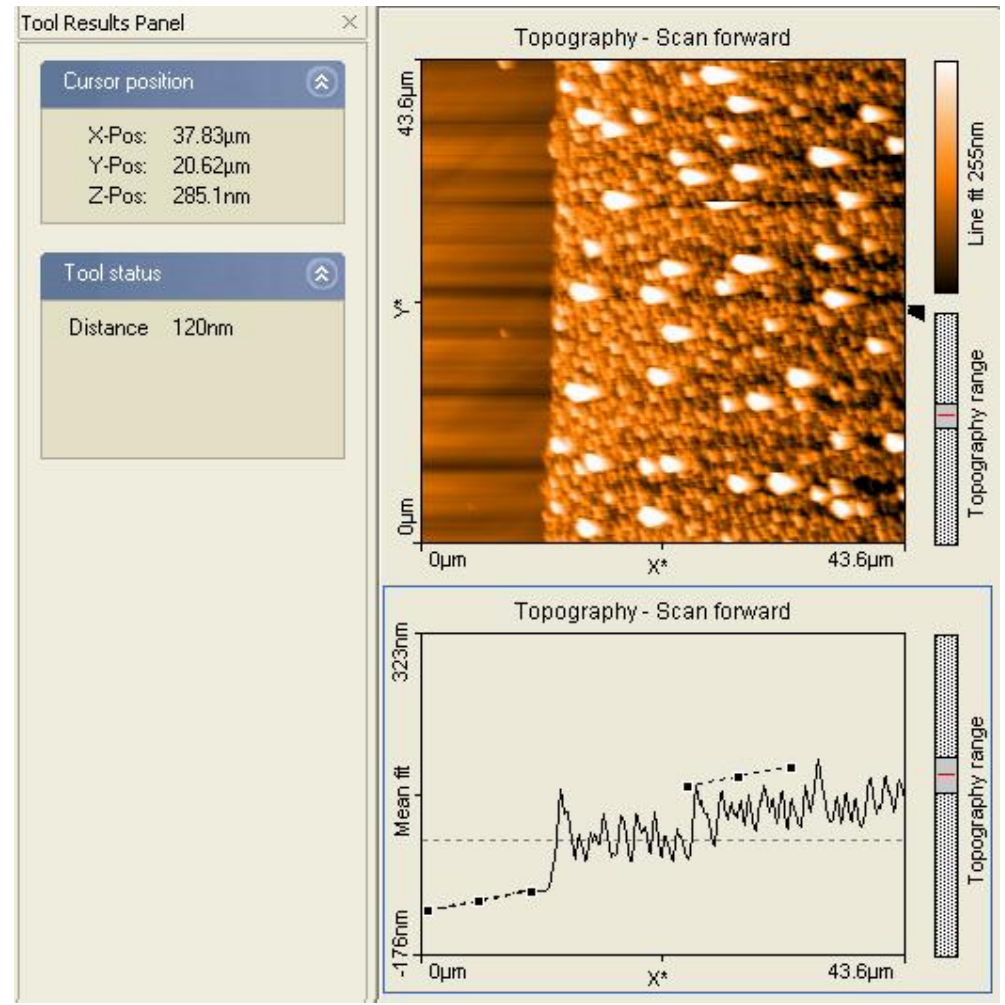

Figure 8.2: Measuring the height of nanowires in the middle of a scan.

6. Statistical analysis of the data. Choosing the type of statistical analysis to interpret the data is another crucial step. It does not help to choose a method that will not yield any relevant information about the data. For an experiment of this kind, with more than two input factors and a very precise output factor, factorial design works the best [109, 108]. A complete description and explanation of factorial design is beyond the scope of this thesis, but a brief introduction and an explanation of the most important formulas will be given.

In each complete replication of the experiment, all possible combinations of the levels of the factors are investigated and the effect of a factor is defined as the change in the response produced by the change in the level of the factor [109]. The $2^{k}$ factorial design is the most popular and easiest method to use whenever an experiment has $k$ factors that can influence the response. The input factors are denoted by uppercase letters (A, B, C, etc.) and the effects at different levels are denoted by 


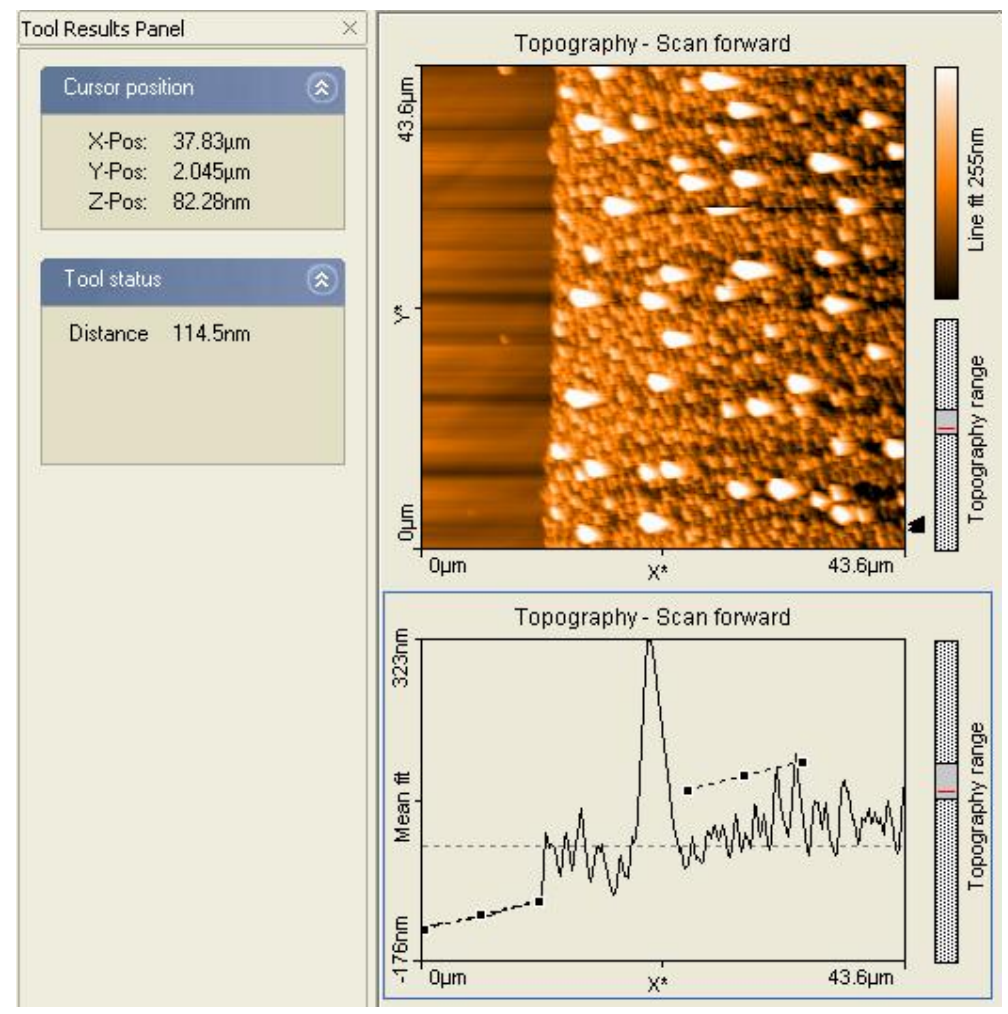

Figure 8.3: Measuring the height of nanowires at the bottom of a scan.

lowercase letters ( $a, b, c$, etc.). To get better results, more than one run is necessary for each set of factors; and $n$ denotes the number of runs per set. One of the most important equations for factorial design is the contrast of a factor. The contrast of a specific factor gives the total effect on the entire experiment due to a change in that specific factor. The contrast thus gives an indication of what factors have a large effect on the experiment. The contrast can be calculated for each separate factor, as well as for a combination of factors. For the experiment in this thesis there are four factors, and all the calculations shown below are thus for a $2^{4}$ factorial design. The contrast of factor $A$ is given by [109]

$$
A_{c}=[a+a b+a c+a d+a b c+a b d+a c d+a b c d-b-c-d-b c-b d-c d-b c d-(1)]
$$

where (1) denotes the set where no factor is changed and all factors are at their lowest level. The $a$ represents factor $A$ at its high level and the 
rest of the terms at the lowest level, and the same for the rest. Equation (8.2.1) is easier to understand, because the contrast of factor $A$ is due to the change in level of factor $A$, denoted by $a$. That is why all the sets with $a$ are added, and those without, are subtracted. Equation (8.2.1) can be rewritten as [109]

$$
A_{c}=(a-1)(b+1)(c+1)(d+1) .
$$

Equation (8.2.2) is just the factorized form of (8.2.1). As seen from (8.2.2), only the factor of which the contrast is being calculated has a minus one and the rest have plus one. The same principal applies in calculating all the other contrasts [109]. Equations for a few more are shown below.

$$
\begin{gathered}
B_{c}=(a+1)(b-1)(c+1)(d+1) \\
A B_{c}=(a-1)(b-1)(c+1)(d+1) \\
B C_{c}=(a+1)(b-1)(c-1)(d+1) \\
A B C_{c}=(a-1)(b-1)(c-1)(d+1) \\
A B C D_{c}=(a-1)(b-1)(c-1)(d-1)
\end{gathered}
$$

Once all the contrasts have been calculated, it becomes easy to calculate which factors and which interaction of factors play a deciding role in the outcome of the experiment. There are two criteria necessary to measure the influence of each factor and interaction of factors. The first is called the Estimated Effect (EE), and with the help of (8.2.2) [109] it can be expressed as

$$
\begin{aligned}
E E_{A} & =\frac{1}{8} n A_{c} \\
E E_{A B} & =\frac{1}{8} n A B_{c} \\
E E_{A B C} & =\frac{1}{8} n A B C_{c} \\
E E_{A B C D} & =\frac{1}{8} n A B C D_{c} .
\end{aligned}
$$

This gives an indication of how big an impact the factor has on the 
output. If the number is high, positive or negative, the influence is big and if the number is around zero, the influence is small. The second indicator is called The Sum of Squares (SS), and gives an indication of variance caused in the output due to an change in one of the factors. This gives a clearer indication of which factors are more important, and is calculated as [109]

$$
\begin{aligned}
S S_{A} & =\frac{A_{c}}{n 2^{n}} \\
S S_{A B} & =\frac{A B_{c}}{n 2^{n}} \\
S S_{A B C} & =\frac{A B C_{c}}{n 2^{n}} \\
S S_{A B C D} & =\frac{A B C D_{c}}{n 2^{n}} .
\end{aligned}
$$

Not all of the equations are shown, but from the above the rest can easily be derived.

Table 8.5 shows all the values for the experiment, as calculated, using the above equations. The percent contribution is calculated by taking the specific sum of squares for one factor and dividing it by the sum of the sum of squares and then multiplying that by one hundred.

Once all the above has been calculated, the most influential factors will emerge and can then be optimized. Choosing the optimal value for each factor is also not that difficult. Each factor has a high and a low value that is chosen at the beginning of the experimental phase, and the influence on the output due to the level of input can then be monitored, and from there, the level that yields the best output can be found. Although this does not give the optimal values, it does give an indication of the range in which the optimal values can be found.

7. Conclusions and recommendations. Table 8.5 shows all the important results from the 16 runs that were performed. The most important value is the Percent Contribution column. According to the software used, Stat-Ease Design Experiment 7 [110], all the values below 0.05 are significant in determining the height of the nanowires. The columns denoted by ' $\mathrm{NA}^{\prime}$, does not influence the growth of the nanowires in any 
Table 8.5: Statistical results

\begin{tabular}{|c|c|c|c|}
\hline Model Term & Effective Estimate & Sum of Squares & Percent Contribution \\
\hline A & 23.21 & 4310 & 0.0271 \\
\hline B & 24.66 & 6484 & 0.0221 \\
\hline C & -0.68 & 3.65 & 0.6183 \\
\hline D & -2.79 & 62.16 & 0.2167 \\
\hline AB & -0.43 & 1.98 & 0.7027 \\
\hline AC & -20.32 & 3304 & 0.0309 \\
\hline AD & 6.46 & 334.1 & 0.0965 \\
\hline BC & -12.42 & 1645 & 0.0438 \\
\hline BD & 40.51 & 17501 & 0.0134 \\
\hline CD & -0.48 & 1.81 & 0.7146 \\
\hline ABC & 11.44 & 1396 & 0.0475 \\
\hline ABD & NA & NA & NA \\
\hline ACD & NA & NA & NA \\
\hline BCD & -19.74 & 4158 & 0.0276 \\
\hline ABCD & NA & NA & NA \\
\hline
\end{tabular}

significant manner. In this case $\mathrm{A}, \mathrm{B}, \mathrm{AC}, \mathrm{BC}, \mathrm{BD}, \mathrm{ABC}$ and $\mathrm{BCD}$ are the significant terms. These terms coincide with temperature, time, temperature-Au thickness, time-Au thickness, time-Ar flow, temperaturetime-Au-thickness and time-Au thickness-Ar flow. The first two are obvious, the higher the temperature, the longer the nanowires, and the longer the time allowed for growth, the longer the nanowires (up to a certain point). The interactions between the parameters are where things start getting interesting. The combination between the temperature and the Au thickness is significant and looking at the EE a negative number is present. This means that it has a negative impact on the growth. When the gold layer is very thick and the temperature very low, the gold will not anneal enough and growth will be difficult. Time and the gold thickness also have a negative EE. The same reasoning applies. If the gold layer is too thick and the time too short, the gold will not anneal enough for significant growth. Time-Ar flow has a positive EE number. The right combination can thus result in longer nanowires. When the Ar-flow is very high and the time short, the nanowires will not easily grow. Temperature-time-Au-thickness gives a positive EE number. The same reasoning applies as for the separate cases, but it is interesting to see that the combination of the three gives a positive EE, 
where the combination of two gave a negative EE. The last significant combination is time-Au thickness-Ar flow, with a negative EE. Once again the same reasoning applies as stated above for the separate parameters.

The software also has the ability to give the optimal parameters. Looking at Figure 8.4, the two lines represent the two different Ar flow rates. The black line is $60 \mathrm{sccm}$ and the red line is $120 \mathrm{sccm}$. When the flow rate is $120 \mathrm{sccm}$, an increase in temperature also means an increase in nanowire length. However, when the flow rate is $60 \mathrm{sccm}$ the nanowire length decreases when temperature increases. Considering all the other graphs gives a set of optimal parameters that will give the longest nanowires possible with the parameters as chosen.

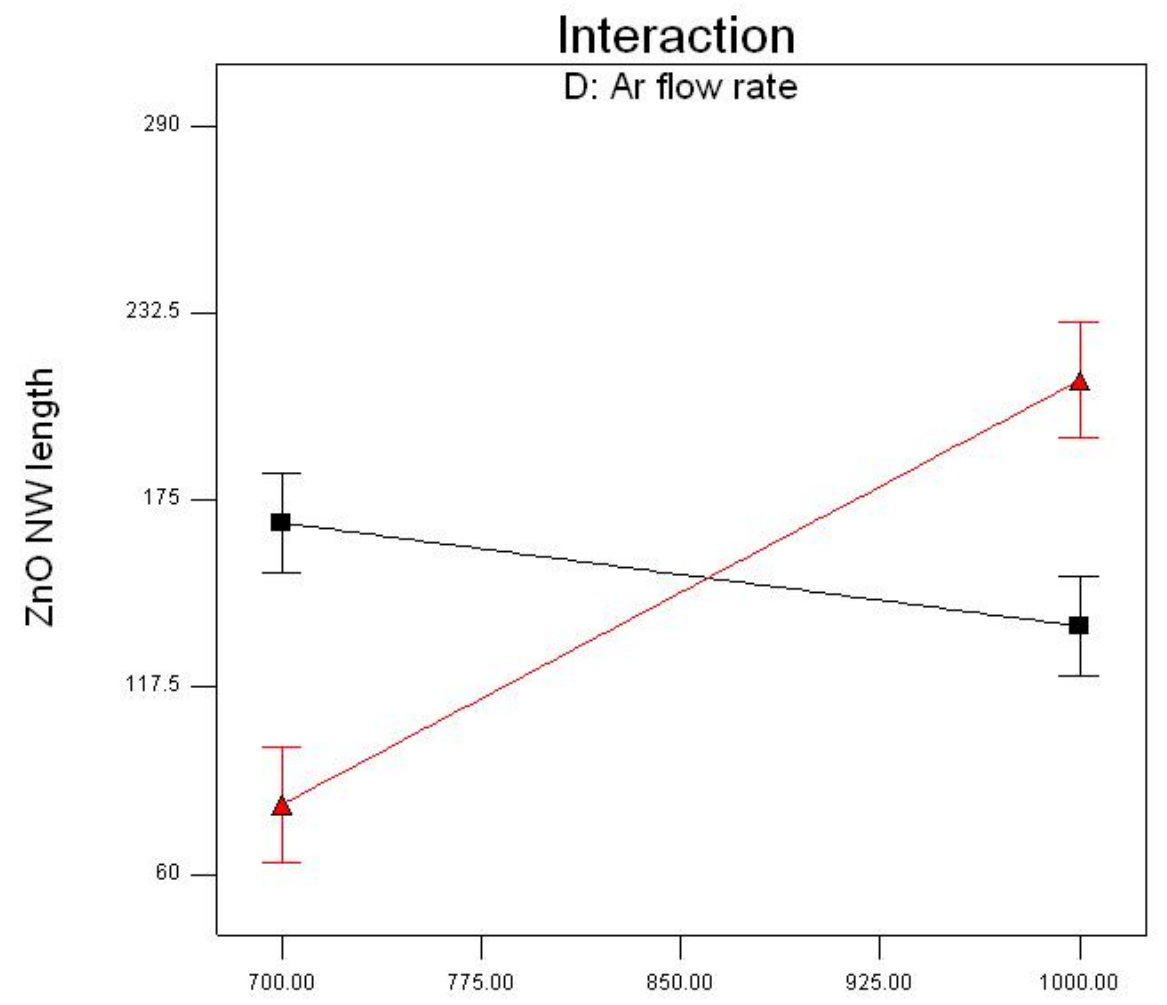

B: Temperature

Figure 8.4: Stat-Ease screenshot of graph of Temperature and Ar-flow. 
It helps in finding optimal values and helps the experimenter to understand which parameters will influence the output. The disadvantage is that the experimenter must choose the starting parameters and if he chooses wrongly, things will not work out as desired. 


\section{Chapter 9}

\section{Manufacturing of a $\mathrm{ZnO}$ nanowire pressure sensor}

\subsection{Introduction}

The main objective of this thesis was to grow $\mathrm{ZnO}$ nanowires and then use them in the manufacture of a pressure sensor. $\mathrm{ZnO}$ nanowires make this possible, because of the inherent piezoelectric effect. The growth of the $\mathrm{ZnO}$ nanowires was the most important step of the project, because if the nanowires could not be grown, no sensor could be manufactured.

The growth of $\mathrm{ZnO}$ nanowires is, however, well documented and a quick search for 'ZnO nanowires' using Scopus (an academic search engine) [111] returns nearly 2000 hits. Obtaining a method to grow the nanowires, however, was not that easy. There are many different methods of growing nanowires but only a few were viable with the equipment at hand. As described in Chapter 5, the VLS method was used to grow the $\mathrm{ZnO}$ nanowires.

Building the pressure sensor from the $\mathrm{ZnO}$ nanowires was a new application of the nanowires. To the author's knowledge, this is the first pressure sensor based on $\mathrm{ZnO}$ nanowires that has been built. Searching Scopus for 'zinc oxide nanowire pressure sensor' returned only 19 results, and only one of these had the word 'pressure sensor' in the title, although a operational concept sensor was not built.

The manufacture of the device was difficult, because it was a new idea. The growth of the nanowires was easier, because of the freely available methods, but the rest had to be done from scratch. The manufacturing of the pres- 
sure sensor comprised a number of steps, which are described below.

\subsection{Manufacturing}

The manufacturing of the pressure sensor was broken down into six steps:

1. Cutting $10 \times 10 \mathrm{~mm} \mathrm{Si} \mathrm{(100)} \mathrm{samples.}$

2. Drilling away an area for wirebonding.

3. Using photoresist to pattern a sample for a gold layer and an edge for glue.

4. Growth of $\mathrm{ZnO}$ nanowires on Si-Au (100) samples.

5. Gluing of two samples, with nanowires on them, together.

6. Adding wires to the device.

Figure 9.1 is a graphical representation of the manufacturing process. Each step is broken down into smaller steps to show how each step in the process is done.

\subsubsection{Sample cutting}

Large silicon wafers were used as a basis for the device. Silicon with a crystal configuration of (100) was used, simply because it is freely available and, according to some research, yields the best and most upright $\mathrm{ZnO}$ nanowires [51]. The wafers come in different sizes, all larger than $10 \times 10 \mathrm{~mm}$. The wafers used in this project had a diameter of $76.2 \mathrm{~mm}$ and a thickness of 350-400 $\mu \mathrm{m}$. These large wafers were then placed on the sample cutter and the samples were cut down to size. The samples were cut into $10 \times 10 \mathrm{~mm}$ squares, because most of the equipment in the laboratory was designed to work with these dimensions. Making the samples smaller raises another issue, namely handling and transporting of the samples. The cutting of the large wafer must be planned ahead of time in order to get the greatest number of samples possible from one wafer. With careful planning, more than 20 samples can be cut out of a single wafer. 
CHAPTER 9. MANUFACTURING OF A ZNO NANOWIRE PRESSURE SENSOB8

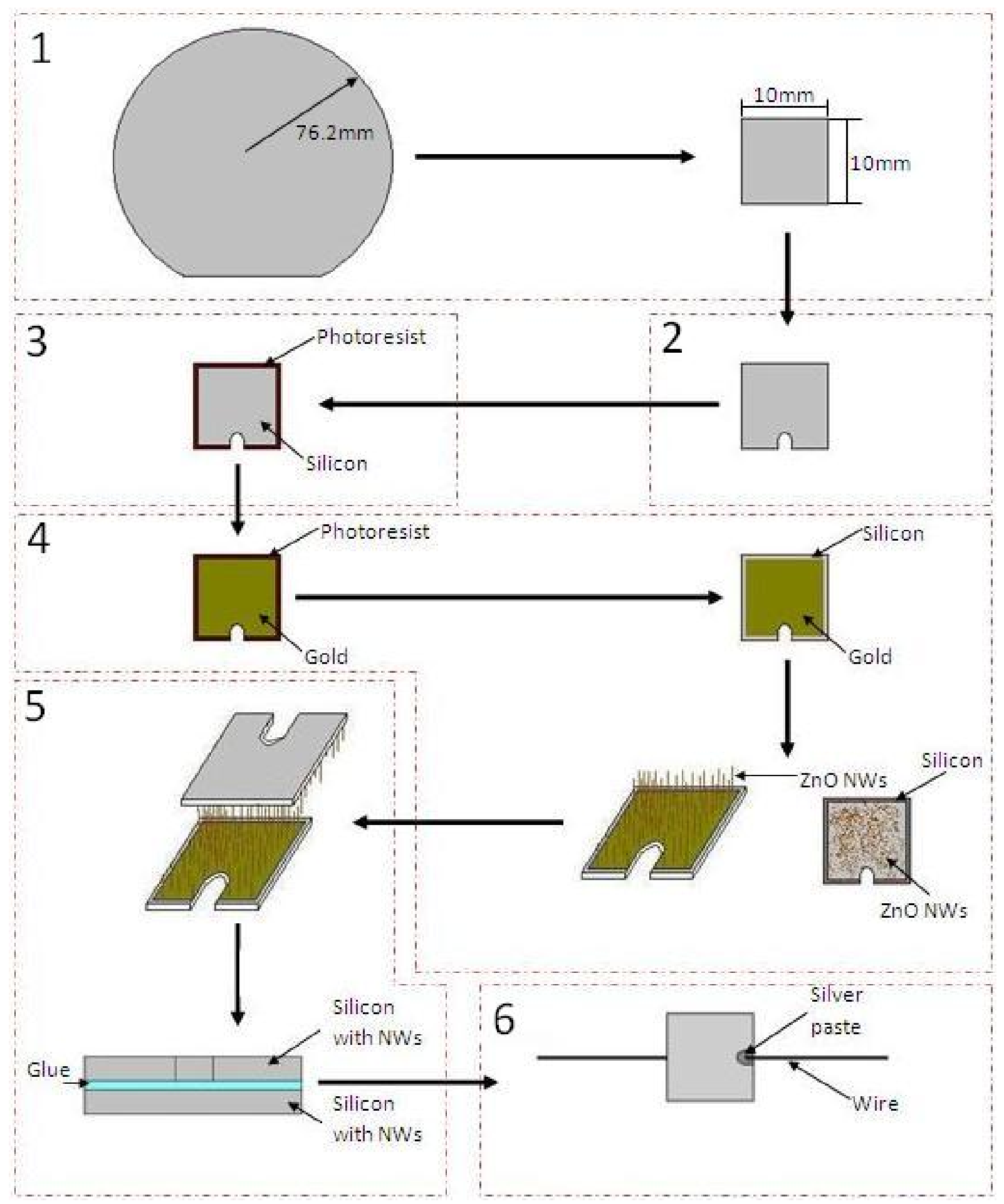

Figure 9.1: Schematic representation of manufacturing steps 


\subsubsection{Drilling}

The samples must be wirebonded at the end of the manufacturing process, and in order for that to be done, contact points must be found. The easiest way to ensure good contact points for the wirebonding, is to make a notch in the silicon sample. Like glass, silicon is a fragile material. Using a normal drill to make a notch in the silicon will not work, because the silicon will just crack and shatter. The Sonic Mill makes it possible to 'drill' a notch, as desired. The tip used in the sonic mill was chosen as $3 \times 3 \mathrm{~mm}$ square, with round edges. The round end was added, because a normal square hole made the edges too weak and the sample then broke easily.

\subsubsection{Pattern}

The sample was now the right size and had a hole for wirebonding. The next step was to split the sample into two regions. The centre of the sample was used to grow the $\mathrm{ZnO}$ nanowires. The border of the sample was used to put glue on, in order to glue two samples together.

The pattern was made via a photoresist and development process. S1818 photoresist was spun onto each sample at $3000 \mathrm{rpm}$ for 30 seconds. Each sample was then pre-baked, placed on a hotplate to harden the photoresist, at $115^{\circ} \mathrm{C}$ for 90 seconds. Using the lithography machine, a pattern was made on the photoresist. The pattern was $10 \times 10 \mathrm{~mm}$, with a border of $1 \mathrm{~mm}$ and a square of $8 \mathrm{~mm}$ by $8 \mathrm{~mm}$ in the centre. The lithography machine shines UV light through the pattern, with the centre piece open and the edge blocking the light. S1818 is a positive photoresist, and when developing the sample, the area where the light shone will be removed. After lithography the sample had a border of $1 \mathrm{~mm}$ around it and a clean $8 \mathrm{~mm}$ by $\mathrm{mm}$ square in the centre. The notch in the silicon did not have an edge around it.

\subsubsection{Growth of $\mathrm{ZnO}$ nanowires}

The growth of the $\mathrm{ZnO}$ nanowires was the most crucial part of the project. In Chapter 5 all the different methods for growing the nanowires were discussed. As stated there, the VLS method was decided upon to grow the nanowires.

The $10 \times 10 \mathrm{~mm}$ samples, now with a notch and with a border pattern on it, was put in the gold sputter unit. A thin layer of gold was deposited onto 
the entire sample. The gold layer was typically 15-20 nm thick. After the gold layer had been deposited, the sample was put into acetone to remove the photoresist around the border of the sample. Gold was deposited on top of the photoresist, but when the sample was placed in acetone, the photoresist dissolved. The gold attached to the photoresist was thus also removed from the sample. The sample now had an $8 \times 8 \mathrm{~mm}$ layer of gold, with a clean $1 \mathrm{~mm}$ border. Figure 9.2 shows the gold layer on the Si substrate. In the figure the darker area to the left is the deposited gold layer.

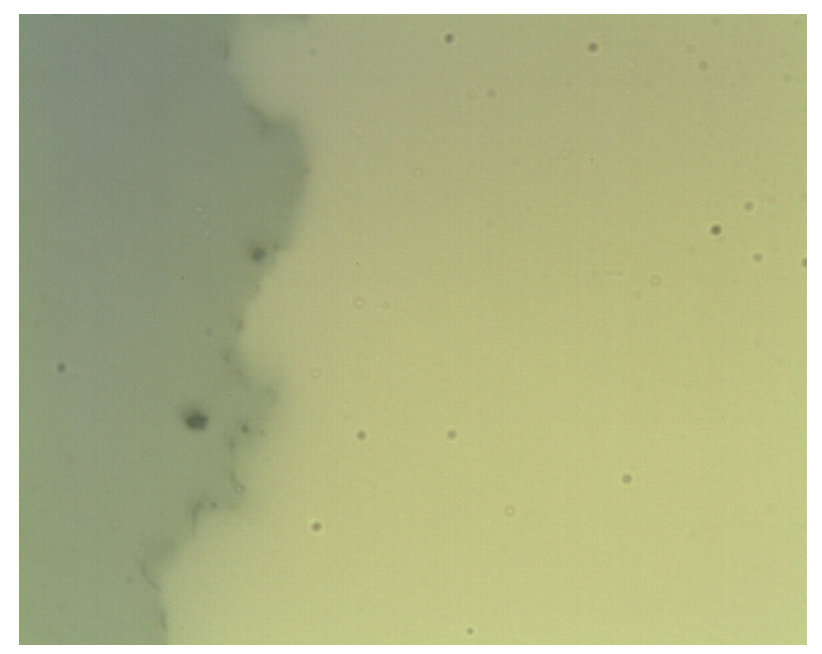

Figure 9.2: Si substrate with layer of gold deposited on top.

The sample was then placed inside a stainless steel tube, that was placed inside a furnace, to anneal the gold. This process involved heating up the sample in order for the gold atoms to break their strong bonds and form clusters of gold. The thin layer of gold was thus transformed to small, nanosized, droplets of gold. The stainless steel tube was closed at both ends, with only a small opening to pass gas through. Argon gas was passed through the system continuously. Figure 9.3 shows the gold layer after the annealing process. The picture looks different from Figure 9.2 and it is clear that the the layer is no longer continuous.

After that, a ceramic boat with a 1:1 mass mixture of $\mathrm{ZnO}$ powder and graphite powder was also loaded into the tube. The ceramic boat was placed in the centre of the furnace, with the sample placed $80 \mathrm{~mm}$ from the edge of the ceramic boat. This means that the sample was about $100 \mathrm{~mm}$ from the 


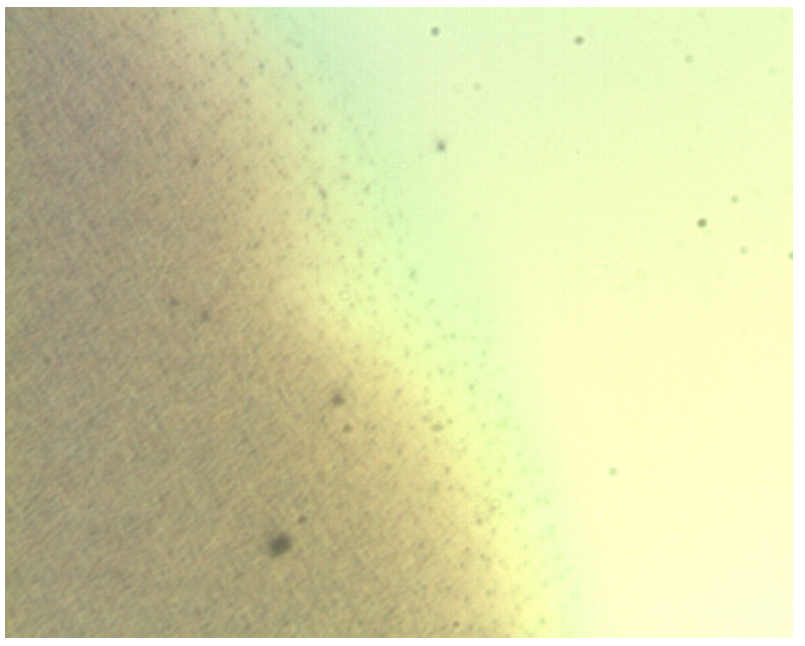

Figure 9.3: Si substrate with annealed gold layer.

centre of the furnace. The furnace was then heated up to different temperatures, ranging from $700^{\circ} \mathrm{C}$ to $1000^{\circ} \mathrm{C}$, in order to grow the nanowires. The argon gas flowed at a steady rate of either $60 \mathrm{sccm}$ or $120 \mathrm{sccm}$, depending on the experiment. Chapter 8 describes the reasoning behind the gap between the ceramic boat and the sample, as well as the reason for the exact gas flow rate.

The sample was left at the high temperature for either 10 minutes or 40 minutes. The system was then left overnight to cool down to room temperature. The sample was then removed from the tube and inspected via an optical microscope, as well as an AFM. The height of the nanowires was measured and the density was observed. It is difficult to determine the width of the nanowires, because they are wide at the root, a few hundred nanometers, while the tip is only a couple of nanometers, $10-80 \mathrm{~nm}$.

Professor Z.L. Wang at the Georgia Institute of Technology is one of the most cited scientists when it comes to $\mathrm{ZnO}$ nanowires [112]. Growing the nanowires was the most important step in the manufacturing of the pressure sensor, and because it is such a crucial step, a sample was imported from Professor Wang to ensure that the nanowires grown at Stellenbosch looked the same as his. Figure 9.4 shows the sample from Professor Wang, as scanned with the AFM system.

The scan shown in Figure 9.4 looks very similar to the scans of $\mathrm{ZnO}$ nanowires grown at Stellenbosch. Unfortunately the sample was never taken 


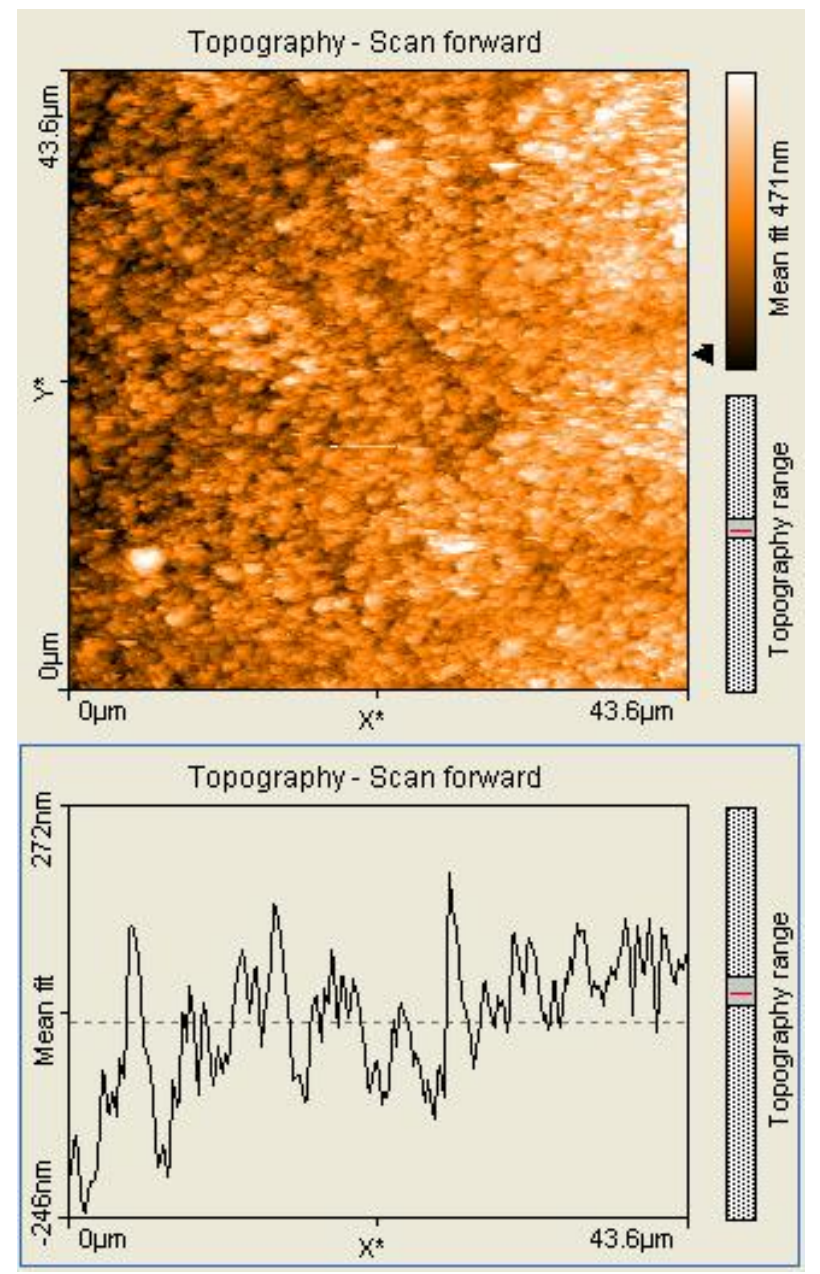

Figure 9.4: $\mathrm{ZnO}$ nanowire sample from Professor Wang at Georgia Institute of Technology.

to the SEM, because it was glued to a glass surface that was too big to fit into the SEM. Removing it from the glass surface would have damaged the sample.

\subsubsection{Gluing of two samples}

The penultimate step in manufacturing of the device was to glue two samples together. The samples were glued in such a manner that the nanowires were facing each other. The samples need to be able to move a bit for the nanowires to touch and bend each other. The glue must allow for this slight movement, 
but still be strong enough to hold the two samples together. Because the edge of the samples were still clean silicon, a silicon based glue was required, as normal glue does not stick to silicon. Silicone sealant from MAXFIX was used. It is a glue that is used to waterproof edges around basins.

The device now comprised of two silicon samples, with a layer of glue between them. The thickness of the glue layer must be more than the height of the nanowires. The nanowires are typically around $300 \mathrm{~nm}$ high, which means that the glue must be around $500 \mathrm{~nm}$ thick. Supports were made from the same silicon wafers to ensure that the thickness of the silicon remained the same. Photoresist was spun onto a silicon sample, at the right speed (about $8000 \mathrm{rpm}$ ), to make it $500 \mathrm{~nm}$ thick. The supports were placed on either side of the sample. Glue was then placed on the border of the sample, using a needle, and the second sample was placed on top of the glue, with the nanowires facing downwards. A level glass plate was put on top of the two samples, resting on the supports on either side. A weight was then placed on the glass plate. This ensured that the glue would spread around, but that the thickness of the glue layer would remain the same as that of the layer of photoresist, around $500 \mathrm{~nm}$. The setup was left for 24 hours to set properly.

\subsubsection{Wirebonding}

The last step in the manufacturing process of the device was to add wires where the notches had been drilled. One of the samples had to be coated with gold again, after the nanowire growth, because, as described in Chapter 4 , the electron affinity and the work function values had to be different in order to form a Schottky contact. Both samples had a gold layer, which is conductive, and the zinc oxide is also conductive. The notches in the samples thus made it possible to make simple contacts on the $\mathrm{ZnO}$ nanowires, and the $\mathrm{ZnO}$ nanowires covered in gold. Conductive silver paste was used to add wires to the area exposed by the notches. This was done on both sides. The silver paste was left for 24 hours to harden.

\subsection{Manufacturing difficulties}

Each of the steps described above was performed for each device that was built (except for the cutting of the large wafer). Each step had its own set 
of problems that had to be overcome in order to manufacture a operational device.

\subsubsection{Sample cutting}

Cutting the large wafer into the $10 \times 10 \mathrm{~mm}$ samples was by far the easiest step. However, it did present one problem. The tool used for cutting has a sharp diamond-like tip. The tip did not cut the wafer all the way through, but only made a trench, along which the wafer is then most likely to break. This was not always the case, sometimes the wafer would break in other places. This breakage meant that fewer samples could be made from a specific wafer.

\subsubsection{Drilling}

Drilling the notches into the samples was done via a Sonic Mill. The Sonic Mill, as described in Chapter 6, uses a special mixture of water and an abrasive material to perform the drill-like action. If there was too much water, and not enough abrasive material, the sample would simply crack and break into pieces. If the mill moved too fast, the sample would also break. The correct speed and level of material had to be found to ensure that the samples did not break. The speed was set very slow to ensure the correct operation, which meant it was a time consuming task to drill the holes.

\subsubsection{Pattern}

Applying the pattern to the sample was easy. Spinning on photoresist is a simple task. Using the lithography machine was also easy. One must just ensure that the mask and the pattern is lined up as desired. The right developer must then be used. This information is given in the datasheet of the photoresist.

\subsubsection{Growth of $\mathrm{ZnO}$ nanowires}

The growth of the nanowires took the longest to master. In the literature growth procedures were performed on special equipment, and all the details were not always given. Optimisation of the gas flow rate, the distance between the sample and the ceramic, and the temperature, were all necessary. 
All three of these parameters are dependent on one another, as described in Chapter 8.

\subsubsection{Gluing of two samples}

The technique used to glue the two samples together was a quick solution to a big problem. Adding a layer of glue only a few hundred nanometers thick is more difficult than at first presumed. There is no scale that can measure so thin a layer and specialized equipment was out of the question, due to the high costs. The technique used would have worked well if the silicon wafer was of a uniform thickness. The manufacturer of the silicon wafer stated that the thickness is $350 \mu \mathrm{m}-400 \mu \mathrm{m}$. That gives a variance of $50 \mu \mathrm{m}$, which is large, because the nanowires are only $300 \mathrm{~nm}$ long. This presented a few problems, because the thickness of the supports on either side of the samples, and the samples themselves, with nanowire growth, had to be the same for this technique to work. Determining the thickness of each sample down to the micrometer was not possible, so an element of luck was involved in the gluing step.

More than 20 devices were made throughout the course of this project, of which only a couple worked as desired. The outcome of this step in the manufacturing process is the step most likely to have a large impact on the correct operation of the device.

\subsubsection{Wirebonding}

The conductive silver paste used for the wirebonding worked well. The wires attached to the samples did break off sometimes, and were then just glued back on with more silver paste.

\subsection{Conclusion}

The manufacture of the device was a daunting and time-consuming task. There was no literature available on appropriate methods to manufacture the device. Each of the steps took a long time to perform, and as a result each device took about five days to manufacture, beginning with a large wafer to the final product, a working device. 
CHAPTER 9. MANUFACTURING OF A ZNO NANOWIRE PRESSURE SENSQR6

As described above, some steps were accomplished more easily than others, with the gluing of the two samples the most difficult task, because of factors beyond the author's control. 


\section{Chapter 10}

\section{Measurements and Results}

\subsection{Introduction}

The creation of a $\mathrm{ZnO}$ nanowire based pressure sensor had two core parts, namely manufacturing and testing. The manufacturing of the nanowires has already been discussed in detail in Chapter 9. The last part of the project was to test the sensors, by measuring the relationship between the applied pressure and the output voltage of the sensor.

This first part of this chapter looks at results from the growth of $\mathrm{ZnO}$ nanowires. This is only a brief section and a more detailed description of the nanowires can be found in Chapters 1, 8 and 9. The next section examines the relationship between the applied pressure and the output voltage of the sensor. The chapter ends with a brief conclusion.

\subsection{Nanowire growth}

The growth of $\mathrm{ZnO}$ nanowires has already been discussed in detail throughout Chapters 5, 8 and 9. This section is included to give a brief report on the results obtained during the growth of the nanowires.

The nanowire growth for the manufacture of the pressure sensor was done with the parameters shown in Table 10.1. The parameters in Table 10.1 were chosen according to the results obtained from the Design of Experiments. These parameters resulted in the longest and densest nanowires. On average the nanowires were $300 \mathrm{~nm}$ in length, with a diameter of about 80-150 $\mathrm{nm}$, as seen in Figure 10.1. 
Table 10.1: Different parameters and their values as used for $\mathrm{ZnO}$ nanowire growth

\begin{tabular}{|c|c|}
\hline Parameter & Value \\
\hline System Temperature & $1000^{\circ} \mathrm{C}$ \\
\hline Growth Time & $40 \mathrm{~min}$ \\
\hline Argon flow rate & $60 \mathrm{sccm}$ \\
\hline Gold layer thickness & $20 \mathrm{~nm}$ \\
\hline
\end{tabular}

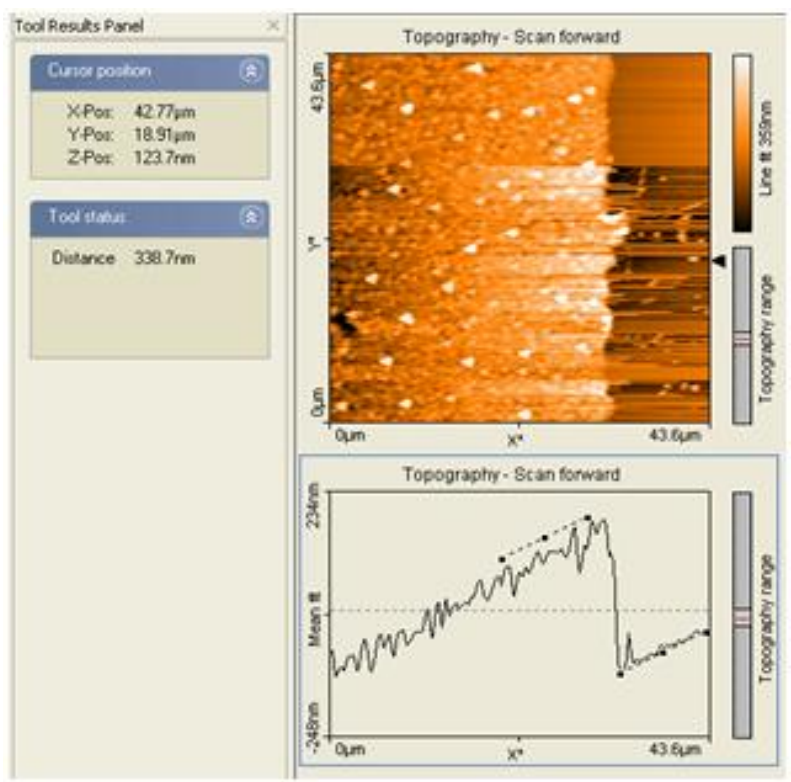

Figure 10.1: Typical nanowire growth as measured by AFM

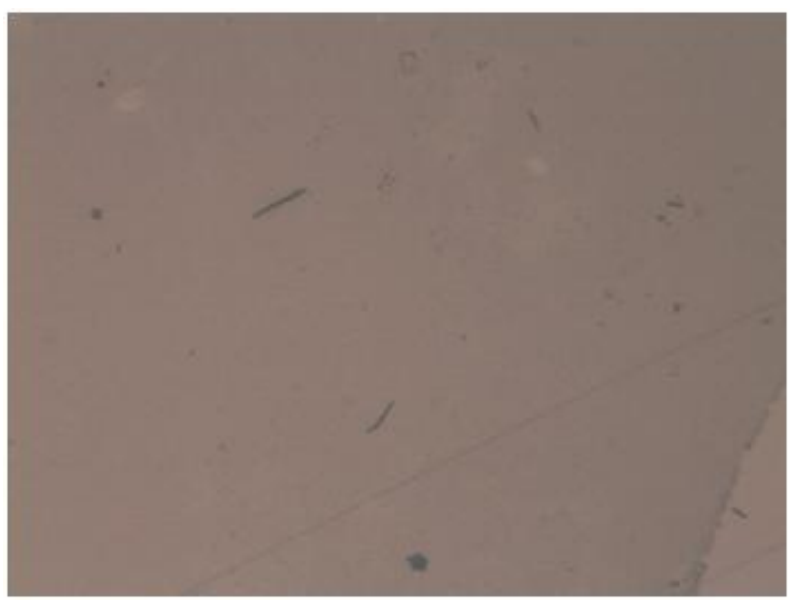

Figure 10.2: Typical nanowire growth picture taken by optical microscope 
Figure 10.2 is a picture taken with a normal optical microscope. In the bottom right corner a piece of clean silicon, with no grown nanowires on, can be seen. The rest of the sample has grown $\mathrm{ZnO}$ nanowires. This picture gives an indication of the density of the wires, albeit difficult to see. In Figure 10.1 the density can also be seen, where all the shapes on the left are nanowires.

After the growth of the nanowires, the manufacturing process, as described in the previous chapter, was followed to make the pressure sensor. Figure 10.3 shows the finished device. Measurements were made using this device and others that looked similar.

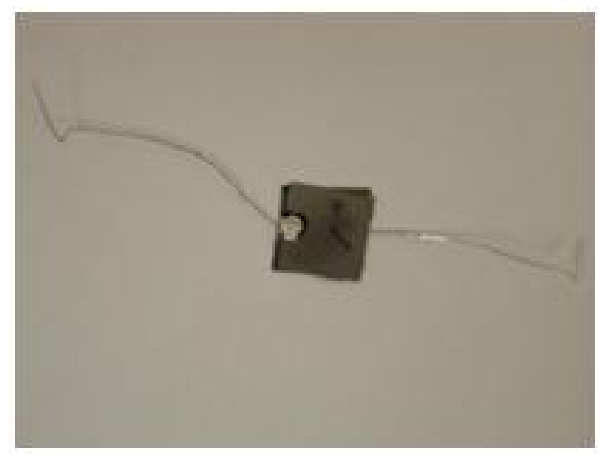

Figure 10.3: Picture of the finished sensor

\subsection{Pressure versus Voltage}

The $\mathrm{ZnO}$ nanowires exhibited the piezoelectric effect, as described in Chapter 4. In short, this means that when pressure is applied to the $\mathrm{ZnO}$ nanowires a voltage is generated, due to the piezoelectric effect. This is the basis for the operation of the pressure sensor.

The output voltage of the signal was too small to make accurate measurements. The signal was only a few millivolts, approaching ten millivolts at maximum. A simple amplifier was thus built. The amplifier had a gain of 1000. The measured output was now a few volts, and this made accurate measurements much easier. 


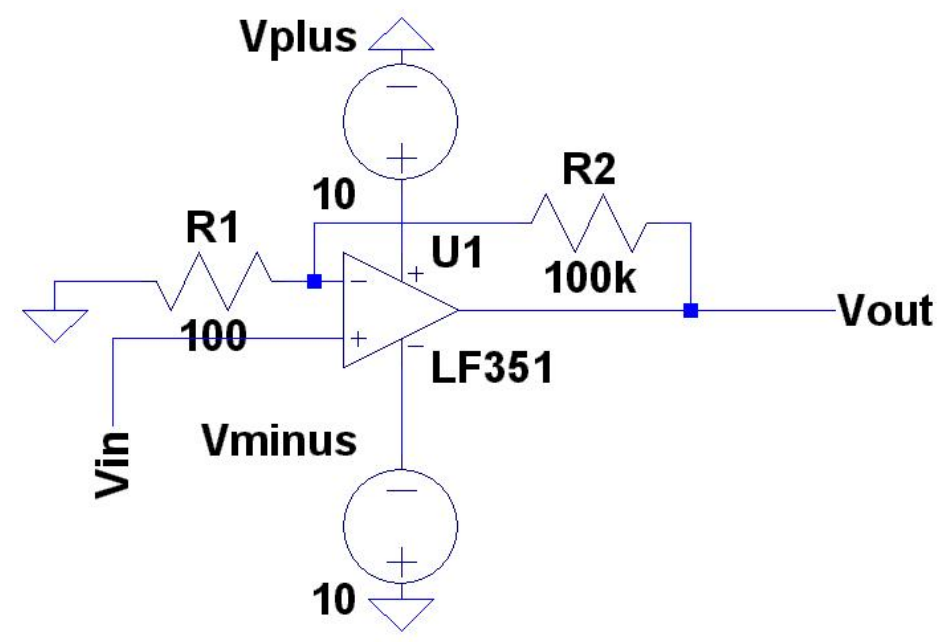

Figure 10.4: Circuit diagram of amplifier used to amplify voltage

The circuit shown in Figure 10.4 is the amplifier that was used to amplify the output voltage of the pressure sensor. The gain of the circuit is given by

$$
A_{v}=1+\frac{R_{2}}{R_{1}} \approx \frac{R_{2}}{R_{1}} \text {, when } \frac{R_{2}}{R_{1}}>>1 \text {. }
$$

For the circuit shown, the gain will be 1000. The pressure sensor is connected at the $\mathrm{V}_{\text {in }}$ terminal and the measurements are made at the $\mathrm{V}_{\text {out }}$ terminal. The measurements were made by a multimeter. For the first few runs the measurements were made on an oscilloscope. The data obtained from the oscilloscope was very noisy and did not add anything more than a multimeter measurement. Figure 10.5 shows a typical signal from the pressure sensor. The start is just noise and the peak occurs when pressure is applied. For a pressure sensor the exact voltage signal is not necessary, but the maximum value is of greater importance. The rest of the measurements were made with a multimeter. Pressure was applied and the maximum value was then taken.

The maximum value, rather than an average measurement, was taken, because the pressure sensor has capacitor-like behaviour. When pressure is applied to the sensor, the voltage shoots up to a maximum value and then slowly returns to zero, which is similar behaviour than a discharging capacitor. To take an average measurement, one must wait until the sensor output voltage returns to zero, which can take some time. 


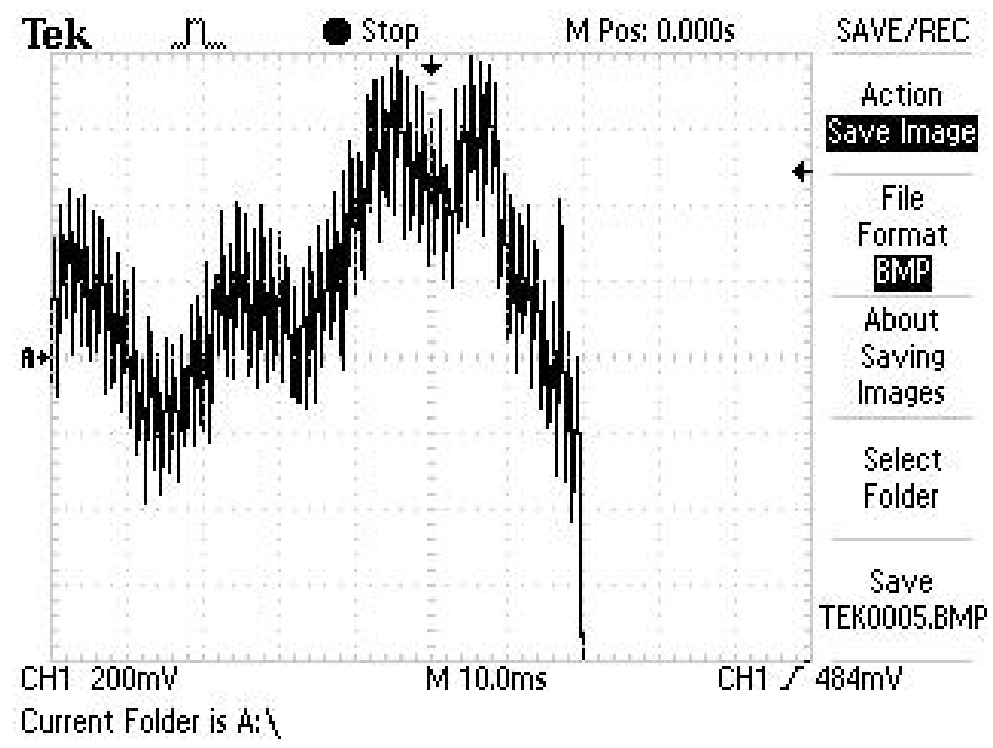

Figure 10.5: Output waveform of pressure sensor when pressure is applied

Measurements were made by putting different weights on the sensor. The weights were placed on a small $5 \mathrm{~mm}$ by $5 \mathrm{~mm}$ block. This made it easy to convert weight to pressure, as pressure is just the force applied on a certain area, as described in Chapter 3. The area in contact with the sensor remains the same $\left(25 \mathrm{~mm}^{2}\right)$ and only the weight changed for each measurement. Table 10.2 shows all the different weights used and the corresponding pressure applied to the sensor.

Table 10.2: Pressure exerted on sensor via different weights.

\begin{tabular}{|c|c|}
\hline Weight (g) & Pressure (Pa) \\
\hline 0.9 & 391.4 \\
\hline 7.7 & 3348.3 \\
\hline 10.5 & 4565.8 \\
\hline 11.1 & 4826.7 \\
\hline 23.5 & 10218.8 \\
\hline 38.8 & 16871.8 \\
\hline 64.7 & 28134.2 \\
\hline 147.5 & 64139.0 \\
\hline 295 & 172604.2 \\
\hline
\end{tabular}


The device is manufactured under atmospheric pressure, which means that the atmosphere does not exert any additional pressure on the device and may thus be ignored in measurements. If the device is taken to higher or lower altitude, the atmospheric pressure on the device would have to be taken into account.

Figure 10.6 shows the measured voltage versus applied pressure. The different lines represent different sensors. Six sensors were tested, as seen in Figure 10.6. More sensors were actually tested, but only six are shown in Figure 10.6. Figure 10.7 shows three more sensors, that were omitted from Figure 10.6 because the figure got too cluttered.

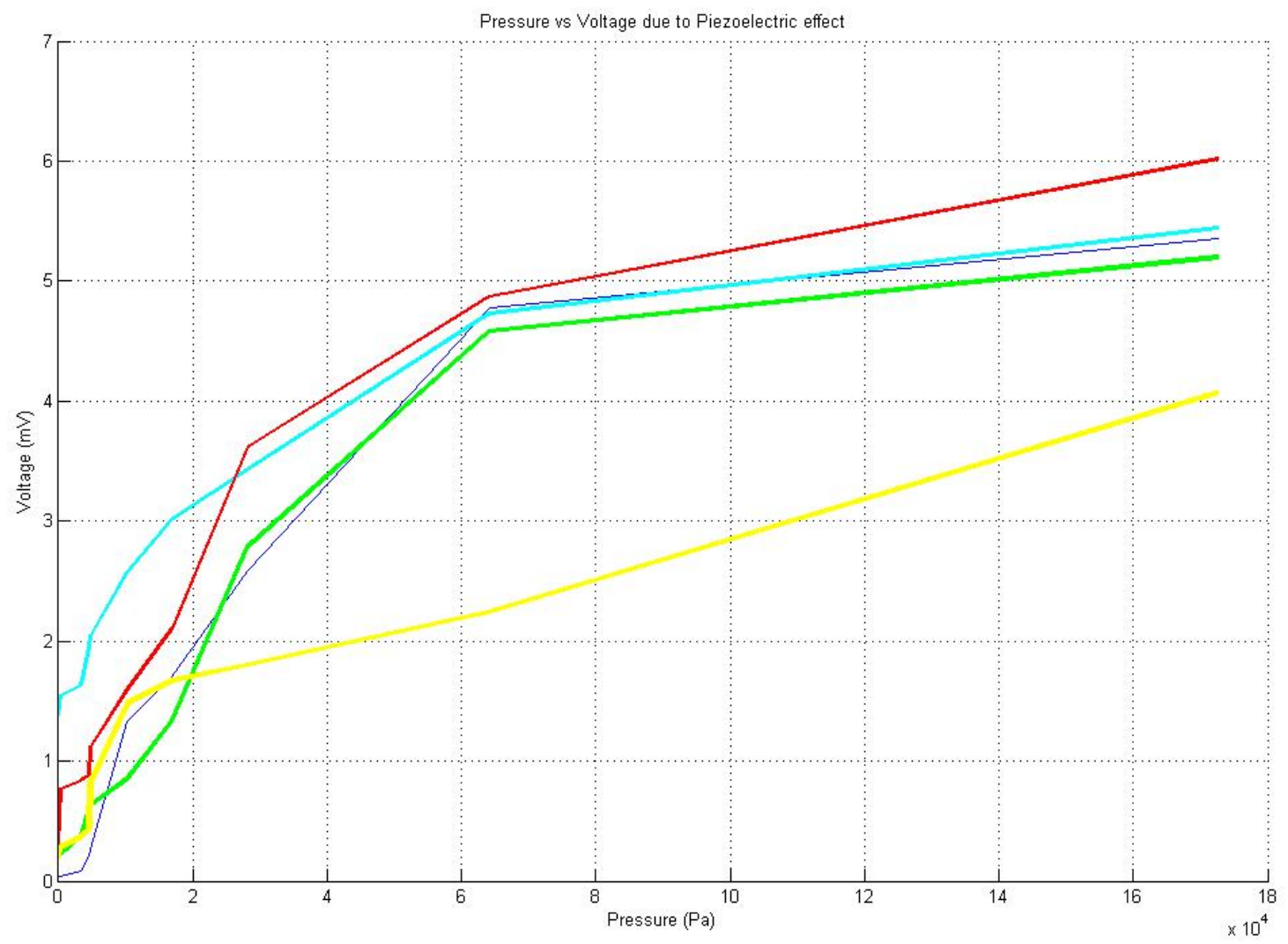

Figure 10.6: Voltage vs pressure graph

It is clear from the figure that most of the sensors exhibit the same behaviour. Only the yellow line differs from the other five. It is lower and 


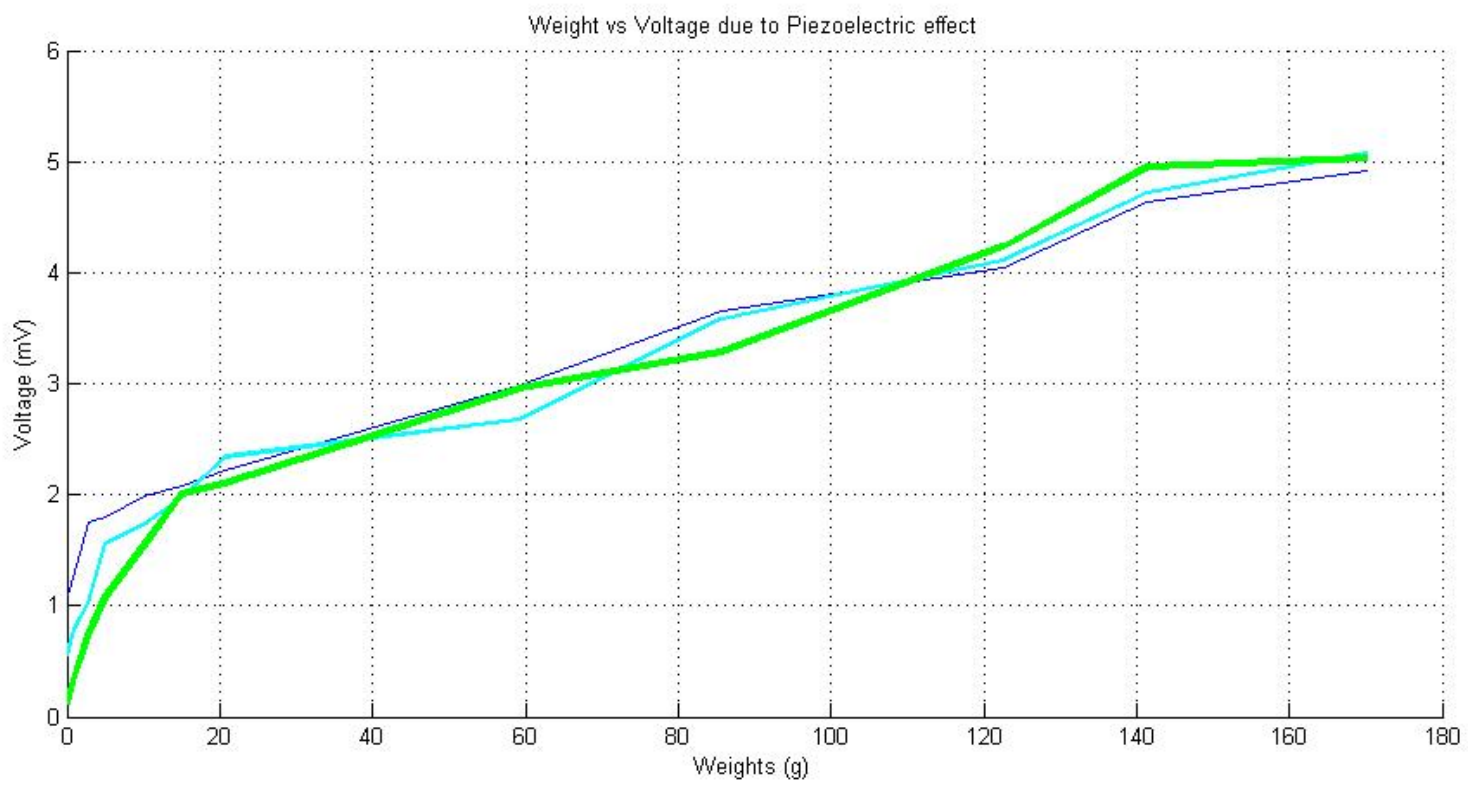

Figure 10.7: Voltage vs pressure graph for three extra sensors

increases more rapidly than the others. The other five sensors all show an almost linear increase up to $60 \mathrm{kPa}$ and then flatten out slightly and continue on, again in a linear fashion. This means that the sensor voltage rises quickly at the start, as the pressure rises, and then it flattens out at the end. Each sensor exhibits a maximum (saturation) value, and it converges to that maximum. The yellow line does not show this behaviour, but instead it continues to rise quickly. It could be that this sensor has a much higher saturation value and that it will only flatten out at a much higher pressure. Figure 10.6 and Figure 10.7 only shows behaviour of a selected number of sensors. Many more sensors were tested and exhibited the same behaviour as the devices shown in the figures. The figures shows only a few in order to illustrate the point, and adding more graphs would have cluttered the figures.

The sensors were also tested for the superposition principle, i.e. when two sensors are connected in series, the resulting voltage is expected to be the sum of the individual sensor voltages. The measured superposition voltage was never exactly the same as the sum of the two individual voltages, but it was in the same range. The reason for the inaccuracy can be ascribed to the fact that the sensors are heavily dependent on the position where the pressure is applied, and it was difficult to get exactly the same position every 
time. Table 10.3 shows results of the superposition tests for two different sets of sensors. Both times the superposition voltage is within 10 percent of the summed output voltages.

Table 10.3: Superposition of output voltage.

\begin{tabular}{|c|c|}
\hline Sensor & voltage $\mathbf{( m V )}$ \\
\hline 2 & 1.80 \\
\hline 3 & 1.56 \\
\hline $2+3$ & 3.09 \\
\hline 2 & 1.73 \\
\hline 8 & 1.53 \\
\hline $2+8$ & 3.06 \\
\hline
\end{tabular}

When a sensor is manufactured, one sample is coated with gold before the two samples are glued together. This is done in order to create a Schottky contact, as described in Chapter 4. In order to test the Schottky principle, a sensor was made with neither one of the samples covered with gold. When pressure was applied to that sensor, no output voltage was measured, which proved the theory that a Schottky contact must be present for the piezoelectric effect to be measurable.

The sensors are very sensitive to where the pressure is applied. As mentioned above, a small block was placed on the sensor, on a specific area. The weights were then placed on top of the small block to ensure that the force always acted on the same area. The output voltage varied a lot when the block was moved around on the sample. Figure 10.8 and Table 10.4 shows the measured output voltage for one sensor when the pressure is applied on different areas on the sensor.

Table 10.4: Output voltage of sensor with pressure applied on different areas of sensor.

\begin{tabular}{|c|c|c|c|c|c|c|c|c|c|c|c|c|}
\hline Posistion on sensor & $\mathbf{0 ~ g}$ & $\mathbf{0 . 9} \mathbf{g}$ & $\mathbf{2 . 8} \mathbf{g}$ & $\mathbf{5} \mathbf{g}$ & $\mathbf{1 0} \mathbf{g}$ & $\mathbf{1 5 . 1} \mathbf{g}$ & $\mathbf{2 0 . 7} \mathbf{g}$ & $\mathbf{5 9 . 3} \mathbf{g}$ & $\mathbf{8 5 . 6} \mathbf{~ g}$ & $\mathbf{1 2 2 . 5} \mathbf{g}$ & $\mathbf{1 4 1 . 4} \mathbf{g}$ & $\mathbf{1 7 0 . 1} \mathbf{~ g}$ \\
\hline Middle $(\mathrm{mV})$ & 0.76 & 0.79 & 1.02 & 1.56 & 1.73 & 1.98 & 2.34 & 2.68 & 3.58 & 4.11 & 4.72 & 5.08 \\
\hline Top Left $(\mathrm{mV})$ & 0.76 & 1.29 & 1.63 & 1.92 & 2.08 & 2.52 & 2.57 & 3.44 & 3.78 & 3.97 & 4.13 & 4.88 \\
\hline Top Right $(\mathrm{mV})$ & 0.77 & 1.31 & 1.63 & 2.08 & 2.47 & 3.06 & 3.53 & 4.14 & 4.77 & 6.66 & 6.76 & 7.40 \\
\hline Bottom Right $(\mathrm{mV})$ & 0.77 & 0.78 & 0.84 & 1.04 & 1.22 & 1.36 & 1.59 & 1.79 & 1.98 & 2.27 & 2.36 & 2.49 \\
\hline Bottom Left $(\mathrm{mV})$ & 0.76 & 0.78 & 0.80 & 0.80 & 0.82 & 0.85 & 0.91 & 0.93 & 1.12 & 1.30 & 1.36 & 1.48 \\
\hline
\end{tabular}




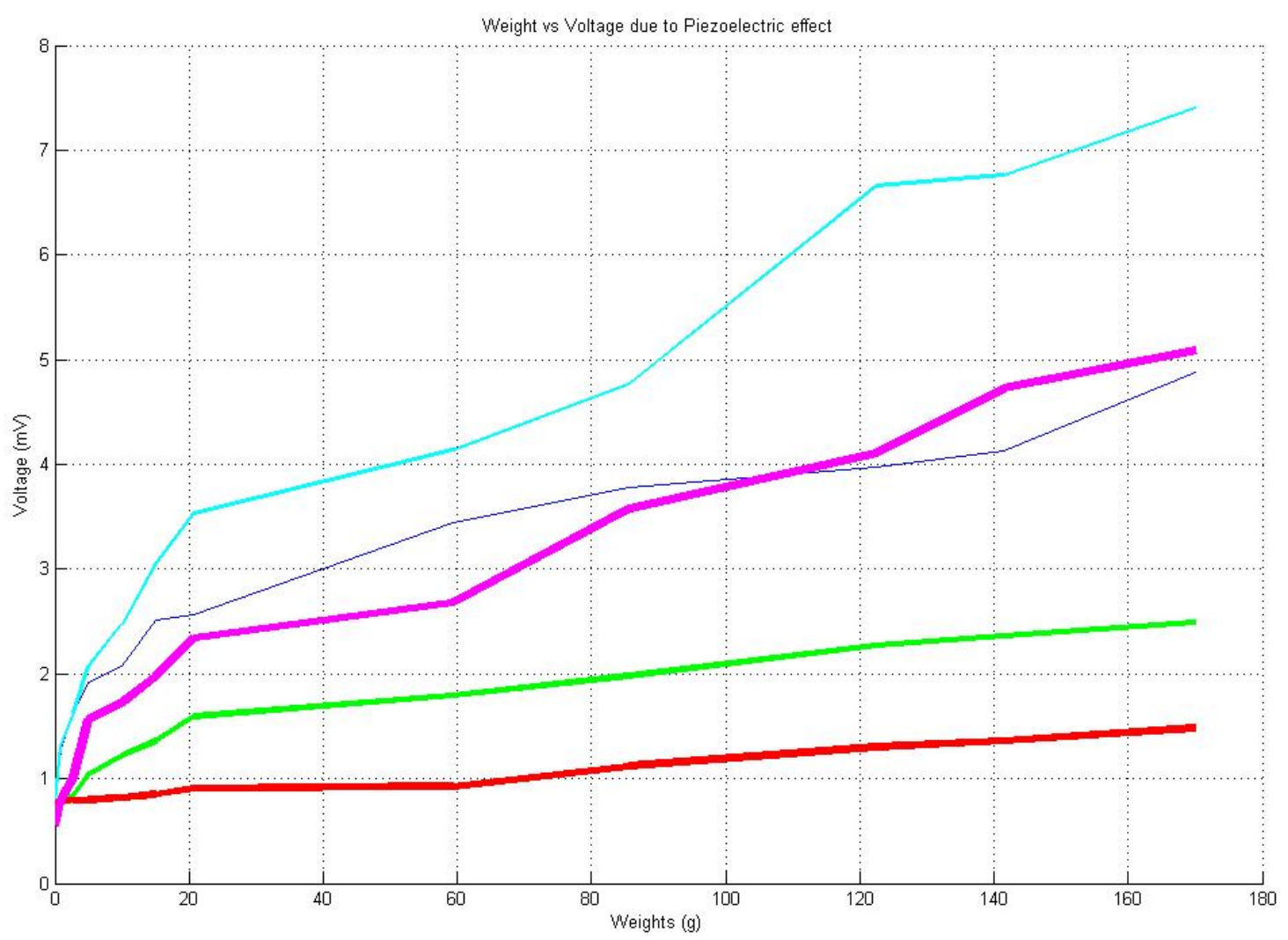

Figure 10.8: Output voltage of sensor when pressure is applied at different locations on the sensor.

From Figure 10.8 and Table 10.4 it is clear that the top side of the sensor yielded much higher voltages than the bottom side. The silver paste used for the wirebonding was applied over a wide area on the top side of the sensor. Some of the silver paste went in between the sensors. On the bottom side, the silver paste was applied only on the centre and no silver paste went in between the sensors. This made it clear that the silver paste had a major effect on the output voltage. $\mathrm{ZnO}$ is conductive, but with a resistance of over 30 kilo-ohms for a $10 \times 10 \mathrm{~mm}$ area. Silver, on the other hand, is a good conductor with a very low resistance. The top side, where the silver paste went in between the samples, had a higher voltage because the internal losses were less.

The $\mathrm{ZnO}$ and silver-paste can be viewed as two resistors in series. The current travels throught the $\mathrm{ZnO}$ resistor and then through the silver-paste 
resistor. The current through the bottom contact has to travel a longer distance through the $\mathrm{ZnO}$ to reach the wire connected with the silver paste, which means the $\mathrm{ZnO}$ resistor is much larger than the silver-paste resistor. The current encounters more resistance along the way, and the output is thus much lower because there is a large voltage drop across the $\mathrm{ZnO}$ resistor. On the top, the silver paste has moved into the sensor and the current has a shorter distance to travel through the $\mathrm{ZnO}$. The current travels longer through the silver, which has a lower resistance than that of $\mathrm{ZnO}$ and thus the losses are less. The losses are lower and thus the output voltage is higher. One of the sensors gave over $10 \mathrm{mV}$ when the pressure was applied at the end where the silver paste had been applied, and had gone in between the sensors. The output voltage almost doubled with the same applied pressure. The dependency of the results on the area where the pressure is applied, is directly related to the silver paste. When the silver paste came between the two samples forming the sensor, the output voltage was much higher.

The theory above was tested with a sample where the silver paste did not move in between the top and bottom samples. The voltage stayed very much the same, independent of the position where the pressure was applied. The voltage did, however, change to a degree and this is attributed to more nanowires being active in one region than in another.

To maximize the output voltage a good conductive layer must be present on both samples that make up the sensor. One sample is covered with gold to ensure the Schottky contact. The other sample must also have a conductive layer to ensure good conductivity, but also to ensure that the Schottky contact will form.

All the tests confirmed that the voltage that is being measured is indeed as a result of the piezoelectric effect and not just noise or other system voltages. The superposition results clearly show that the voltage comes from the sensors. The theory of the Schottky contact was also proven, and it was observed that when the gold layer was not present, no voltage was measured. The dependency of results on the area where the voltage was applied, came down to the silver paste used for wirebonding. When the silver paste went in between the two samples forming the device, the output voltage was much higher. This also showed that the voltage can be increased by lowering the resistance of the sensor.

Experiments done on bulk materials that exhibit the piezoelectric effect 
showed similar behaviour as seen in Figure 10.6 [113]. Force sensing resistors (FSR) also have the same behaviour as seen in Figure 10.6 [114]. The ZnO nanowire pressure sensor thus exhibited similar behavioural characteristics to bulk material and other force sensors. The main disadvantage at this stage is that the nanowires generate a much lower voltage than bulk material. Bulk material can give hundreds of volts if a large force is applied [113]. To make the $\mathrm{ZnO}$ nanowire sensor more efficient, the output voltage should thus be increased.

The repeatability of the voltage measurements always stayed within $10 \%$. In other words, if the voltage was measured at $5 \mathrm{mV}$ at $80 \mathrm{kPa}$, a measurement taken a few hours or a few days later would not differ by more than $10 \%$. The measurements were very precise, with little to no variation shown between separate measurements. According to the mathematics in Chapter 4, the output voltage is equal to the voltage of only one nanowire. The nanowire with the maximum voltage thus determines the output voltage. This means that, as the pressure increases, more nanowires make contact with each other and more bending occurs. The nanowire with the highest potential difference will change and as a consequence the output voltage will change. A higher pressure will seldom lead to a lower voltage, because there are millions of nanowires in contact, and at least one will have a higher voltage than what was measured in the previous case with lower pressure. This makes the measured voltage an excellent parameter to indicate that pressure has been applied to a device.

The voltage between different devices did vary to some degree. In Figure 10.6 the light blue and dark blue lines are very close to each other. The green line is just beneath these two, and the red line just above, with only the yellow line being completely different. This means that, for each device, different voltages will be measured for the same applied pressure. If the yellow line is omitted from the results, at $100 \mathrm{kPa}$ the mean voltage is almost $5.03 \mathrm{mV}$. The two blues lines are at $5 \mathrm{mV}$ at $100 \mathrm{kPa}$, but the red line is already at $5 \mathrm{mV}$ at a pressure of $78 \mathrm{kPa}$. The green line only reaches $5 \mathrm{mV}$ at $140 \mathrm{kPa}$. Each device must thus be calibrated individually to ensure that it gives the correct voltage at the corresponding pressure. Taking the mean value at a certain pressure will not give accurate results.

The simulations, as well as the mathematics, show that the output voltage should be in the range of a few volts, and not millivolts. Looking at the 
simulation results in Chapter 7, a voltage of $0.358 \mathrm{~V}$ is measured when the nanowire is of the same dimensions as the nanowires used in practice. The pressure applied is also in the same range, but the voltage is much less for the practical nanowires. Even when the applied pressure is much more than that in the simulation, the voltage is still far lower. The reason is that, in reality, the device losses are not accounted for in the simulations. These losses are caused by resistance between contacts, as well as capacitance that is present in the device, and include:

1. Contact resistance between nanowires.

2. Resistance of the nanowires itself.

3. Resistance between the nanowires and the $\mathrm{Si} / \mathrm{Au}$ substrate.

4. Resistance of the $\mathrm{Si} / \mathrm{Au}$ substrate that transports the signal to the wires.

5. Resistance of the contact pads for wirebonding.

6. Resistance of the wires used for wirebonding.

7. Capacitance between nanowires.

8. Capacitance between the two Si/Au substrates.

In practice the mentioned losses will be present at all times, and the measured output signal will thus always be less, when compared to the ideal case.

\subsection{Current Measurements}

The output current of the device was also measured. The current is extremely low, in the range of a few pico-Amperes (pA), and conventional current measuring devices are not capable of measuring currents of that magnitude. A specialized pA-meter was used for the measurements. The meter is almost 30 years old and had not been used for the last couple of years. No online support is available, because the product is obsolete.

The measurements showed that current did also change with an increase in pressure, but not consistently. Each device gave a different looking graph, with values far from one another. Currents as high as a few micro-Amperes 
were measured at one stage. On a second occasion, all the devices gave identical readings. The readings, however, did not make much sense, because they would sometimes increase with an increase in pressure, but then drop drastically when the pressure is increased further. All the current measurements were in the order of $1 \mathrm{pA}$. The changes in current with an increase in pressure varied around $1 \mathrm{pA}$.

The current measurements did not make sense. According to the mathematics in Chapter 4, the current is the sum of all the individual currents through the active nanowires. When pressure is applied to the device, more and more nanowires should be in contact, which means the current should increase. This was, however, not the case. The only explanation is that, when the pressure increases, it reaches a maximum point where the most nanowires are in contact. When the pressure is increased further, the nanowires lose their contact and the current drops. Certain pressures cause more nanowires to make contact than others. Current can thus not be used as a measuring unit for pressure, because a higher pressure does not necessarily mean higher current. Current measurements were thus ignored, because of the lack of consistency.

The output power of the device is not great. The output voltage is a few millivolts and the output current a few pico-Amperes, which means that the output power is only a few femto-Watts. That is very low and will not be enough to power any electronic product.

\subsection{Conclusions}

A pressure sensor based on $\mathrm{ZnO}$ nanowires does show promise. For relatively low pressures (below $60 \mathrm{kPa}$ ) a small change in pressure results in a large increase in voltage. For higher pressures (above $60 \mathrm{kPa}$ ) the change in voltage is not so drastic, but is still noticeable. The pressure sensor will not measure pressures much higher than mentioned here, because the silicon substrate will break when the applied pressure approaches $200 \mathrm{kPa}$ to 250 $\mathrm{kPa}$. To ensure that the substrate did not break, all measurements were performed below the maximum level. Different substrates should be considered if higher pressure measurements are required.

The results obtained are in compliance with those of bulk material. The bulk materials show the same behaviour as the nanowires, but generated 
voltages are just much higher. The output voltage for the nanowire case is so low that it requires amplification in order to make reliable measurements. This makes the device bulky, as it still requires an external source to drive the amplifier. If the output voltage can be increased to a few volts, the device would be able to work independently of any other equipment.

The basis for a pressure sensor based on $\mathrm{ZnO}$ nanowires has been laid. The measurements are very promising and show that the sensor will work in practice. Improvements must be made on the substrate material to handle higher pressures, and the output voltage must be increased to make the sensor viable for industrial use. 


\section{Chapter 11}

\section{Conclusions}

Pressure has been measured for many years, going right back to Galileo Galilei in 1594. Nanotechnology is a very new field that started only in 1959 with Richard Feynman's talk "There is plenty of room at the bottom". Combining the two fields of pressure measurement and nanotechnology, in order to create a pressure sensor based on nanowires, presented a number of unique challenges.

The piezoelectric effect on which the sensor is based, narrowed the choice of material down, with zinc oxide being decided upon. The growth of the $\mathrm{ZnO}$ nanowires was a major part of making the sensor, because without them no sensor is possible. Of all the different methods that were investigated the vapour-liquid-solid (VLS) method yielded the best nanowires, and the VLS method was thus chosen for the growth of the nanowires that formed the basis of the sensor. The main problem with the VLS method is the high temperature at which the nanowires are grown, and at $1000^{\circ} \mathrm{C}$ a lot of energy is used. In most of the other methods investigated the synthesis of the nanowires was performed at much lower temperatures. Lower temperatures are desireable, because, for mass reproduction, it is economically cheaper to work at lower energy levels. The VLS method, however, gave the best results. More than 150 samples of $\mathrm{ZnO}$ nanowires were grown. All the samples were grown on $10 \mathrm{~mm} \times 10 \mathrm{~mm}$ silicon substrates. From these 150 samples it was clear that the VLS method was the only method that consistently produced good results.

Building the pressure sensor from scratch was challenging, as it was a completely new use for $\mathrm{ZnO}$ nanowires. To the author's knowledge this 
was the first time that a working pressure sensor had been built from $\mathrm{ZnO}$ nanowires. After growing a number of nanowires, a potential problem was identified, namely the placement of the contacts to measure the output voltage of the sensor. This problem was, however, easily overcome by making small notches in the silicon base to add wires for measurement purposes. These contacts are, however, not very robust and broke easily. Special silver paste can be used to make the contacts more robust. The special silver paste is applied and then placed in an oven at $80^{\circ} \mathrm{C}$ for a few hours. This makes the contact very strong and it should be better than the silver paste that was used in this project. Normal wirebonding techniques can also be used. The wirebonding facilities at Stellenbosch are limited and need extra preparation of a sample. The area where wirebonding is to be performed must be covered in a thick layer of gold. After the nanowires have been grown, it is difficult to add a layer of gold to one specific area. The method used was sufficient for this project.

The gluing together of two separate substrates gave the most problems. All the nanowires were measured before the gluing step. This was done in order to know the height of the supports that were necessary for the gluing. If both sets of nanowires were $200 \mathrm{~nm}$ long, the supports were made about $300 \mathrm{~nm}$ to make sure the nanowires would make contact. The main problem is that the silicon substrates are not of uniform thickness. The supplier of the substrates gives the mean thickness plus a variation. The silicon substrates used were $350 \mu \mathrm{m}$ thick with a variation of $50 \mu \mathrm{m}$. This makes it impossible to make supports for gluing of one size that will work all the time. If the nanowires are grown on a substrate that is $380 \mu \mathrm{m}$ thick and the supports are $350 \mu \mathrm{m}$ thick, the nanowires will never be in contact, because the supports will be $30 \mu \mathrm{m}$ too thick. During testing a number of devices did not work, and the gluing is the only viable explanation. The thickness of the substrate must be determined beforehand, and must be measured to within a few tens of nanometers to ensure that the device will be functional. A different method for gluing the two separate substrates must be found if the thickness of the substrates cannot be accurately determined.

The measurements were done without problems. The generated output voltages were very small, in the order of millivolts, and to ensure correct measurements an amplifier was used. The output voltage was amplified one thousand times to make sure that a signal was present, and not just noise. The 
output voltage rose with an increase in applied pressure, as expected. The output voltage also exhibited the same type of behaviour as bulk materials and related products.

Adding the amplifier removed all the advantages the nanowire based sensor had above conventional sensors, because power now had to be supplied to the amplifier. The sensor also became bulky, due to the the external power source that was required for the amplifier. In order to get a practical sensor, the output voltage must thus somehow be increased to a few volts, measurable without an amplifier. This can be done by maximising the number of active nanowires during bending, or by minimising the losses throughout the system.

The output voltage of the sensor was dependant on the area where the pressure was applied, being higher near the output wires where the silver paste had been applied. When the silver paste intruded between the two samples forming the sensor, the output voltage became higher. This is because silver has a much lower resistance than $\mathrm{ZnO}$. The silver acts as a better conductor for the output current and the system thus exhibits fewer losses. This idea was not taken any further. If both samples are covered in a thin layer of a material with low resistance, the output voltage should increase. A Schottky contact must be present in order for the piezoelectric effect to take place. One of the pair of samples is already covered in a thin layer of gold to form a Schottky contact between the two samples. The other sample could then be covered with a thin layer of gold to ensure the Schottky contact and also be a better conductor of electricity.

$\mathrm{ZnO}$ nanowires that generate voltages in the range of volts have not yet been demonstrated. Throughout the literature the output voltage remains only a couple of millivolts. There are, however, a couple of methods that can be tested to see if the voltage can be increased. In Chapter 4 a list of losses is discussed. If these losses can be minimized, the output voltage can be maximized. These losses can be controlled and altered to some degree. The simulated results show that the output of one single nanowire should be in the range of a few hundred millivolts. This is already a hundred times larger than the practical measurements that was obtained. The simulations do not take all the different impurities and losses that is present into account. This makes it clear that if those impurities and losses can be minimized the output voltage will rise considerably. Doping the nanowires is one method that can 
be investigated to try and increase the output voltage. Adding certain materials can add extra electrons or holes to the nanowires, which can increase the electron flow and thus increase the voltage. The silicon substrate is also not a good conductor, and adding a more conductive layer before the gold could also help. The only conductive layers present after nanowire growth are the bases of the nanowires, that are in contact with each other. If a good thick conductive layer is present there, the loss in electron transport will also be less.

Current measurements were also performed, but with mixed results. The instrument used to measure current is very old and had not been used for the last couple of years. The exact same measurements were performed a couple of days apart and very different results were obtained. Currents in the order of a few micro-Amperes were measured the first time around, and then on later occasions, the currents stayed at a few pico-Amperes. The second set of measurements compared well with measurements found in literature. The first set of measurements was not valid, possibly due to the instrument not working correctly. Further current measurements were thus not performed. In order to get reliable current measurements, a current amplifier will be required, so that it can be measured with conventional equipment. The current should also increase when the samples are covered with better conductive layers, as described above. The current is dependant on the output voltage as well as the resistance of the system, as described in Chapter 4. When the output voltage is increased and the system resistance is decreased, there should be a drastic rise in the output current. This is because the current and voltage are directly proportional and current and the system resistance are inversely proportional. If the system resistance is lower, as described above, the current should increase more than the voltage does. This is because the voltage will increase and the resistance decrease, having a double effect on the current.

The theory described was, however, not tested as part of this thesis. The theory will be applied and tested as part of future work at Stellenbosch University. The theory is completely new and no research has been done before on that. If the theory works and a thousandfold increase in both voltage and current can be realized, the sensor can be used as an alternative energy source.

The current, and thus the output power, needs to be raised by a sub- 
stantial amount to make these sensors useable for everyday electronics. The methods suggested above to increase the output voltage will hopefully also increase the output current to the same extent. Output power in the range of a few milli-Watts will be more than enough to power small electronic devices. The nanowires can be integrated with clothes and shoes, or any object that has constant pressure or vibration present. The devices will then generate power, due to the piezoelectric effect, which in turn can power small electronic devices.

The sensor can also be placed on the wing of a plane to continuously monitoring the pressure over the entire wing to determine where the most pressure is applied. This can help to detect weak points on the wing.

The results of this project are very promising. The concept of making a working pressure sensor from Zinc Oxide nanowires was demonstrated to be viable. There is still a lot of room for improvement, but the basic concept works. Harvesting "free energy" and using it to power devices, or to make small sensors, is possible with $\mathrm{ZnO}$ nanowires. Both the output voltage and output current must, however, be raised considerably in order for such a device to be viable. 


\section{Bibliography}

[1] [Online]. Available: http://www-03.ibm.com/ibm/history/exhibits/ vintage/vintage4506VV1003.html

[2] IBM, 2002. [Online]. Available: http://domino.research.ibm.com/ comm/research.nsf/pages/r.nanotech.timeline.html

[3] K. Hambrice, December 2004. [Online]. Available: http://www. sensorsmag.com/sensors/article/articleDetail.jsp?id=360729

[4] Z. L. Wang, “Nanopiezotronics," Advanced Materials, vol. 19, p. 889, 2007.

[5] Z. Wang, "Towards self powered nanosystems: From nanogenerators to nanopiezotronics," Advanced Functional Materials, vol. 18, p. 1, 2008.

[6] Z. L. Wang and J. Song, "Piezoelectric nanogenerators based on zinc oxide nanowire arrays," Science, vol. 312, p. 242, 2006.

[7] A. D. McNaught and A. Wilkinson, Compendium of Chemical Terminology, Oxford, Ed. Blackwell Scientific Publications, 1997.

[8] Y. Qin, X. Wang, and Z. L. Wang, "Microfibre nanowire hybrid structure for energy scavenging," Nature, vol. 451, p. 809, 2008.

[9] J. Liu, P. Fei, J. Zhou, R. Tummala, and Z. L. Wang, "Toward high output-power nanogenerator," Applied Physics Letters, vol. 92, p. 92, 2008.

[10] P. X. Gao, Y. Ding, W. Mai, W. L. Hughes, C. Lao, and Z. L. Wang, “Conversion of zinc oxide nanobelts into superlattice structured nanohelices," Science, vol. 309, p. 1700, 2005. 
[11] M. H. Huang, Y. Wu, H. Feick, N. Tran, E. Weber, and P. Yang, "Catalytic growth of zinc oxide nanowires by vapor transport," Advanced Materials, vol. 13, p. 113, 2001.

[12] 2009. [Online]. Available: http://www.whatman.com/ PRODAnoporeInorganicMembranes.aspx

[13] R. Ramaseshan, S. Sundarrajan, and R. Jose, "Nanostructured ceramics by electrospinning," Applied Physics Reviews, vol. 102, p. 102, 2007.

[14] Operation Instructions easyScan 2 STM Version 2.1.

[15] Operation Instructions easyScan 2 AFM Version 2.1.

[16] J. C. Monica, July 2009. [Online]. Available: http:/ /www.nanolawreport.com/2009/07/articles/ private-spending-on-nano-exceeds-government-spending-for-first-time/

[17] M. Berger, August 2007. [Online]. Available: http://www.nanowerk. $\mathrm{com} /$ spotlight $/$ spotid=2372.php

[18] J. Lewis, April 2009. [Online]. Available: http://www. merid.org/NDN/more.php?articleID=1860\&search=/NDN/archive. php $\% 3 F d o S e a r c h \% 3 D 1 \% 26 i t e m s \% 3 D 20 \% 26 q \% 3 D b l o o d-t e s t i n g \%$ 26sortField\%3D\%26submit\%3DSearch\&scorePrecent $=12$

[19] A. Nasir, March 2010. [Online]. Available: http://www.medgadget. com/archives/2010/03/nanotechnology_promises_a_glamorous_ future_for_cosmetics_and_skincare_products.html

[20] M. Hanlon, January 2010. [Online]. Available: http://www.gizmag. $\mathrm{com} / \mathrm{go} / 3134 /$

[21] C. Tema, November 2007. [Online]. Available: http:/ / www.dst.gov.za/media-room/press-releases / south-africa-launches-first-nanotechnology-innovation-centres

[22] [Online]. Available: http://research.ee.sun.ac.za/sand/

[23] [Online]. Available: http://crnano.org/whatis.htm

[24] nanowerk, 1 2007. [Online]. Available: http://www.nanowerk.com/ nanotechnology/introduction/introductiontonanotechnology1.html 
[25] [Online]. Available: http://www.nano.gov/html/facts/whatIsNano. html

[26] G. Elert, 1999. [Online]. Available: http://hypertextbook.com/facts/ 1999/BrianLey.shtml

[27] C. D. Klaassen, Casarett and Doull's Toxicology: The Basic Science of Poisons. McGraw-Hill Professional, 2001.

[28] T. Harper, 2003. [Online]. Available: http://www.iop.org/EJ/ abstract/0957-4484/14/1/001

[29] J. Tersoff and D. R. Hamann, "Theory of the scanning tunneling microscope," Physical Review B 31, vol. 1, pp. 805 - 813, 1985.

[30] [Online]. Available: http://www.foresight.org/nano/history.html

[31] K. A. Cook, 2005. [Online]. Available: http://www.discovernano. northwestern.edu/whatis/History/

[32] December 2008. [Online]. Available: http://www.nanowerk.com/ spotlight/spotid=8531.php

[33] M. M. A. Afghani, January 2009. [Online]. Available: http: //nanotechlaw.blogspot.com/

[34] R. Paull, December 2003. [Online]. Available: http://www.forbes. com/2003/12/29/czjw1229soapbox.html

[35] J. Wolfe, October 2006. [Online]. Available: http://www.forbes.com/ 2006/01/10/apple-nano-injw0109soapbox.inl.html

[36] B. Ehrmann and T. Kuzma, June 2005. [Online]. Available: http: //www.nnin.org/nnin_nanoproducts.html

[37] Nanoshop, 2005. [Online]. Available: http://www.nanoshop.com/

[38] D. Barnes, April 2009. [Online]. Available: http://www.modified. com/tech/0208scctirepressureguide/index.html

[39] J. F. Harvey, Theory and Design of Modern Pressure Vessels. Van Nostrand Reinhold, 1974. 
[40] [Online]. Available: http://www.sensorland.com/HowPage059.html

[41] R. W. P. King, Fundamental Electromagnetic Theory. New York: Dover, 1963.

[42] D. Lessem. [Online]. Available: http://www.ehow.com/how-does 4609318_piezoresistive-pressure-sensors-work.html

[43] M. Anissimov. [Online]. Available: http://www.wisegeek.com/ what-is-piezoelectricity.htm

[44] [Online]. Available: http://www.tech-faq.com/piezoelectric-effect. shtml

[45] M. Design, Sensor Sense: Piezoelectric Force Sensors. Penton Media, 2008.

[46] [Online]. Available: http://www.piezo.com/tech4history.html

[47] [Online]. Available: http://www.noliac.com/History_-6584.aspx

[48] C. A. Hempstead, "Piecing together the history of piezoelectricity," Metascience, vol. 18, p. 293, 2009.

[49] Z. L. Wang, "The new field of nanopiezotronics," Materials Today, vol. 10, p. 20, 2007.

[50] Z. L. Wang, X.Y.Kong, Y. Ding, P.X.Gao, W. L. Hughes, R. S. Yang, and Y. Zhang, "Semiconducting and piezoelectric oxide nanostructures induced by polar surfaces," Advanced Functional Materials, vol. 14, p. 943, 2004.

[51] Z. W. Pan, Z. R. Dai, and Z. L. Wang, "Nanobelts of semiconducting oxides," Science, vol. 291, p. 1947, 2001.

[52] X. Y. Kong and Z. L. Wang, "Spontaneous polarization induced nanohelixes, nanosprings, and nanorings of piezoelectric nanobelts," Nano Letters, vol. 3, p. 1625, 2003.

[53] X. Y. Kong, Y. Ding, R. Yang, and Z. L. Wang, "Single crystal nanorings formed by epitaxial self coiling of polar nanobelts." Science, vol. 303, p. 1348, 2004. 
[54] Q. Zhao, P. Klason, and M. Willander, "Growth of zno nanostructures by vapor liquid solid method," Applied Physics A, vol. 88, p. 27, 2007.

[55] J. Zhou, Y. Gu, P. Fei, W. Mai, Y. Gao, R. Yang, G. Bao, and Z. L. Wang, "Flexible piezotronic strain sensor," Nano Letters, vol. 8, p. 3035, 2008.

[56] M. H. Huang, S. Mao, H. Feick, H. Yan, Y. Wu, H. Kind, E. Weber, R. Russo, and P. Yang, "Room temperature ultraviolet nanowire nanolasers." Sceince, vol. 292, p. 1897, 2001.

[57] C. Soci, A. Zhang, B. Xiang, S. A. Dayeh, D. P. R. Aplin, J. Park, X. Y. Bao, Y. H. Lo, and D. Wang, "Zno nanowire uv photodetectors with high internal gain," Nano Letters, vol. 7, p. 1003, 2007.

[58] J. H. He, C. Hsin, L. Chen, and Z. Wang, "Piezoelectric gated diode of a single zno nanowire," Advanced Materials, vol. 19, p. 781, 2007.

[59] M. Law, L. E. Greene, J. C. Johnson, R. Saykally, and P. Yang, "Nanowire dye sensitized solar cells," Nature Materials, vol. 4, p. 455, 2005.

[60] B. A. Buchine, W. L. Hughes, F. L. Degertekin, and Z. L. Wang, "Bulk acoustic resonator based on piezoelectric zno belts," Nano Letters, vol. 6, p. 1155, 2006.

[61] J. Liu, P. Fei, J. Song, X. Wang, C. Lao, R. Tummala, and Z. L. Wang, "Carrier density and schottky barrier on the performance of dc nanogenerator," Nano Letters, vol. 8, p. 328, 2008.

[62] Y. Gao and Z. L. Wang, "Electrostatic potential in a bent piezoelectric nanowire. the fundamental theory of nanogenerator and nanopiezotronics," Nano Letters, vol. 7, p. 2499, 2007.

[63] [Online]. Available: http://www.efunda.com/materials/piezo/ piezo_math/background.cfm

[64] [Online]. Available: http://www.theory.caltech.edu/people/jhs/ strings/str141.html

[65] Intel, 2009. [Online]. Available: http://www.intel.com/technology/ architecture-silicon/32nm/index.htm 
[66] ScienceDaily, 12 2007. [Online]. Available: http://www.sciencedaily. com/releases/2007/12/071218105420.htm

[67] Hawk, 11 2008. [Online]. Available: http:/ /www.understandingnano. com/nanoparticles.html

[68] P. G. Collins and P. Avouris, "Nanotubes for electronics," Scientific American, p. 67, 2000.

[69] Postma, H. W. Ch., T. Teepen, Z. Yao, M. Grifoni, and C. Dekker, "Carbon nanotube single-electron transistors at room temperature," Science, vol. 293, p. 76, 2001.

[70] Eurekalert, "Beyond batteries: Storing power in a sheet of paper," Eurekalert.org, 2007.

[71] [Online]. Available: http://www.sciencedaily.com/releases/2007/ 07/070719011151.htm/

[72] MIT, "Mit on batteries."

[73] [Online]. Available: http://encyclopedia.farlex.com/Carbon-60

[74] J. M. Spero, B. Devito, and L. Theodore, Regulatory chemical handbook. CRC Press, 2000.

[75] H. B. Palache, C. and C. Frondel, Danas system of mineralogy, 1944.

[76] Y. Cui, L. J. Lauhon, M. S. Gudiksen, J. Wang, and C. M. Lieber, "Diameter-controlled synthesis of single-crystal silicon nanowires," Applied Physics Letter, vol. 78, p. 2214, 2001.

[77] B. Gu, G. M. Kim, G. T. Dusberg, G. S. Chiu, P. W. Krstic, V. Roth, and W. Han, "Growth and electrical transport of germanium nanowires," Journal of Applied Physics, vol. 90, p. 5747, 2001.

[78] R. S. Wagner and W. C. Ellis, "Vapor-liquid-solid mechanism of single crystal growth," Applied Physics Letters, vol. 4, p. 89, 1964.

[79] X. Duan, Y. Huang, Y.and Cui, J. Wang, and C. M. Lieber, "Indium phosphide nanowires as building blocks for nanoscale electronic and optoelectronic devices," Nature, vol. 409, p. 66, 2001. 
[80] Z. G. Bai, D. P. Yu, H. Z. Zhang, Y. Ding, S. Q. Gai, X. Z. Hang, Q. L. Hiong, and G. C. Feng, "Nano-scale geo2 wires synthesized by physical evaporation," Chemical Physical Review B, vol. 303, p. 311, 1999.

[81] G. Shi, C. M. Mo, W. L. Cai, and L. D. Zhang, "Photoluminescence of zno nanoparticles in alumina membrane with ordered pore arrays," Solid State Communications, vol. 115, p. 253, 2000.

[82] Z. Zhang, G. Meng, Q. Xu, Y. Hu, Q. Wu, and Z. Hu, “Aligned zno nanorods with tunable size and field emission on native si substrate achieved via simple electrodeposition," Journal of Physical Chemistry, vol. 114, p. 189, 2010.

[83] 10 2009. [Online]. Available: http://www.google.co.za/search?hl= en\&rlz=1C1CHMB_enZA326ZA328\&q=Vapor+liquid+solid\&btnG= Search\&meta $=\& a q=\mathrm{f} \&$ oq $=$

[84] X. Wang, J. Song, and Z. L. Wang, "Nanowire and nanobelt arrays of zinc oxide from synthesis to properties and to novel devices," Journal of Materials Chemistry, vol. 17, p. 711, 2007.

[85] N. L. P. Prete and L. Tapfer, "Nanostructure size evolution during aucatalysed growth by carbo-thermal evaporation of well-aligned zno nanowires on (100)si," Applied Physics A: Materials Science E Processing, vol. 88, p. 21, 2007.

[86] [Online]. Available: http://goldbook.iupac.org/H02902.html

[87] G. Kenanakis, "Growth of z-axis oriented zno nanowires from aqueous solution: the decisive role of a seed layer for controlling the wires diameter," Master's thesis, Center of Materials Technology and Laser, School of Applied Technology, Technological Educational Institute of Crete, 2001.

[88] M. Willander, L. L. Yang, A. Wadeasa, S. U. Ali, M. H. Asif, Q. X. Zhao, and O. Nur, "Zinc oxide nanowires: controlled low temperature growth and some electrochemical and optical nano-devices," Journal of material chemistry, vol. 19, p. 1006, 2009.

[89] E. Ogut, "Electroactivity of electrospun pvdf fiber mats and zno/pvdf composites," Master's thesis, Sabanci University, 2007. 
[90] J. Mohler, Electroplating and Related Processes. Chemical Publishing Co, 1969.

[91] [Online]. Available: http://nobelprize.org/educationalgames/ physics/microscopes/scanning/index.html

[92] [Online]. Available: http://physics.nist.gov/GenInt/STM/text.html

[93] [Online]. Available: www.nanosurf.com

[94] [Online]. Available: http://www.nanoscience.com/education/AFM. html

[95] D. J. Müller. [Online]. Available: http://www.mih.unibas.ch/Booklet/ Booklet96/Chapter3/Chapter3.html

[96] [Online]. Available: http://www.windaus.de/shop/index.php

[97] [Online]. Available: http://www.olympusmicro.com/

[98] [Online]. Available: http://www.headwayresearch.com/

[99] [Online]. Available: http://www.suss.com/index

[100] KERN TEE Version 1.0 11/2006.

[101] [Online]. Available: http://www.betelco.com/sb/phd/ch3/c34.html

[102] Comsol, 1 2010. [Online]. Available: http://www.comsol.com/

[103] Comosl, 1 2010. [Online]. Available: http://www.comsol.com/ company/

[104] Comsol, 1 2010. [Online]. Available: http://www.comsol.com/ products/multiphysics/

[105] I. B. Kobiakov, "Elastic, piezoelectric and dielectric properties of zno and cds single crystals in a wide range of temperatures," Solid State Communications, vol. 35, p. 305, 1980.

[106] JMP, “Design of experiments.”

[107] D. Coleman and D. C. Montgomery, "A systematic approach to planning for a designed industrial experiments," Technometrics, vol. 35, p. 1, 1993. 
[108] S. Shafiein, A. Nourbakhsh, B. Ganjipour, M. Zahedifar, and G. VAkiliNezhaad, "Diameter optimization of vls synthesized zno nanowires, using statistical design of experiment," Nanotechnology, vol. 18, p. 355708, 2007.

[109] D. C. Montgomery, Design and Analysis of Experiments. Wiley International, 2005.

[110] Stat-Ease, 8 2009. [Online]. Available: http://www.statease.com/

[111] Scopus, 2 2010. [Online]. Available: http://www.scopus.com.ez.sun. ac.za/home.url

[112] G. I. of Technology, April 2005. [Online]. Available: http://www. nanoscience.gatech.edu/zlwang/

[113] A. B. Joshi, A. E. Kalangea, D. Bodasc, and S. Gangala, "Simulations of piezoelectric pressure sensor for radial artery pulse measurement," Materials Science and Engineering B, vol. 10, p. 1016, 2010.

[114] C. Willis, October 2005. [Online]. Available: http://www. androidworld.com/prod59.htm 


\section{Appendix A}

\section{Appendix A}

Appenix A shows a number of pictures taken with the AFM, SEM and light microscopes of grown $\mathrm{ZnO}$ NWs. Different methods were used to produce the images. The caption of each picture gives a brief description of the method used and the appropriate parameters.

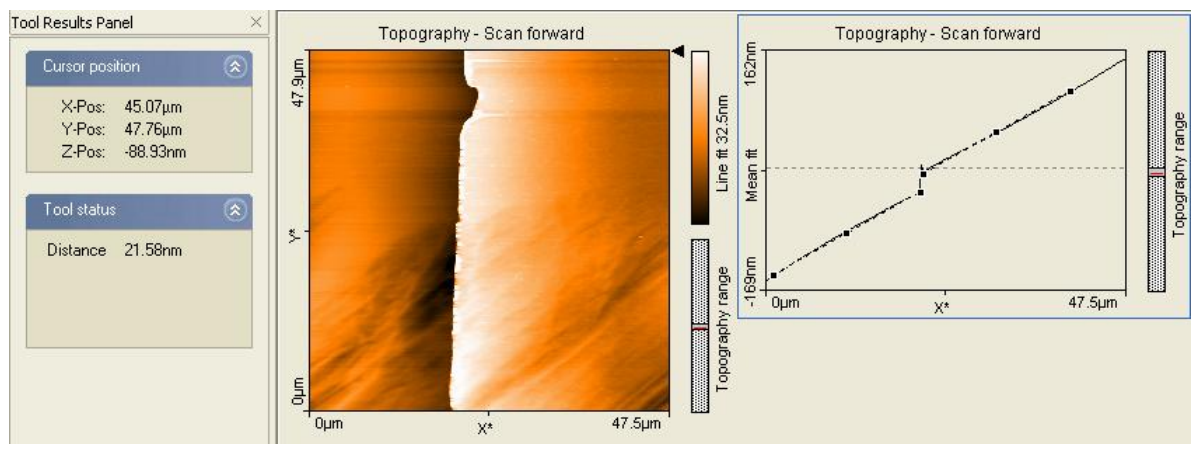

Figure A.1: AFM picture of a $20 \mathrm{~nm}$ layer of gold deposited on a Si substrate. 


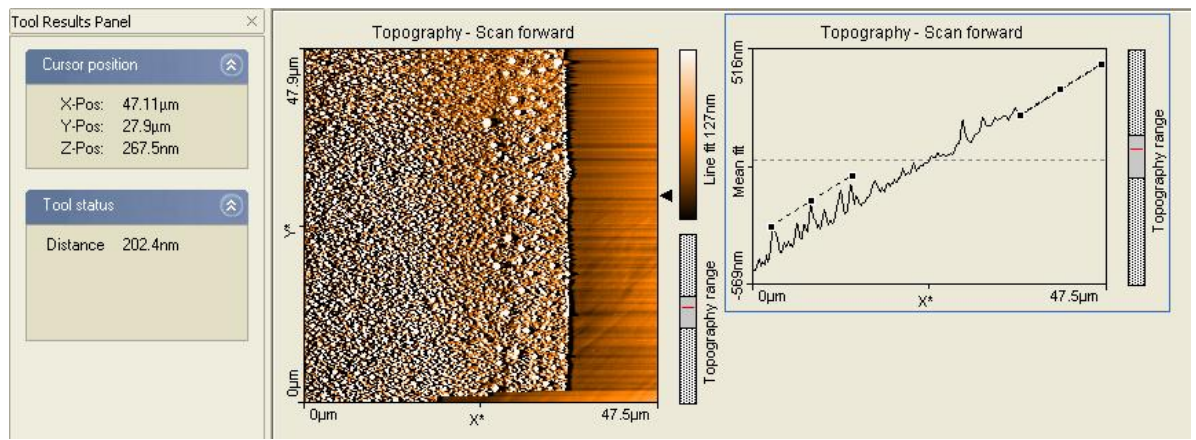

Figure A.2: AFM picture of ZnO NWs grown via VLS, with a length of about 200 $\mathrm{nm}$. The parameters for growth were as follows: Time: $30 \mathrm{~min}$, Temperature: $800^{\circ} \mathrm{C}$, Gold Layer: $25 \mathrm{~nm}$ and Ar flow rate: $80 \mathrm{sccm}$.

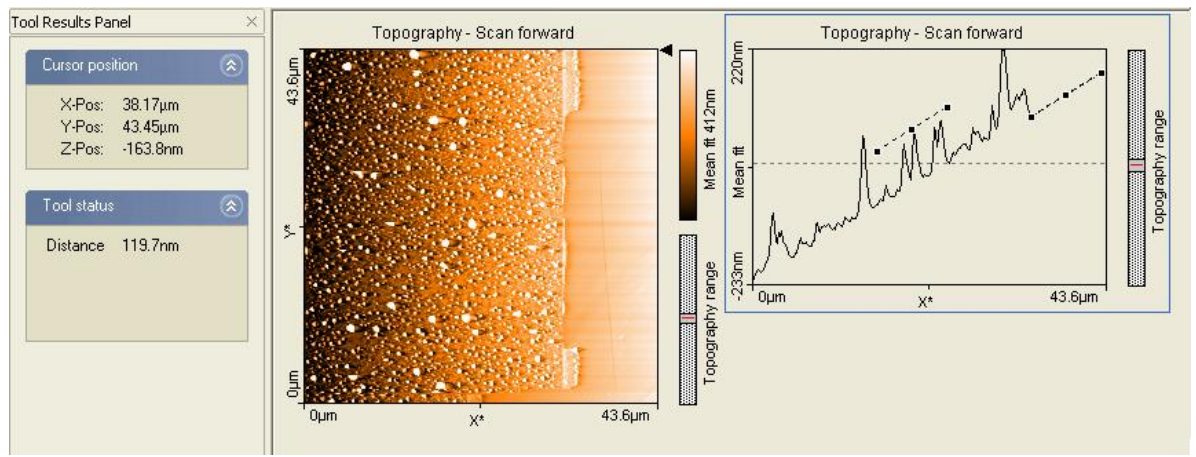

Figure A.3: AFM picture of ZnO NWs grown via VLS, with a length of about 120 $\mathrm{nm}$. The parameters for growth were as follows: Time: $40 \mathrm{~min}$, Temperature: $700^{\circ} \mathrm{C}$, Gold Layer: $20 \mathrm{~nm}$ and Ar flow rate: $120 \mathrm{sccm}$.

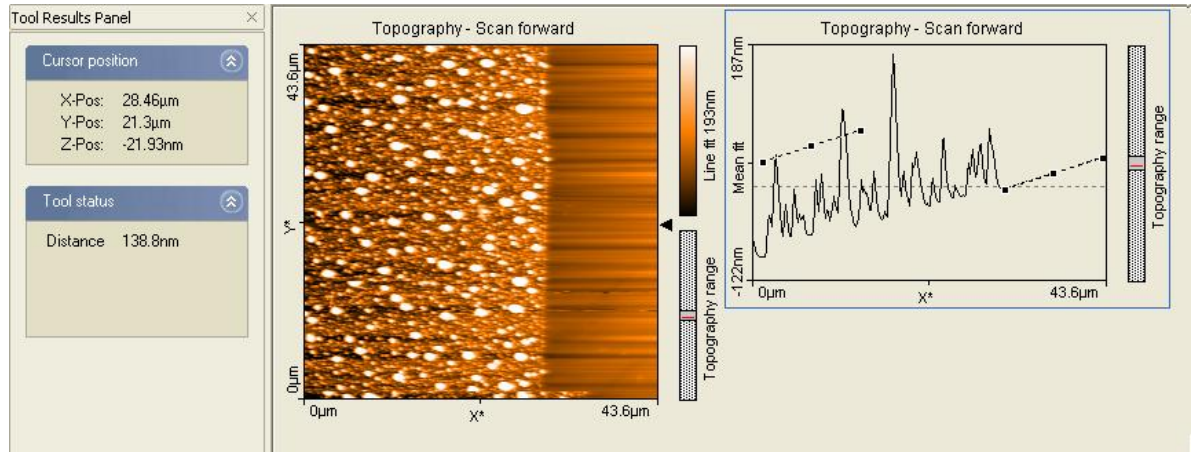

Figure A.4: AFM picture of ZnO NWs grown via VLS, with a length of about $150 \mathrm{~nm}$. The parameters for growth were as follows: Time: $10 \mathrm{~min}$, Temperature: $1000^{\circ} \mathrm{C}$, Gold Layer: $20 \mathrm{~nm}$ and Ar flow rate: $60 \mathrm{sccm}$. 


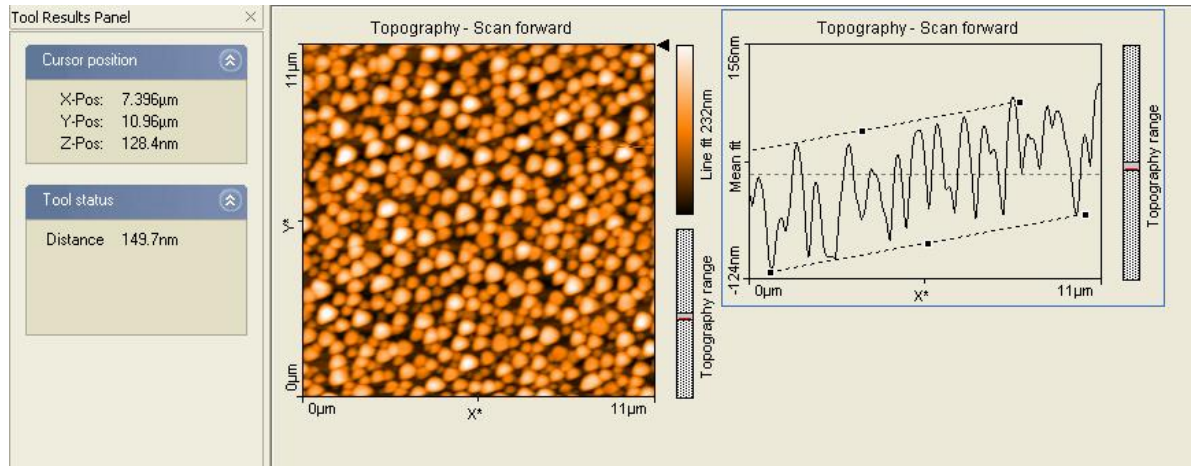

Figure A.5: AFM zoomed-in picture of $\mathrm{ZnO}$ NWs grown via VLS, with a length of about $150 \mathrm{~nm}$. The parameters for growth were as follows: Time: $10 \mathrm{~min}$, Temperature: $1000^{\circ} \mathrm{C}$, Gold Layer: $20 \mathrm{~nm}$ and Ar flow rate: $60 \mathrm{sccm}$.

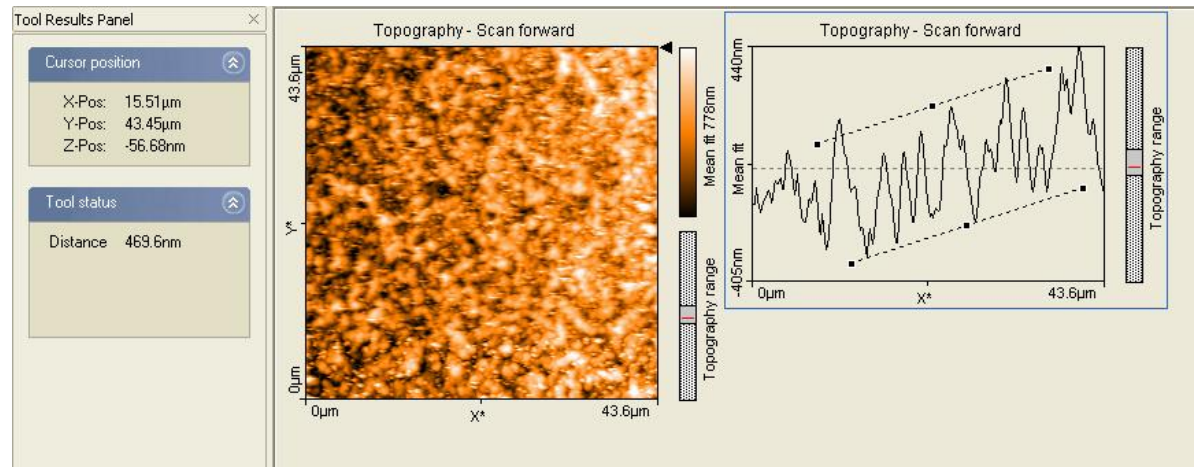

Figure A.6: AFM picture of $\mathrm{ZnO}$ NWs grown via the CVD method. The seed layer was deposited from a solution with a concentration of $0.05 \mathrm{M}$. Growth was achieved from a solution with a concentration of $0.01 \mathrm{M}$, at $95^{\circ} \mathrm{C}$ for $5 \mathrm{~h}$. 


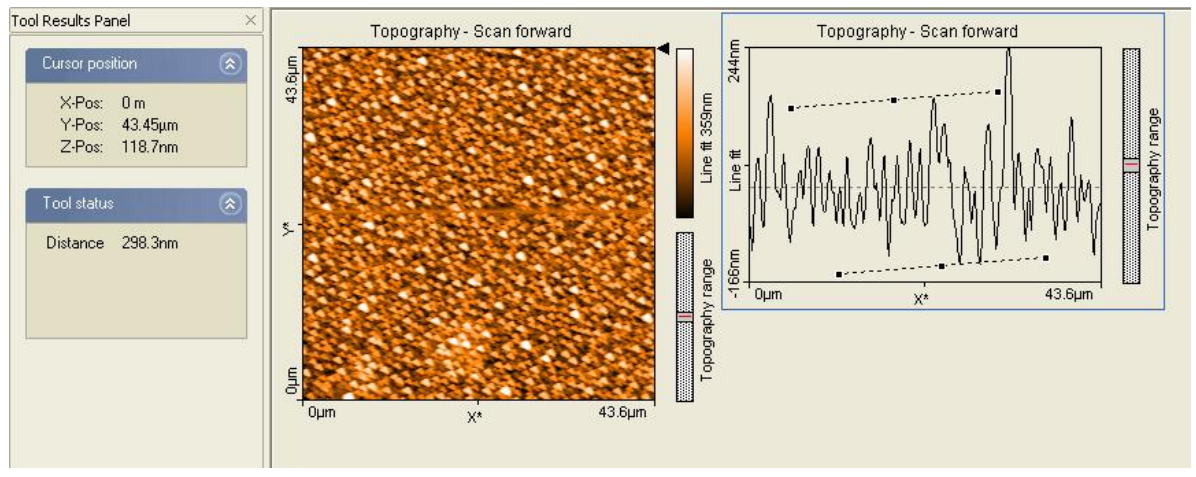

Figure A.7: AFM picture of $\mathrm{ZnO}$ NWs grown via the CVD method. The seed layer was deposited from a solution with a concentration of $0.05 \mathrm{M}$. Growth was performed from a solution with a concentration of $0.02 \mathrm{M}$, at $95^{\circ} \mathrm{C}$ for $3 \mathrm{~h}$.

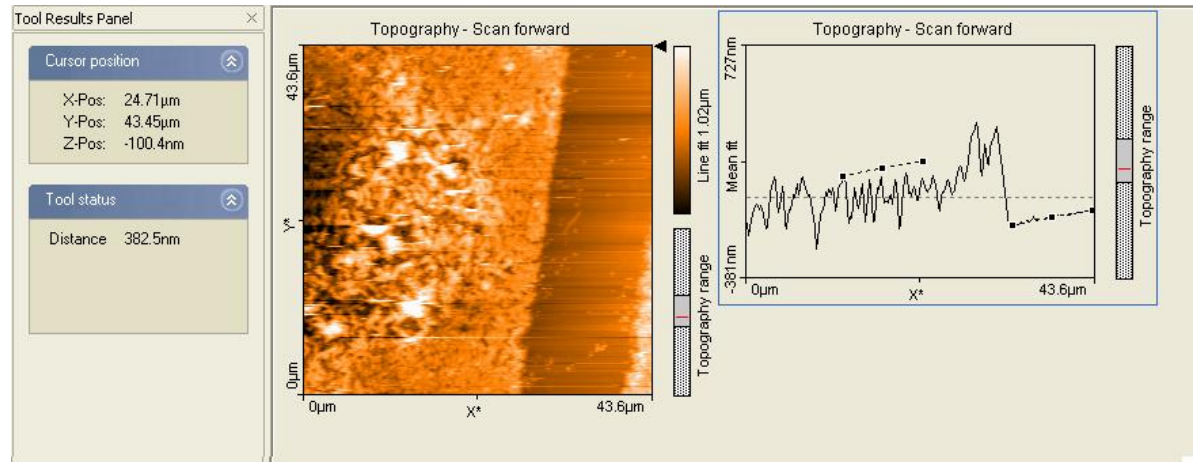

Figure A.8: AFM picture of $\mathrm{ZnO}$ NWs grown via electrodeposition, with a length of $380 \mathrm{~nm}$. The parameters for growth were as follows: Temperature: $70^{\circ} \mathrm{C}$, Time: $3 \mathrm{~h}$, Concentration: $0.1 \mathrm{M}$, Current: $0.8 \mathrm{~mA}$. 


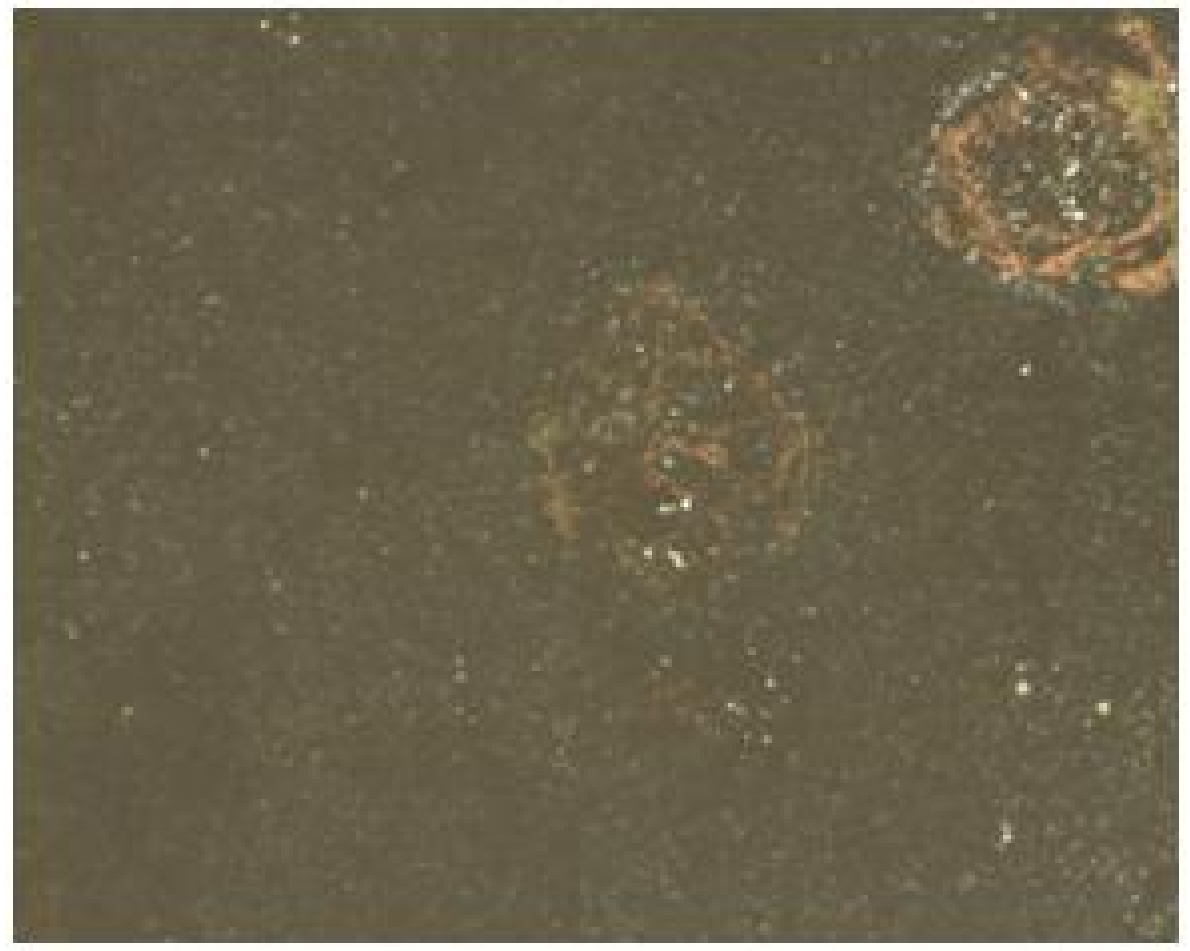

Figure A.9: Light microscope picture of Figure A.7. The dark area is a bundle of grown $\mathrm{ZnO}$ NWs. 


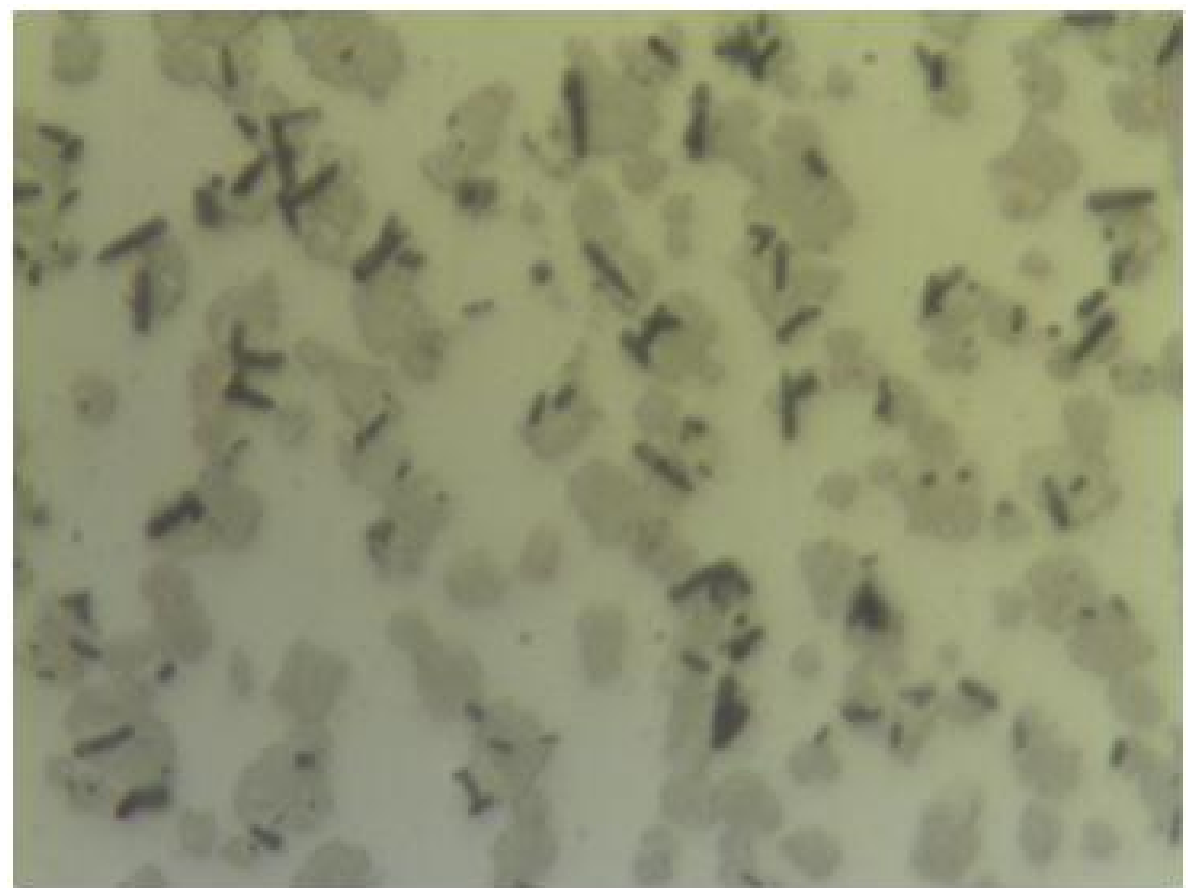

Figure A.10: Light microscope picture of $\mathrm{ZnO}$ NWs grown via CVD method. The parameters for growth were as follows: $0.01 \mathrm{M}$ concentration for growth at $95^{\circ} \mathrm{C}$ for $21 \mathrm{~h}$. The NWs did not align vertically, but rather grew in any direction. The small black rectangles in the picture are $\mathrm{ZnO}$ NWs. 


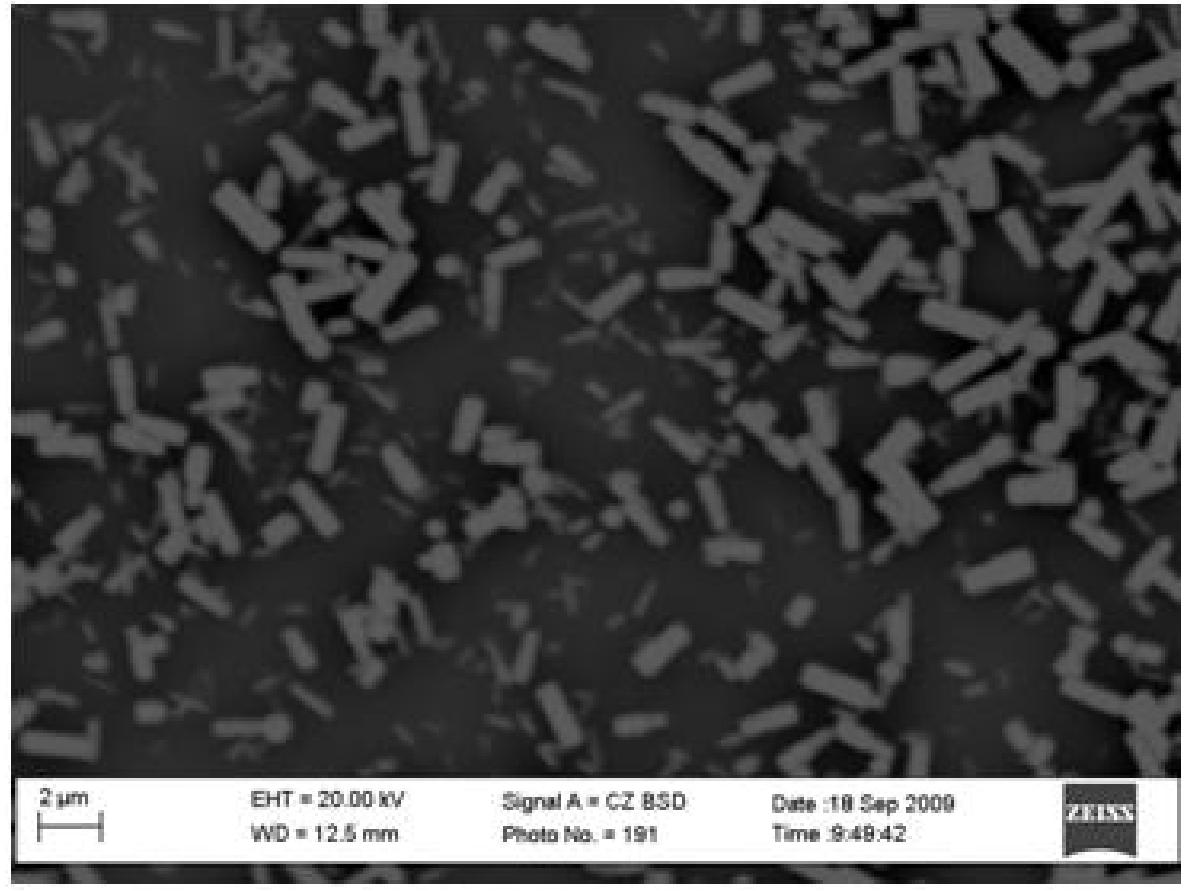

Figure A.11: SEM picture of the growth described in Figure A.10. 


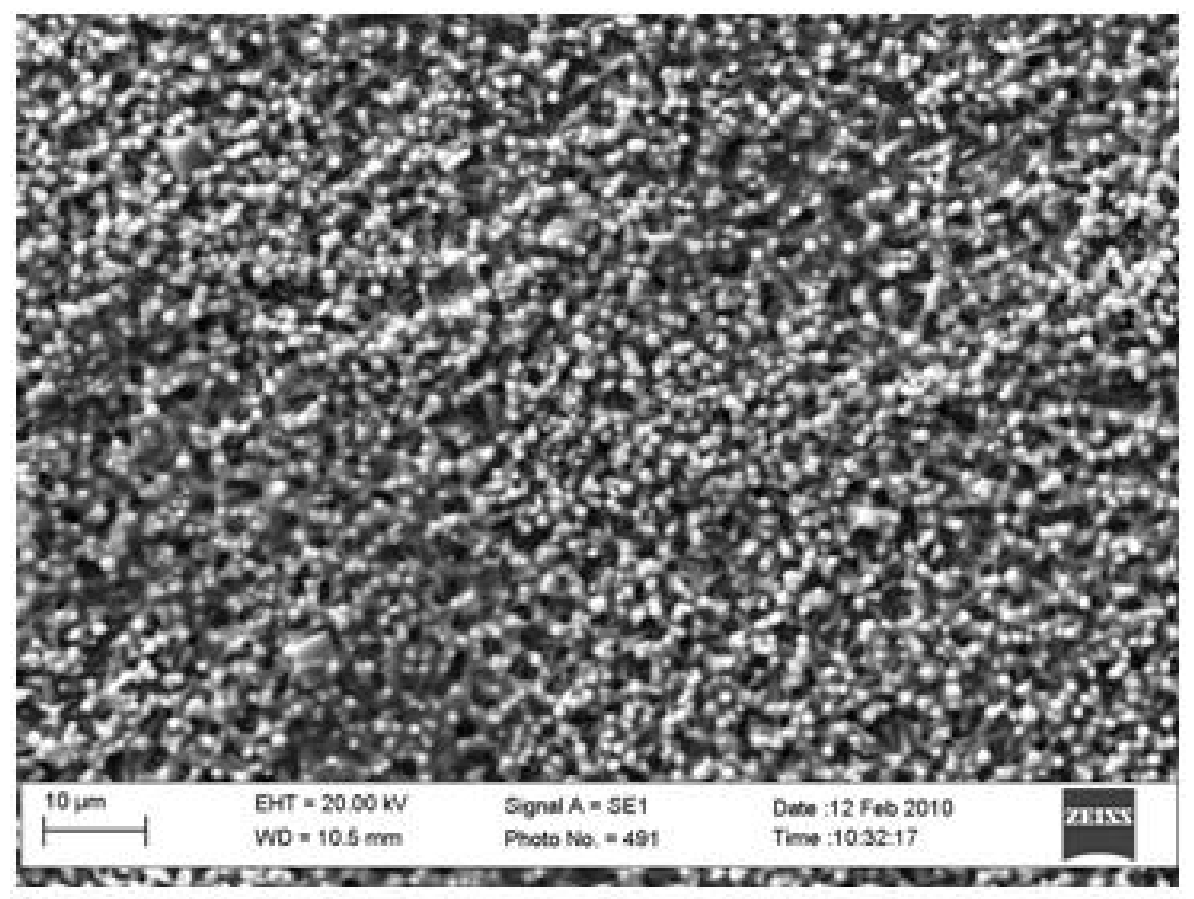

Figure A.12: SEM picture of growth described in Figure A.8. 


\section{Appendix B}

\section{Appendix B}

Appendix B shows a time schedule for the two years spent on research for the thesis. The schedule was, however, not followed exactly but did give a good indication of how long each section can take. The thesis was completed ahead of schedule. Each month was divided into four weeks and a goal was set for each week. Each week was then divided further, but this is not shown. 


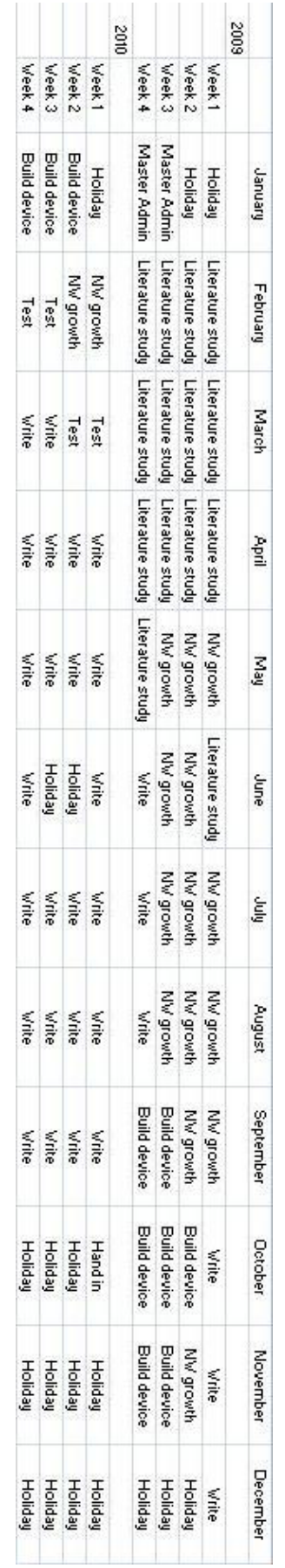

Figure B.1: Timing Schedule for 2009 and 2010 